ROADMAP

\section{Roadmap on quantum light spectroscopy}

To cite this article: Shaul Mukamel et al 2020 J. Phys. B: At. Mol. Opt. Phys. 53072002

View the article online for updates and enhancements.

\section{Recent citations}

- Detecting entanglement of unknown
$\frac{\text { continuous variable states with random }}{\text { measurements }}$
Tatiana Mihaescu et al
- A perspective on high photon flux
$\frac{\text { nonclassical light and applications in }}{\text { nonlinear optics }}$
Th. Lamprou et al
- Entanglement-Assisted Absorption
$\frac{\text { Spectroscopy }}{\text { Haowei Shi et al }}$




\title{
Roadmap
}

\section{Roadmap on quantum light spectroscopy}

\author{
Shaul Mukamel ${ }^{1,28,29} \oplus$, Matthias Freyberger ${ }^{2}$, Wolfgang Schleich ${ }^{2,3}$ (1), \\ Marco Bellini $^{4}$ (1) , Alessandro Zavatta ${ }^{4}$ (10), Gerd Leuchs ${ }^{5,6}$ (1) \\ Christine Silberhorn ${ }^{7}$, Robert W Boyd ${ }^{5,7,8}$, Luis Lorenzo Sánchez-Soto ${ }^{9}$, \\ André Stefanov $^{10}\left({ }^{(1)}\right.$, Marco Barbieri ${ }^{4,11}$ (1), Anna Paterova ${ }^{12}$, \\ Leonid Krivitsky $^{12}$, Sharon Shwartz ${ }^{13}$, Kenji Tamasaku ${ }^{14}$, \\ Konstantin Dorfman ${ }^{15}$ (D) , Frank Schlawin ${ }^{16}$, Vahid Sandoghdar ${ }^{5}$ (1), \\ Michael Raymer ${ }^{17}$ (D), Andrew Marcus ${ }^{18}$, Oleg Varnavski ${ }^{19}$, \\ Theodore Goodson III ${ }^{19}$, Zhi-Yuan Zhou ${ }^{20}$, Bao-Sen Shi ${ }^{20}{ }^{(1)}$, \\ Shahaf Asban ${ }^{1}$, Marlan Scully ${ }^{3,21,22}$, Girish Agarwal $^{3}$, Tao Peng ${ }^{3}$, \\ Alexei V Sokolov ${ }^{3,21}$, Zhe-Dong Zhang ${ }^{3}$ (1), M Suhail Zubairy ${ }^{3}$, \\ Ivan A Vartanyants ${ }^{23,24}$ (1), Elena del Valle ${ }^{25,26}$ (1) and Fabrice Laussy ${ }^{26,27}$ \\ ${ }^{1}$ University of California, Irvine, United States of America \\ ${ }^{2}$ Ulm University, Germany \\ ${ }^{3}$ Texas A\&M University, United States of America \\ ${ }^{4} \mathrm{CNR}$ - Istituto Nazionale di Ottica, Italy \\ ${ }^{5}$ Max Planck Institute for the Science of Light, Erlangen, Germany \\ ${ }^{6}$ University of Ottawa/Max Planck U Ottawa Centre, Canada \\ ${ }^{7}$ University of Paderborn, Germany \\ ${ }^{8}$ University of Rochester, United States of America \\ ${ }^{9}$ Complutense University of Madrid, Spain \\ ${ }^{10}$ University of Bern, Switzerland \\ ${ }^{11}$ Università degli Studi Roma Tre, Italy \\ ${ }^{12}$ Institute of Materials Research and Engineering (IMRE), Agency for Science Technology and Research \\ (A*STAR), 138634 Singapore \\ ${ }^{13}$ Bar Ilan University, Israel \\ ${ }^{14}$ RIKEN, SPring-8 center, Japan \\ ${ }^{15}$ State Key Laboratory of Precision Spectroscopy, East China Normal University, People's Republic of \\ China \\ ${ }^{16}$ Oxford University, United Kingdom \\ ${ }^{17}$ Department of Physics, Oregon Center for Optical, Molecular, and Quantum Science, University of \\ Oregon, United States of America \\ ${ }^{18}$ Department of Chemistry and Biochemistry, Oregon Center for Optical, Molecular, and Quantum \\ Science, University of Oregon, United States of America \\ ${ }^{19}$ University of Michigan, United States of America \\ ${ }^{20}$ University of Science and Technology of China, People's Republic of China \\ ${ }^{21}$ Baylor University, United States of America \\ ${ }^{22}$ Princeton University, United States of America \\ ${ }^{23}$ DESY, Hamburg, Germany \\ ${ }^{24}$ NRNU 'MEPhI', Moscow, Russia \\ ${ }^{25}$ Department of Theoretical Condensed Matter Physics and IFIMAC, Universidad Autónoma de Madrid, \\ Spain \\ ${ }^{26}$ University of Wolverhampton, United Kingdom \\ ${ }^{27}$ Russian Quantum Center, Russia \\ E-mail: smukamel@uci.edu
}

\footnotetext{
${ }^{28}$ Guest editor of the roadmap.

29 Author to whom any correspondence should be addressed.
} 


\section{Abstract}

Conventional spectroscopy uses classical light to detect matter properties through the variation of its response with frequencies or time delays. Quantum light opens up new avenues for spectroscopy by utilizing parameters of the quantum state of light as novel control knobs and through the variation of photon statistics by coupling to matter. This Roadmap article focuses on using quantum light as a powerful sensing and spectroscopic tool to reveal novel information about complex molecules that is not accessible by classical light. It aims at bridging the quantum optics and spectroscopy communities which normally have opposite goals: manipulating complex light states with simple matter e.g. qubits versus studying complex molecules with simple classical light, respectively. Articles cover advances in the generation and manipulation of state-of-the-art quantum light sources along with applications to sensing, spectroscopy, imaging and interferometry.

Keywords: quantum optics, spectroscopy, photon statistics

(Some figures may appear in colour only in the online journal)

\section{Contents}

1. Introduction 3

2. Quantum engineering of the radiation field 6

3. Manipulating the character and shape of ultrashort quantum light states 8

4. Continuous-variable entanglement 10

5. Harnessing entanglement from broadband energy EPPs 12

6. Quantum multiparameter estimation and sensing with photons 14

7. Infrared spectroscopy with visible light 16

8. Spectroscopy at the atomic scale by using x-ray SPDC conversion 18

9. Nonlinear spectroscopy with quantum light 20

10. Interacting a handful of quantum emitters and photons 22

11. Nonlinear optical coherent spectroscopy with EPPs 24

12. Entangled photon nonlinear spectroscopy in organic and biological molecules 26

13. Characterizing optical properties of chiral materials with twisted photonic states 29

14. Quantum diffraction imaging with entangled photons 31

16. Hanbury Brown and Twiss interferometry at XFEL sources 36

17. The true colors of quantum light 38 


\section{Introduction}

\section{Shaul Mukamel}

Department of Chemistry and Department of Physics and Astronomy, University of California, Irvine, United States of America

The term quantum spectroscopy broadly refers to spectroscopy techniques that make use of the quantum nature of light. The application of quantum states of light such as entangled photons created by parametric down conversion, has experienced tremendous progress over the last 40 years. Originally, they were employed in the investigation of the foundations of quantum physics, such as the violation of Bell's inequalities and studies of entanglement. They later emerged as basic platforms in quantum communication protocols, information processing and in the study of few-photon interactions.

This Roadmap article focuses on an entirely different emerging class of applications: using quantum light as a powerful sensing and novel spectroscopic tool to reveal information about complex matter properties that is not accessible by classical light. It aims at bridging the quantum optics and spectroscopy communities which normally do not talk to each other since they have opposite goals: manipulating complex light states with simple matter e.g. qubits versus studying complex molecules with simple classical light, respectively. Conventional nonlinear spectroscopy uses classical light to detect matter properties through the variation of its response with frequencies or time delays. Quantum light opens up new avenues for spectroscopy by utilizing parameters of the state of light as novel control knobs and through the variation of photon statistics due to the coupling to matter.

Quantum light sources. We first cover the generation of quantum light and its properties that are crucial for spectroscopic measurements. One of the striking features of quantum light is photon entanglement. This occurs between two beams of light when the quantum state of each field cannot be described in the individual parameter space of that field. Different degrees of freedom of light can become entangled. The most common types of entanglement are their orbital angular momentum (OAM) (comes in pairs), polarization, position and momentum, time and energy. Entangled-photon pairs constitute an invaluable tool in fundamental tests of quantum mechanics-most famously in the violation of Bell's inequalities or in Hong, Ou, and Mandel's photon correlation experiments. Their nonclassical bandwidth properties have long been recognized as a potential resource for 'quantum-enhanced' applications, where the quantum correlations shared between the photon pairs may offer an advantage. It is now understood that many of these applications may also be created in purely classical settings: some two-photon interference effects originally believed to be a hallmark of quantum entanglement can be simulated by post selection of signals from classical sources. This had enabled quantum-optical coherence tomography (OCT) studies with classical light. Similarly, quantum imaging can be carried out with thermal light, albeit with reduced signal-to-noise ratio. When considering applications of quantum light, it is imperative to carefully distinguish effects that stem from genuine entanglement, from the ones that arise from classical correlations when considering applications of quantum light. The back and forth transfer of entanglement between field and matter in nonlinear spectroscopy with entangled photons offers new ways for exploiting it to study many body correlation effects. Collective resonances involving noninteracting molecules can be created by entangled light. The articles that cover state-of-the-art quantum light sources include quantum engineering of the radiation field, manipulating the shape of ultrashort quantum light states, various aspects of continuous variable entanglement, and harnessing entanglement from broadband entangled photon pairs (EPPs).

Sensing. Benefiting from the high sensitivity of optical measurements at low intensities, quantum light sources allow one to explore various sensing applications. The unique spectraltemporal features of entangled pairs result in a narrow distribution of the sum of two photon frequencies while individual photons can have a broad distribution. It is thus possible to generate a pair of entangled photons with very different energies. This property grants the possibility to conveniently study visible photons, while gaining matter information in frequency range in which detection is harder (e.g. infrared, XUV). Furthermore, photon statistics may be employed as a new spectroscopic tool. The effect of the sample on higher order field correlation functions can control material information missed by ordinary detection of light intensity. More generally, quantum light offers larger parameter space that can be manipulated in applications of quantum sensing including phase imaging, quantum sensing networks, and spectrallyresolved optical phase profiles. Advancing to multiparameter sensing may open unprecedented capabilities. Current studies have established important results for relevant cases, including multi-arm interferometry, noisy interferometry and imaging. Sensing articles include quantum multiparameter estimation and sensing with photons, infrared spectroscopy with visible light (ISVL), and quantum multiparameter estimation and sensing with photons.

Spectroscopy. Nonlinear optical spectroscopy is most commonly and successfully formulated using a semiclassical approach whereby the matter degrees of freedom are treated quantum mechanically, but the radiation field is classical. Spectroscopic signals are then obtained by computing the polarization induced in the medium and expanding it perturbatively in the incoming field(s). This description is well justified in many applications, owing to the typically large intensities required to generate a nonlinear response from the optical medium, which can be reached only with lasers. Recent advances in quantum optics extend nonlinear signals down to the few-photon level where the quantum nature of the field is manifested and must be taken into account: the enhanced lightmatter coupling in cavities, the enhancement of the medium's 
nonlinearity by additional driving fields, large dipoles in highly excited Rydberg states, molecular design, or strong focusing all provide possible means to observe and control nonlinear optical processes on a fundamental quantum level. Besides possible technological applications such as all-optical transistors or photonic quantum information processing, the quantum light also offers great promise by providing novel spectroscopic tools. Parameters of the photon field wave function can serve as control knobs that supplement classical parameters such as frequencies and time delays.

The simplest signature of the quantum nature of light is the different scaling of optical signals with light intensities: classical two-photon absorption scales quadratically with the intensity and therefore requires a high intensity to be visible against lower-order linear-scaling processes. With entangled photons, such signals scale linearly. This allows one to carry out microscopy and lithography applications at much lower photon fluxes. This scaling can be rationalized as follows: entangled photons come in pairs, as they are generated simultaneously. At low light intensity, the different photon pairs are temporally well separated, and the two-photon absorption process involves two entangled photons of the same pair. The process thus behaves as a linear spectroscopy with respect to the pair. At higher intensities, it becomes statistically more plausible for the two photons to come from different pairs, which are not entangled, and the classical quadratic scaling is recovered. This enables nonlinear quantum spectroscopy of photosensitive, for example, biological, samples at low light intensities. In this case optical signals become a function of the photonic quantum state, which can be manipulated by properties of the EPPs. Apart from affecting the signal's scaling with intensity, quantum correlations also constitute an entirely new approach to shaping and controlling excitation pathways in matter in a way that cannot be achieved with shaped classical pulses. This is because between the two absorption events in entangled two-photon absorption, the light and material system are entangled. Thus, entangled photons can be used e.g. to control exciton relaxation in molecular aggregates and semiconductors.

Nonlinear optical signals and spectroscopy with quantum light is a new development. Measured signals are given by convolutions of multi-time correlation functions of the field and the matter. This approach naturally connects to the established framework of quantum optics, where field correlation functions are analyzed with nonlinear laser spectroscopy, which investigates the information content of matter correlation functions. As such, it provides a flexible platform to explore quantum light interaction with complex systems well beyond spectroscopic applications. Simultaneous time-and-frequency resolution along non-Fourier conjugate axes is a hallmark of time-energy entanglement, and its exploitation as a spectroscopic tool offers novel control knobs to manipulate the excited-state distribution and thereby enhance or suppress selected features in nonlinear spectroscopic signals. Spectroscopy articles include spectroscopy at the atomic scale by using x-ray spontaneous parametric down conversion (SPDC), nonlinear spectroscopy with quantum light, interacting a handful of molecules and photons: nonlinear optics and polaritonic states, nonlinear optical coherent spectroscopy with EPPs, and entangled photon nonlinear spectroscopy in organic and biological molecules.

Imaging. Entangled photon sources enhance phase measurements beyond the shot-noise limit and have been recently shown experimentally to enhance the performance of imaging schemes. When one photon from an entangled pair is sent through a dispersive medium, the leading-order dispersion is compensated in photon coincidence measurements - an effect called dispersion cancellation. In the field of quantum-enhanced measurements, entanglement may be employed to enhance the precision of the measurement beyond the shot noise limit. Similarly, the spatial resolution may be enhanced in quantum imaging applications, quantum-optical coherence, as well as in quantum lithographic applications. Quantum imaging with entangled light can achieve enhanced resolution, and quantum metrology can overcome the shot noise limit. One such technique, ghost imaging (GI), holds the potential to enhance real-space and frequency measurements of time evolving systems, that benefit from entanglement assisted noise reduction. The expected molecular information is attainable only by exploiting the quantum nature of light—its phase sensitivity and real space quantum correlations-for which there is no classical analog. In this technique, a high intensity beam pumped into a nonlinear crystal produces an EPP termed signal and idler. One of the beams then interacts with a mask (i.e. double slit) while the other propagates freely. The signal is obtained by a coincidence measurement involving spatially separated detectors of the two beams. If one scans the idler beam spatially in the transverse plane, the real-space image of the mask can be recorded even though this beam did not interact with the mask. This idea can be further developed by manipulation of additional degrees of freedom, e.g. scanning the time, and frequency of the photons. The photon in the molecular arm can undergo diffraction, stimulated Raman or a four-wave mixing process. Imaging contributions include characterizing optical properties of chiral materials with twisted photonic states and quantum diffraction imaging with entangled photons.

Interferometry. Spectroscopic measurements can typically be performed in two ways. Homodyne detection constitutes a measurement of the signal photons generated from a vacuum (e.g. spontaneous emission) and thus is relatively weak. Heterodyne detection is based on the interference of the weak signal photons with a much stronger local oscillator field. Interferometric pattern then yields a measurement with much higher signal-to-noise ratio. The above terminology is commonly used by the nonlinear spectroscopy community. In quantum optics, 'homodyne detection' often refers to using a local oscillator with the same frequency of the signal. In the present nomenclature this is then a particular form of heterodyne detection. This ambiguity in the definition of 
homodyne detection can be a source of confusion. In classical spectroscopy, the signal and local oscillator fields are independently generated and, thus, no correlation is expected. Utilizing interferometric tools typically used in quantum optics such as: Mach-Zehnder, Hong-Ou-Mandel, and Franson interferometers with quantum light allows one to utilize correlations between signal and local oscillator fields. In this case, rather than measuring intensity (photon number), photon statistics measurements (e.g. photon coincidence or intensity variance) allows one to fully exploit the quantum nature of the light. Examples include biphoton spectroscopy, GI, and photon counting spectroscopy. Photon counting signals depend on multipoint correlation functions of the incoming fields. Spectroscopy is classical if all fields are in a coherent state and the observable is given by normally ordered products of field amplitudes. The appearance of field correlation functions rather than products field amplitudes may also arise from stochastic classical fields or may reflect genuine quantum field effects. These should be sorted out. Glauber's celebrated hierarchical correlation formulation of the radiation field focuses on the field characterization. For spectroscopy applications, it must be extended to include the interaction with matter. Nonlinear optical signals induced by quantized light fields are expressed using time-ordered multipoint correlation functions of superoperators in the joint field plus matter phase space. These are distinct from Glauber's photon counting formalism, which uses normally ordered products of ordinary operators in the field space. Glauber's $g^{(2)}$ function of the incoming light is directly related to its ability to induce correlations in the matter. The exploitation of strong correlations in nonlinear spectroscopy with quantum light promises new routes for probing complex quantum systems. Genuine quantum effects that may not be mimicked by properly shaped classical pulses. Inspired by the detectable change in coincidence rate of entangled pairs due to interaction with matter, a new generation of nonlinear spectroscopic applications can be developed.

Novel multidimensional spectroscopy of excitons based on time-and-frequency-gated photon coincidence counting is possible. Photon statistics excitation spectroscopy, which monitors the matter response to light with different statistics, has been implemented. Schrodinger cat states nonlinear spectroscopy inspired by quantum light has been used in the data processing of pump probe measurements with classical light. Measurements that involve a sequence of interferometers combined by beam splitters as commonly used in multiport integrated photonics devices are possible. Cascading of interferometers (multiplexing) routinely used in quantum information processing for enhancing quantum properties of light can be turned into multidimensional quantum spectrometers. Scanning the position of the beam splitters in each interferometer produces time delays in a similar manner to pulse delay scanning in time-domain experiments. The crucial difference, however, is related to the fact that each time quantum light interacts with matter it changes its state. Thus, quantum correlations of light transferred to materials will entangle matter degrees of freedom in different cascades and provide a novel information not accessible by classical quantum light. Interferometry applications include the Raman quantum eraser, Hanbury Brown and Twiss (HBT) interferometry at x-ray free-electron laser (XFEL) sources, and the true colors of quantum light.

Summary. Spectroscopy with quantum light has several merits unmatched by classical light. First, the unusual time and frequency windows for homodyne, heterodyne, and fluorescence detection arising due to the quantum nature of the light generation resulting in the enhanced resolution of the signals not feasible by classical light. Second, photon counting and interferometric detection schemes constitute a class of multidimensional signals that are based on detection and manipulation of single photons and are parametrized by the emitted photons rather than by the incoming fields. Third, the quantum nature of light manifests in collective effects in many-body systems by projecting entanglement of the field into matter. This allows one to prepare and control higher excited states in molecular aggregates, access dark multiparticle states, etc. Fourth, due to the lack of nonlinear fluctuation-dissipation relations, quantum light can manifest new combinations of field and corresponding matter correlation functions not governed by semiclassical response functions such as in parametric down conversion, sum- or difference-frequency generation, two photon- induced fluorescence, etc. Finally, pulse shaping techniques that have been recently scaled down to single photon level provide a valuable tool for multidimensional measurements using delay scanning protocols not available for classical laser experiments. This could provide an additional tool for coherent control with quantum light, or the manipulation of ultracold atoms. The combination of quantum light with strong coupling to intense fields in optical cavities may result in new coherent control techniques of chemical reactions.

This Roadmap article reviews the current status of this emerging field that combines advances in the generation and manipulation of quantum light with spectroscopy applications. This field is in its infancy and will require the combination of quantum light and information technology with complex molecules and materials and broadband spectroscopy. Many advances are on the horizon.

\section{Acknowledgments}

The support the National Science Foundation (Grant No. CHE-1663822) is gratefully acknowledged. 


\section{Quantum engineering of the radiation field}

\section{Matthias Freyberger and Wolfgang P Schleich}

\section{Ulm University and Texas A\&M University}

Status. The quantum theory of radiation [1] is as old as quantum mechanics itself. Indeed, already the 'Drei-MännerArbeit' of 1925 by Max Born, Werner Heisenberg and Pascual Jordan laying the foundations of matrix mechanics contained the essential ingredients: (i) the modes of the electromagnetic field are equivalent to an ensemble of harmonic oscillators, (ii) the vector potential and the electric field play the roles of position and momentum of these oscillators, and (iii) the transition to quantized modes is achieved by substituting for these conjugate field variables operators which satisfy the familiar Bosonic commutation relations. Hence, the quantum nature of the electromagnetic field is described by quantum states of the corresponding modes.

Conceptually the most natural quantum states of a single mode of frequency $\omega$ are the number (Fock) states [2] defined by the condition that the excitation energy $E_{n}=(n+1 / 2) \hbar \omega$ of this mode is an integer multiple $n$ of Planck's fundamental energy $\hbar \omega$. The discrete quanta of excitations are called photons. Coherent as well as squeezed states [2] are superpositions of these number states with characteristic photon distributions as exemplified in figure 1 .

Since the electromagnetic field consists of infinitely many field oscillators the corresponding state is a multi-mode state. In its most elementary version it is a direct product of single-mode states. The vacuum is an example for such a product state with no excitation in any mode.

However, more interesting are states of two or more modes which are not separable and can therefore not be represented by product states. Indeed, such entangled states play a crucial role in quantum technology with applications in computing, cryptography and metrology.

How to prepare or technologically engineer [3] an arbitrary state of the radiation field? This challenging question has no general answer and has led to a vast multitude of research directions. In this short contribution we focus on only a few trends which reflect our personal view and are still connected to fundamental physics.

Current and future challenges. In 1963 Roy J Glauber showed [4] that a classical current radiates a coherent state. However, the field of a laser is fundamentally different [5] from this class of quantum states, since it undergoes phase diffusion and its photon distribution is super-Poissonian. Moreover, nonlinear processes such as second harmonic generation or the optical parametric oscillator produce [2] squeezed states.

Due to the effect of photon antibunching [2] resonance fluorescence prepares field states on the single-photon level. Indeed, once an atom, driven by a classical light field has emitted a photon, it takes some time before a second one emerges. The origin of this delay is the need for the atom to

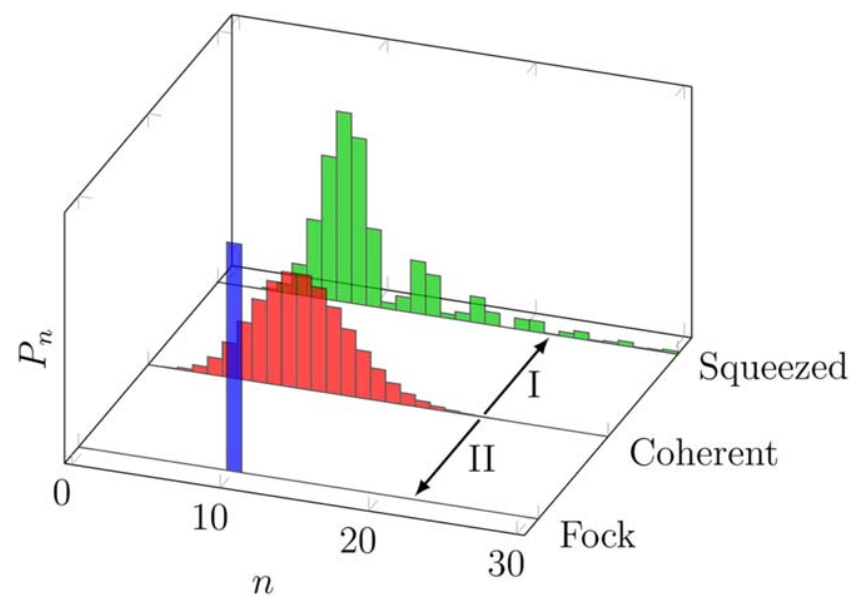

Figure 1. Two roads towards quantum state engineering: starting from the quasi-classical coherent state we can go in the direction I of a squeezed state, which is a nonclassical superposition of Fock states characterized by an oscillating photon statistics $P_{n}$. Alternatively, one can engineer in the direction II to concentrate all photons in a single Fock state.

be reexcited. Hence, in this time window there is only a single photon in the field.

Higher Fock states were first engineered in the one-atom maser [2], but today the work horse of Fock state generation relies on SPDC. This nonlinear process leads to entangled few-photon states of typically two modes. As a result, the detection of photons in one mode creates a few-photon state in the other. This important source $[6,7]$ of Fock states is a key ingredient to engineer other single and multimode field states.

Schrödinger cat states [2] are superpositions of two macroscopically distinguishable coherent states. Although the large separation in phase space between the two classical states implied by this condition makes them extremely fragile when exposed to an environment, they have been experimentally created $[8,9]$. The crucial ingredient of these methods to prepare Schrödinger cats is the entanglement between either an atom and a cavity field, or two field modes.

Although the rapid progress in experimental quantum optics has led to a zoo of readily available quantum states as summarized here, applications of quantum technology put forward numerous new demands. For example, injecting highly squeezed light into a Michelson interferometer will further increase the sensitivity to gravitational waves [10]. Moreover, entangled states such as NOON states with high photon numbers $N$ in the two modes will be very useful in novel interferometric schemes frequently used in quantum metrology. Also the just mentioned Schrödinger-cat states have been shown to enhance the signal in the novel field of quantum spectroscopy [11].

Indeed, the drive for larger squeezing, more entangled photons, higher Fock states, nonclassical superposition states as well as the search for true few-photon sources with a high efficiency are only a few of the many grand challenges of future quantum state engineering. 
Advances in science and technology to meet challenges.

Fortunately, the discovery of new quantum systems together with dramatic advances in miniaturization may overcome many of the still existing hurdles. In particular, hybrid systems, for example quantum dot micropillars and photonic circuits combining the advantages of two worlds, are extremely promising.

Other novel sources of single photons such as nitrogen vacancy centers in diamond [12] offer unique possibilities in quantum information processing and sensing. They even build a bridge into life sciences by possibly allowing us to image the brain or single cells on the quantum level.

Moreover, by incorporating glass fibres on chips complex experimental set-ups requiring valuable space can now be reduced to miniature size. This technological step opens up new avenues for commercial quantum products such as random number generators and compact quantum key distributors.

A related application of the quantum optics lab-on-thechip is the realization of boson-sampling machines [13], outlined in figure 2, which may display quantum supremacy and run faster than a classical computer. Indeed, such integrated photonic circuits with five photons have already beaten [14] early computers such as ENIAC and TRADIC.

Concluding remarks. Throughout this section we have concentrated on the preparation of a desired state of the electromagnetic field, but how to verify that we have indeed achieved our goal?

The answer to this question of a quantum state tomography consists of finding appropriate observables in combination with technological measurement devices so that the efficiently extracted information allows us to reconstruct the underlying quantum states. For example, the celebrated method of quantum state tomography [2], proposed by Karl Vogel and Hannes Risken in 1989, performs a Radon transformation of data from homodyne measurements [7]. In this way we can obtain the Wigner function [2] of the state.

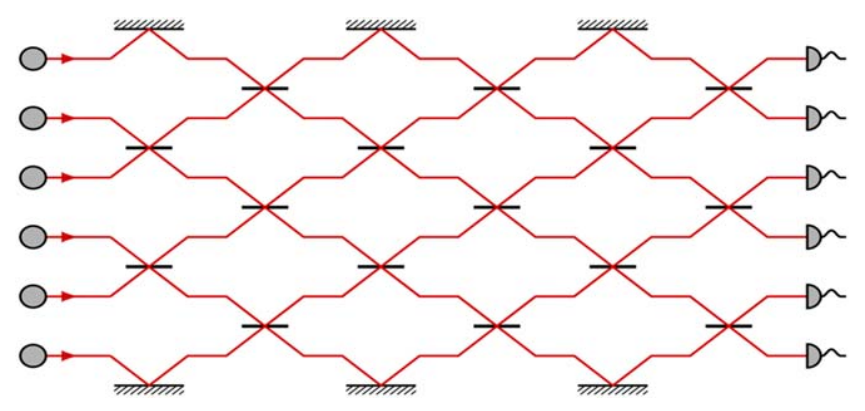

Figure 2. A photonic network of fibres connecting six single-photon sources (left) to six photon detectors (right) with the help of mirrors and beam splitters.

Today, numerous techniques to reconstruct a given state exist since any application of quantum technologies requires the complete knowledge of the prepared quantum state. Hence, both quantum state engineering and quantum state reconstruction which are obviously two sides of the same coin will remain active fields of research.

\section{Acknowledgments}

During the last decades we have profited enormously from stimulating discussions with numerous colleagues, in fact too many to list all. However, one person stands out most clearly: our close colleague Dr Karl Vogel to whom we dedicate this contribution. Sadly he passed away on 23 September, 2018. We also thank Simon Laibacher for his assistance in creating the figures. WPS is grateful to Texas A\&M University for a Faculty Fellowship at the Hagler Institute for Advanced Study at the Texas A\&M University, and to Texas A\&M AgriLife Research for the support of this work. The research of $\mathrm{IQ}^{\mathrm{ST}}$ is financially supported by the Ministry of Science, Research and Arts, Baden-Württemberg. 


\section{Manipulating the character and shape of ultrashort quantum light states}

\section{Marco Bellini and Alessandro Zavatta}

Istituto Nazionale di Ottica-CNR, Italy

Status. The possibility of exploiting the quantum nature of light in tasks where it may bring an advantage compared to its classical counterpart is at the basis of the modern race for quantum technologies. All the possible applications in the diverse fields of quantum information processing and communication, simulation, sensing, or metrology, require a precise control of the quantum states of light, from their generation to their manipulation and detection.

In the recent years, the field of quantum state engineering has quickly evolved, with new tools and techniques. Besides operations typical of the continuous-variable regime -such as squeezing-, photon addition and subtraction have demonstrated their extreme versatility for performing operations normally unavailable in the realm of Gaussian quantum optics [17]. Photon subtraction from a single-mode photonic state can de-Gaussify it and enhance its non-classicality. Moreover, it can increase and distill existing two-mode entanglement. On the other hand, photon addition has the unique capability of creating non-classicality and entanglement from scratch, whatever the input states, as shown in figure 3 [15, 18]. Sequences of photon additions and subtractions and coherent superpositions of these two operations have been used to implement novel quantum processes, emulate optical nonlinearities and test fundamental quantum rules.

However, the ability to accurately engineer quantum light states is not sufficient. It only works in the ideal situation of dealing with a single or a few, perfectly defined, modes of the electromagnetic field. Most of the times, this is just a simplification of what really goes on in a laboratory, where quantum states are often prepared in modes that do not precisely coincide with those used for their processing and detection. Moreover, operating in single modes of pre-defined shape may not be ideal, for example in the case of future quantum networks. Here, light should not only convey information through optical links, but also interact with matter systems for implementing quantum processing and memory units. These tasks and those related to the possible spectroscopic applications of quantum light demand a very specific and precise preparation of the photonic spectral and temporal mode, such that it optimally couples to different possible systems. Quantum light states with appropriate spectral and temporal shapes can also be used for enhanced time metrology [19] and for the investigation of ultrafast interactions between light and matter at the single-photon level, as shown in figure 4 [16].

For these reasons, it is of fundamental importance to gain a full control over the modes that host the quantum states. Mode engineering in the spectrotemporal domain can be achieved by properly designing the generation process. After the first realizations with atomic sources in a quasicontinuous-wave regime, the heralded production of

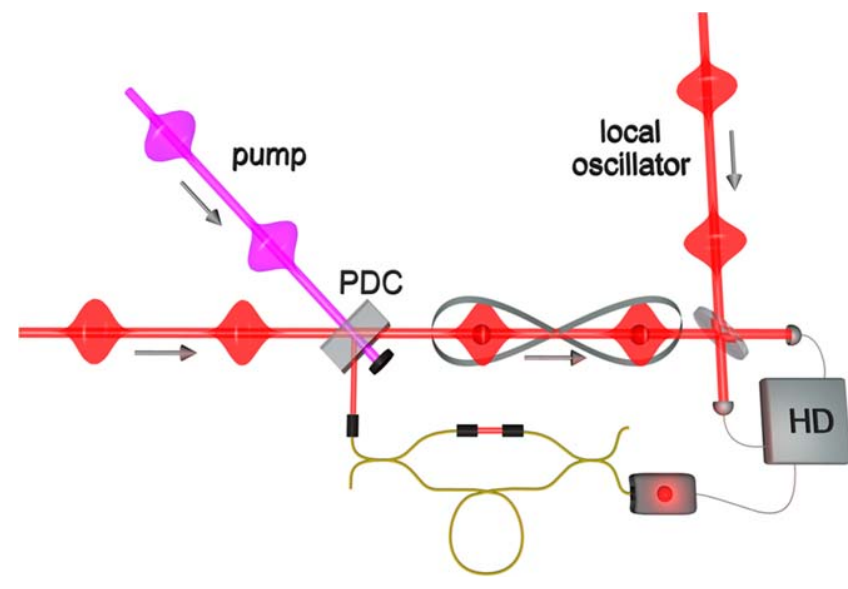

Figure 3. Scheme for entanglement generation by the conditional addition of a single photon over two consecutive temporal wavepacket modes via parametric down-conversion (PDC) [15]. Multimode homodyne tomography (HD) is performed by means of properly shaped local oscillator pulses. Reprinted by permission from Macmillan Publishers Ltd: Naure Photonics [15], Copyright 2014
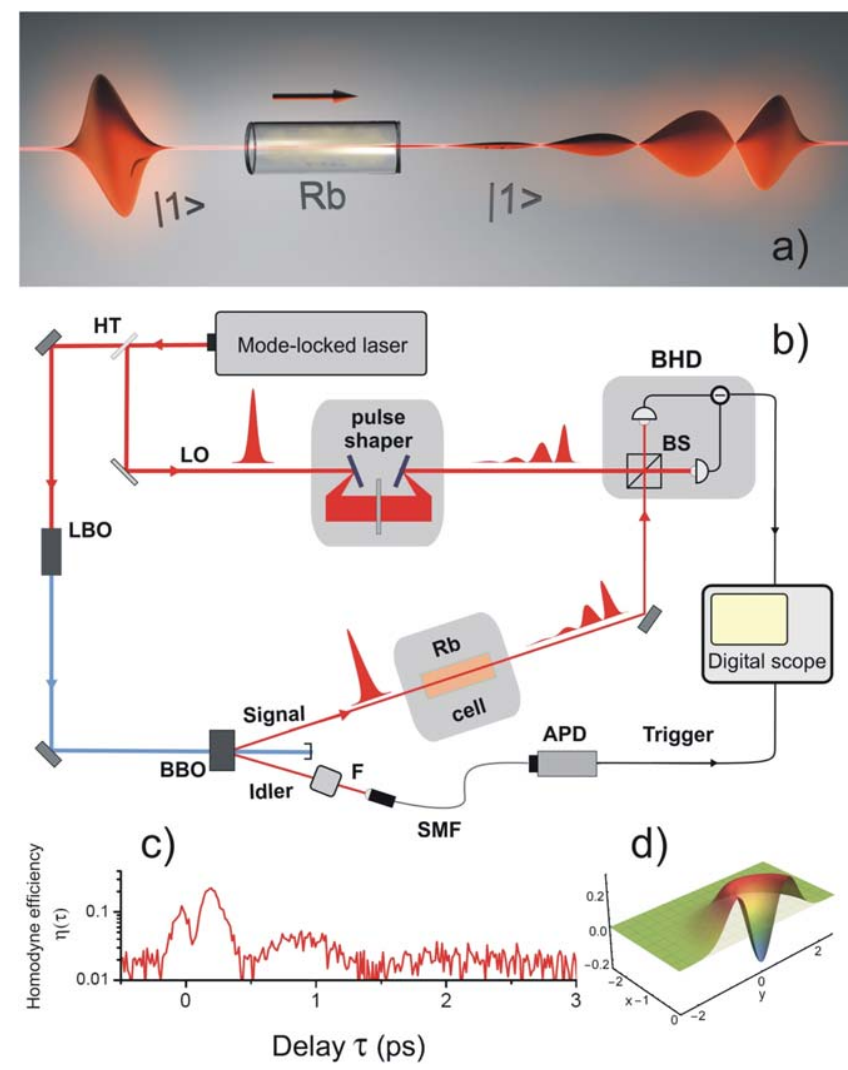

Figure 4. (a) Generation of a zero-area single photon; (b) experimental scheme for generation and homodyne detection; (c) measured time profile and (d) reconstructed Wigner function of the deformed single photon [16]. Reprinted figure with permission from [16], Copyright 2016 by the American Physical Society.

ultrashort single-photon wavepackets was obtained by adjusting the phase-matching conditions [20] or actively shaping the classical pump pulses of parametric downconversion sources [21]. Other schemes have manipulated the 
pulse mode structure of weak quantum optical states by means of nonlinear optical interaction with shaped classical control fields. Recent experiments have also made use of linear electro-optic temporal phase modulation to achieve spectral compression and shearing of single photons (see [22] for a recent review of experimental and theoretical work in the field).

Besides generating and manipulating light states with a chosen shape, one also has to develop reliable procedures to accurately characterize them, in order to make sure that the desired operations have taken place in the right modes. Recent experiments have made use of the exceptional mode selectivity of homodyne detection to probe the quantum state of light in the mode defined by reference local oscillator pulses. This ability has been used both to reconstruct the unknown spectrotemporal mode of a single photon by adaptively shaping the local oscillator, and for proving the coherent nature of the superposition of a single photon over multiple spectrotemporal modes [21]. Another promising approach has used a self-referencing method of spectral shearing interferometry to completely characterize the temporal-mode state of broadband single photons [23].

Current and future challenges. A full control over the state and mode engineering of nonclassical light is one of the major challenges in the path of future quantum technologies.

Besides the motivations introduced above, it is evident that simple single- or few-mode systems have a limited capacity of communicating, manipulating, and storing quantum information. On the contrary, gaining access to the rich mode structure of quantum light states [24, 25] might open many more opportunities, by making available several independent quantum channels on the same light beam, in much the same way as wavelength multiplexing has revolutionized the area of optical telecommunications. In particular, using the spectrotemporal shape of a light pulse as a novel degree of freedom is a powerful resource for highdimensionality quantum information processing. It has the potential of scaling up the Hilbert space spanned by multiple modes and boost the complexity of quantum networks, thus enhancing the execution of quantum information protocols in a way that is compatible with fiber transmission and waveguide integration. Accordingly, research has recently focused on developing the theoretical bases and experimental tools for time- and frequency-domain quantum information processing [26, 27]. Recent demonstrations include the realization of frequency beam splitters interfering two wavelength modes analogously to a spatial beam splitter and near-unity fidelity photonic quantum gates based on electro-optic modulation and Fourier-transform pulse shaping.

The possibility of generating nonclassical light states in arbitrary spectrotemporal modes also opens up new opportunities for spectroscopy [28]. Tailoring the photon statistics and the correlations among different modes may become a novel control knob for enhanced spectroscopy and for directly controlling and characterizing quantum dynamics of many-body states, which is not possible in classical spectroscopy.

Advances in science and technology to meet challenges. In order to face these challenges, novel theoretical and experimental tools need to be developed. From the experimental point of view, one should aim at expanding the ability to perform precise quantum operations onto arbitrary modes, as recently demonstrated for mode-tunable coherent single-photon subtraction based on sum-frequency generation [29], and delocalized single-photon addition [15]. At the same time, one should develop novel efficient techniques for manipulating spectrotemporal modes with the same versatility as can be achieved today with polarization or spatial modes. The recent promising achievements described above need to be made more reliable, compact and scalable, probably turning more and more towards integrated photonics solutions, to open new frontiers in quantum information science and for possible spectroscopic and metrological applications.

On the theoretical side, one should keep searching for novel methods of generating and manipulating non-classical and entangled states over multiple spectrotemporal modes [30]. At the same time, another hard task one should face is the development of new tools for analyzing such complex quantum states spanning multiple modes. For example, the search of efficient methods for witnessing and quantifying multi-mode entanglement is a subject of very active research. In fact, brute force approaches based on full state tomography are deemed to fail because of the complexity in the reconstruction of high-dimensional multiphoton states.

Concluding remarks. The possibility of reliably accessing the spectrotemporal mode of arbitrarily engineered quantum light states has the potential to enormously increase the range of their applications. As we have seen, by enlarging the encoding alphabet in a waveguide-compatible way, it can boost quantum information processing and communication capabilities. Equally fascinating is the possibility of using quantum light states with properly designed shapes to coherently control and steer the quantum state of matter systems.

\section{Acknowledgments}

The authors are grateful to all the co-authors of papers $[15,16,21,30]$ for fruitful and pleasant collaborations. 


\section{Continuous-variable entanglement}

\author{
G Leuchs $^{1,2,3}$, C Silberhorn $^{4}, R$ W Boyd ${ }^{1,2,5}$ \\ and $L L$ Sánchez-Soto ${ }^{1,6}$
}

${ }^{1}$ Max Planck Institute for the Science of Light

${ }^{2}$ University of Ottawa/Max Planck U Ottawa Centre

${ }^{3}$ Institute of Applied Physics, Russian Academy of

Sciences

${ }^{4}$ University of Paderborn

${ }^{5}$ University of Rochester

${ }^{6}$ Complutense University of Madrid

Status. Continuous variable entanglement appears in many different contexts and one of them is spectroscopy. The starting point is the famous gedanken experiment by Einstein, Podolski and Rosen [31], who recognized that for a pair of particles we have

$$
\left[\hat{x}_{1}-\hat{x}_{2}, \hat{p}_{1}+\hat{p}_{2}\right]=0,
$$

where $\hat{x}$ and $\hat{p}$ are the position and momentum operators of each particle. Consequently, their uncertainty product has no lower bound and the variance of both can be simultaneously zero:

$$
\Delta^{2}\left(\hat{x}_{1}-\hat{x}_{2}\right) \Delta^{2}\left(\hat{p}_{1}+\hat{p}_{2}\right) \geqslant 0 .
$$

Note that as the uncertainty in the sum (or difference) of two random variables approaches zero, the outcome of the second random variable becomes determined by the outcome of the first, since their sum (or difference) must be constant. This property leads to strong quantum correlations: $x_{1}=x_{2}$ and $p_{1}=-p_{2}$. These strong correlations, which allow for certain precise measurements, occur for any pair of conjugate operators. Examples are mechanical position and momentum, quadratures of an electromagnetic field mode, angle and angular momentum, time and frequency, or Stokes variables.

In all these cases, the state of a quantum system can be characterized by a quasi-probability distribution in the phase space spanned by the corresponding conjugate variables (figure 5). When using the Wigner function, the marginal distributions obtained by projecting onto a particular axis in phase space yields the probability distribution for the corresponding variable. The marginal distributions of two conjugate variables corresponding to orthogonal axes in phase space are related to each other by Fourier transformation. Two variables, one rotated by $\theta<\pi / 2$ with respect to the other, are related by a fractional Fourier transform. In this sense a fractional Fourier transformation can describe any rotation in phase space. Actually, the Wigner function of the EinsteinPodolsky-Rosen state, though positive definite, provides a direct evidence of the nonlocal character of this state. It is worth mentioning that, in the classical domain, the Wigner distribution is also known as the Wigner-Ville function, and it is directly related to the ambiguity function [32]. There has been little crosstalk between the fields and we expect that still much potential synergy has not yet been exploited.

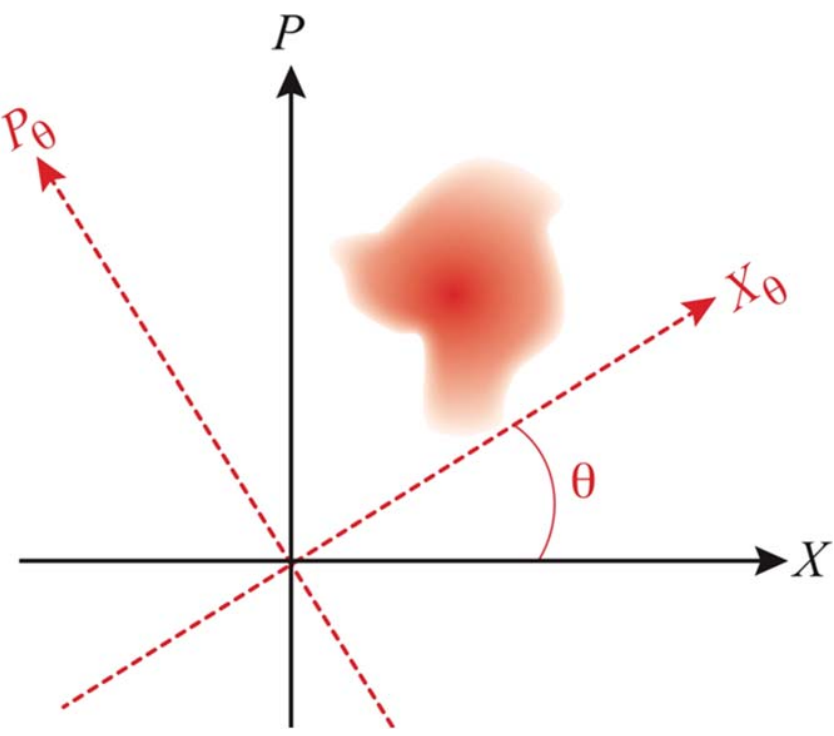

Figure 5. Two different bases $(x, p)$ and $\left(x_{\theta}, p_{\theta}\right)$ of conjugate variables spanning the phase space, one rotated by $\theta$.

The case of time and frequency is particularly intriguing because it allows for a full characterization of the spectrotemporal behavior of ultra-short pulses through self-referencing [33-35], or numerical reconstruction from spectral measurements after nonlinear interaction [36].

Current and future challenges. Generally, for two systems of the type shown in figure 5, which are entangled with each other, the problem is four dimensional, and one can generate a scenario in which $x_{1}$ and $x_{2}$ are correlated and $p_{1}$ and $p_{2}$ are anti-correlated. For field quadratures [37] such an entangled system can be generated by interfering two squeezed light modes - properly oriented in phase space - on a beam splitter [38, 39].

Entanglement in time-frequency is obtained in nondegenerate parametric down conversion [40]. The two paired photons are always created at the same time and their frequencies $\omega_{1,2}$ add up to the frequency of the pump. Frequency-time entanglement was suggested for promising applications in two-photon absorption in atoms or molecules with complex energy-level structure [41]. The energy of the final state can be selected with high precision $\Delta^{2}\left(\omega_{1}+\omega_{2}\right) \cong 0$, while at the same time the intermediate state dynamics can be measured with highest temporal resolution $\Delta^{2}\left(t_{1}-t_{2}\right) \cong 0$. The exploitation of the correlations for this type of conjugate variables can lead to an enhanced simultaneous time-frequency resolution. Moreover, it has been recently shown that time-frequency measurements based on projections onto field-orthogonal modes can be harnessed to achieve substantial improvements for resolving separations between incoherent mixtures of single-photon level signals [42].

In principle, it is possible to measure any rotated quadrature. If the $x_{1,2}$ variables are correlated and the $p_{1,2}$ variables are anticorrelated, one might wonder about the rotated $x_{\pi / 4}$ variables (see figure 5), are they not correlated? If so, how can the correlation disappear? This question was tested using polarization Stokes variables, and it was found 
[43] that the equivalents of $x_{1,2, \pi / 4}$ are indeed uncorrelated. But if one measures instead the variables $x_{1, \pi / 4}$ and $x_{1,-\pi / 4}$, one recovers the full correlation [43]. This procedure is closely related to applying one unitary operator $U$ to one subsystem and the Hermitian conjugate one $U^{*}$ to the other subsystem. States invariant under such a $U U^{*}$ operation have been called isotropic states [44] and one refers to this property as $U U^{*}$-symmetry.

Conjugate variables appear in any wave theory, classical or quantum. There is an interesting application in diffractive optics when interpreting diffraction through use of Wigner functions in phase space. The position $x$ and momentum $k$ are conjugate variables, which can be observed by direct detection, respectively, in the near and far fields. In combination with single-photon states or photon pairs, there might be an interesting application in imaging, as in [45], who succeeded in showing coincidence imaging with almost no diffraction.

Advances in science and technology to meet challenges. In the time-frequency domain the theoretical framework for the preparation of sophisticated photon-pair states with tailored continuous-variable entanglement has been established over the last years, and current experiments demonstrate an excellent control in practical systems. The design of advanced measurements techniques is still less developed, but multimode homodyne detection schemes and novel quantum measurement based on nonlinear mode-selective projections offer attractive new opportunities. In this area the exploration of new theoretical concepts for alternative measurement strategies can be expected to significantly advance the field of quantum spectroscopy. Currently, the open questions in the field are related to quantum uncertainty relationships for measurements, multiparameter estimation and entanglement projections measurements.

Concluding remarks. So far, none of the different realizations of continuous quantum variables has realized the full spectrum of quantum correlations. In particular, the application to quantum spectroscopy in the time-frequency domain is only in its infancy, and many ideas are only elaborated in theory. Nevertheless, very promising applications have already been identified. New experiments with engineered states and measurements have been started and illustrate the high potential of the field. 


\section{Harnessing entanglement from broadband energy EPPs}

\section{André Stefanov}

\section{University of Bern, Switzerland}

Status. Entanglement, a fundamental property of quantum states, enables new sensing schemes that show quantum advantage compared to classical methods. In the optical domain, entanglement can take place between the various degrees of freedom (modes) describing quantum states of light: polarization, spatial or spectral modes, and photon number. For spectroscopy and, more generally, for any quantum scheme involving spectro-temporal variables, entanglement in the energy domain has to be considered. All classical spectroscopic schemes are constructed out of a combination of various classical light sources with only few varying parameters: e.g. wavelength, polarization, pulse duration, pulse coherence. On the other hand, and thanks to entanglement, the space of parameters describing quantum light is far larger. Controlling energy-EPPs allows to access part of this space and is the most promising way toward a practical implementation of quantum spectroscopy concepts.

Currently, the most common and efficient way to generate entangled states of light is the process of SPDC in a crystal with second order nonlinearity: a photon of welldefined energy (and momentum) from a pump laser is downconverted into two photons. Owing to conservation of energy (and momentum), the emitted photons pairs can be entangled in energy (or momentum).

The first application of energy entangled (also sometime referred as energy-time entangled) photons was two-photon interferometry, demonstrating entanglement and quantum non-locality [46]. Since, energy entanglement has been applied for the encoding of high dimensional quantum states [47, 48], as well as for optical sensing, including OCT [49], dispersion [50] or temporal [51, 52] measurements. Energy entangled photons possess the quantum property to show simultaneously narrowband spectral characteristics and short temporal features. They are therefore natural candidates for the implementation of two-photon quantum spectroscopy.

The states emitted by SPDC are fully determined by the phase matching in the crystal and the pump laser properties. In the perspective of quantum spectroscopy, it is important to be able to generate and manipulate energy-EPPs fulfilling the following requirements: a high flux of photon pairs, strong entanglement, tuneability of the pump wavelength and the ability to shape the two-photon wavefunction.

Figure 6 shows the components of a potential implementation of two photon quantum spectroscopy that includes a source of photon, a flexible setup for spectro-temporal manipulation and the interaction with a probe [53].

One of the consequences of observing two-photon interaction between photon pairs and matter is the linear dependency of the absorption as a function of the photon flux, in opposite to a quadratic dependency in the case of classical coherent light. Despite the advances in the generation and

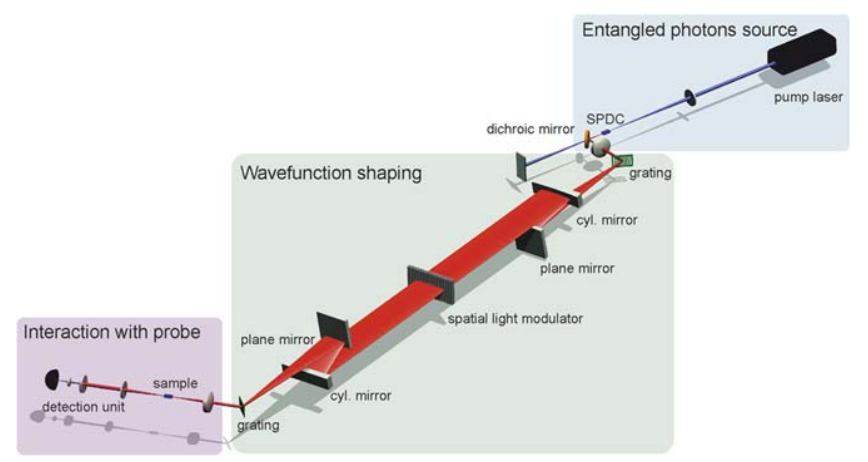

Figure 6. Scheme of a setup for the implementation of two photon quantum spectroscopy. Energy entangled photons are generated by SPDC in a nonlinear crystal. Their wavefunction is manipulated by a pulse shaper comprising dispersive elements and a spatial light modulator. The shaped photon pairs are then focused onto a sample where a two-photon process takes place.

control of entangled photons, such absorption of photon pairs was only observed in nonlinear crystals [54] and atomic systems [55]. The usually very weak two-photon crosssection of molecules precludes up to now the observation of entangled two-photon absorption in molecules for a practical realization of quantum spectroscopy.

Current and future challenges. In order to understand why it is difficult to observe entangled two-photon absorption in molecules, a comparison with the classical process helps. Classically, intense ultra-short laser pulses compensate for the weakness of two-photon interactions. Their peak intensity is in principle not limited. On the contrary, the flux of EPPs is fundamentally restricted by the condition to show pairwise entanglement. Pictorially, the photon pair stream could be represented as a train of pulses whose temporal durations are given by the photons coherence time, and that contain exactly two photons per pulse. Therefore, each energy mode should ideally not be populated by more than one pair. This limit corresponds to the bandwidth of the entangled photons. If we restrict ourselves to this case, where the photon pairs do not overlap temporally, the photon flux can thus only be improved by decreasing the coherence time, or equivalently increasing the bandwidth.

A high flux generation in SPDC requires intense pumping in order to reach the previously defined limit. In practice, the pump intensity can only be increased up to a certain point, depending on both the availability of lasers and the damage threshold of the crystals. Therefore, the efficiency of the sources has to be increased. Finally, the central wavelength of the photons, given by the pump wavelength, should be tunable, setting an additional constrain on the sources.

A broadband emission of SPDC is not only favorable in term of photon flux but also in term of the entanglement content of the state. The entanglement of the pairs is quantified by the ratio of the emitted photons spectral bandwidth to the pump bandwidth. For spectroscopy schemes that rely on entanglement, higher entanglement corresponds to better energy and temporal resolutions. 
Next to the sources, two-photon spectroscopy and coherent control with EPPs require the ability to manipulate the two-photon spectral wave-function. One of the most flexible approach is to apply pulse-shaping techniques from ultrafast laser science. Frequency shapers based on spatial dispersion of frequencies and spatial shaping have shown to applicable to entangled photons [56]. Their flexibility allows for instance to control the degree of quantumness of energy correlations [57]. The overall transmission of the setup is however much more critical as compared to classical applications, because single photon losses act quadratically on photon pairs. In addition, the energy resolution should be high enough to match the requirements for spectroscopy. Because of their linearity, such shapers cannot realize arbitrary general transformations of the quantum state and are thus limiting the range of quantum spectroscopy schemes.

Finally, and certainly the most critical challenge toward experimental implementation of quantum spectroscopy is the weakness of two-photon interactions. Even the most efficient photon pair sources would not produce a significant signal, when focused onto molecules in free-space. It is therefore crucial to find new ways to increase the interaction.

From a conceptual perspective, the distinction is not always clear between spectroscopy schemes that genuinely rely on entanglement, and schemes for which only classical correlations provide an advantage [58]. In addition, the quantum properties of light can be of relevance not only through entanglement but also through the non-classical statistics of light.

Advances in science and technology to meet challenges. In order to address those challenges, advances both from the experimental and theoretical sides have to be developed. For the generation of entangled states, engineering the phase matching condition by periodical polling in nonlinear crystal allows for very broad emission, while the efficiency can be improved by integrating a waveguide in the crystal. In addition, the use of aperiodically poled crystals allows one to create ultra-broadband EPPs [59]. However, because the phase matching in periodically polled crystals is very sensitive to the pump wavelength, tuning is only possible over a very limited range. Thus, wide range tuning requires either to switch between crystals, or crystals with fan-out poling structure. Alternately, usual thin bulk non-poled crystals have broad phase matching, with the price of much lower efficiencies. The phase matching could be tuned over a much larger range, but the pump intensity should be drastically increased, for instance by placing the crystal in an optical cavity for the pump. The next generation of SPDC sources should combine those various techniques into one flexible and efficient source.

From the perspective of shaping the two-photon wavefunction, achieving general nonlinear transformation, requires, in addition to linear shaping, changes of frequencies at the single photon level. It could for instance potentially be realized, by optical modulation, or by optical nonlinear effects.

Towards quantum spectroscopy it is needed to develop new ways to increase the interaction strength should be explored. A promising path is to confine both the entangled photons and the probes into nanostructures; thus taking advantages of field enhancement either in nanoparticles or waveguides.

Finally, a general framework of quantum spectroscopy in the context of quantum metrology, based on quantum information theoretical concepts would be of great interest for the design of new practical schemes. Schemes that do not strictly rely on photon pairs but more generally on quantum light should be developed. For instance by going beyond the photon pair regime and allow for higher photon flux [60].

Concluding remarks. Experimentally, quantum spectroscopy is more than a straightforward extension of classical spectroscopy with quantum light replacing classical lasers. All the experimental components from the source to the signal detection have to be reconsidered. New experimental techniques have still to be developed toward a realization of quantum spectroscopy that provides a practical advantages compared to present classical techniques.

Acknowledgments. This research was partially supported by the Swiss National Science Foundation through the grant PP00P2_159259. 


\section{Quantum multiparameter estimation and sensing with photons}

\section{Marco Barbieri}

Università degli Studi Roma Tre, Italy and Istituto Nazionale di Ottica-CNR, Italy

Status. The quest for quantum-enhanced measurement started when the use of quantum light was suggested as a way to improve the sensitivity of gravitational wave detectors [61]. Building on the continuous effort this proposal has brought about, quantum metrology is now established at the forefront of quantum technologies [62], with its promise to deliver optical and atomic sensors with improved capabilities. When a single parameter carries all the relevant information about the system to be probed, quantum metrology offers a clear route towards the optimal measurement: the quantum Cramér-Rao bound, that links the achievable precision on the parameter with the amount of Fisher Information available in the probe and effectively extractable though measurement. For the same number of resources (atoms, photons, etc), quantum probes carry higher Fisher Information than possible with only classical means.

The emphasis on single-parameter cases puts an unnecessary limitation to the class of problems that can be understood. On the other hand, important examples intrinsically fall in the multiparameter domain: examples include phase imaging (see figure 7), quantum sensing networks, and spectrally-resolved optical phase profiles. Furthermore, performing the simultaneous estimation of multiple parameters at once is demanded by the dynamics of the sensing process: if dissipation occurs, this will be described by its own characteristic parameters, that a simple pre-calibration cannot address.

The transition to the multiparameter regime is by no means a direct extension of the single-parameter case, but demands for a deep rethinking of the conceptual and technological methods to approach the problem. The distinctive feature is that distinct parameters are associated to transformations, which in quantum formalism may not commute [63]. This reflects onto the compatibility of the optimal measurements for individual parameters, a feature that the quantum Cramér-Rao approach captures only in part [64]. Additional considerations, for instance the saturation of the Holevo bound or the possibility to perform collective measurements, need to be considered to determine general limits [65].

Estimating multiple optical phases simultaneously in a multi-arm interferometer epitomizes the multiparameter problem, setting phase imaging within the framework of quantum metrology [66]. Optimal precision can be achieved for each individual phase, according to the quantum CramérRao bound. Non-trivial features are revealed such as the enhancement of the overall precision when interrogating all phases at once with respect to addressing them separately; however, the approach is unable to derive an explicit form of the optimal measurement, thus ad hoc inspection should be

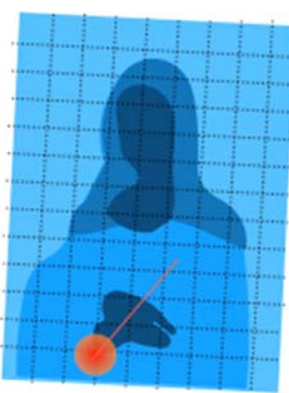

\section{single-parameter estimation}

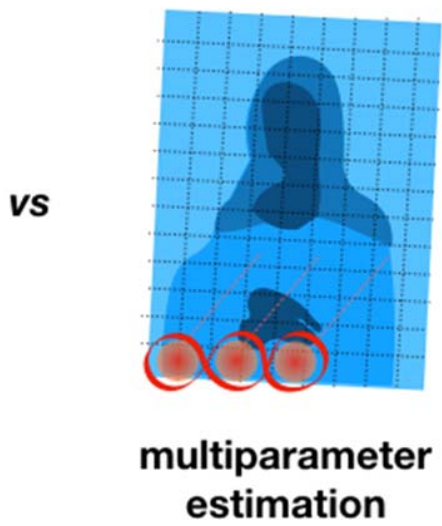

Figure 7. Single-parameter versus multiparameter estimation applied to phase imaging. The object is divided into pixels: the full image is reconstructed by assessing the optical phase imposed by each pixel. The standard strategy for quantum-enhanced phase estimation targets each pixel individually with quantum light [62]. Multiparameter estimation, instead, addresses multiple pixels, hence multiple phases, at once by distributing quantum light across the whole object.

performed. A recent experimental demonstration has shown the capabilities of the current technology at the proof-ofprinciple level [67].

Imaging is a different example for which multiparameter estimation is relevant [68]. The simplest instance considers centroid position and separation of two incoherent sources; the advantage of using quantum light has been experimentally demonstrated by means of two-photon measurement that achieves optimal precision for the centroid, and beats Rayleigh's limit for the separation [69]. The key ability resides in performing a collective measurement on the two photons at once to obtain a precision advantage on both parameters.

Similar issues are found in the simultaneous estimation of a phase in the presence of noise, reducing the interference contrast [70]. Indeed the phase is ultimately retrieved by looking at the intensity of the signal with respect to the maximum of the interference fringes. An erroneous retrieval of the phase may occur, if the maximum is not correctly know at the time when the measurement is performed. Taking the multiparameter approach to estimate the contrast while measuring the phase allows to ensure the retrieval of an unbiased phase and to maintain a quantum advantage, with a modest cost of resources, as demonstrated in a proof-ofconcept experiment with photon pairs [71]. We observe that, in principle, phase and noise can be jointly estimated at the optimal precision, it turns out that in most practical scenarios there exists indeed a trade-off, which becomes less severe as the complexity probe state or of the detection scheme increases [70].

Current and future challenges. These are three examples of the applications quantum multiparameter estimation finds. In all cases, quantum Fisher Information has provided some insights, but it falls short from providing a complete picture, as it would for single parameters. Research has sought 
clarifications in pursuing several case studies; the lack of a unifying picture is a critical point, which needs being addressed, also attempting alternative strategies to the usual Fisher information approach [72].

Few experiments have been realized so far investigating multiparameter scenarios; in particular, no investigations have addressed phase imaging. The main difficulty so far is represented by the production rate of multiphoton states, which then imposes long acquisition times at the expenses of the stability of the measurement setup; integrated optics offers viable solutions in this sense, with the possibility of realizing complex stable interferometers [73] (see figure 8). A successful implementation of quantum-enhanced spatial phase imaging would trigger a similar interest in the timefrequency domain, with multiple phases being associated to different optical frequencies, leading to dispersion measurements.

\section{Advances in science and technology to meet challenges.}

Technical limitations, slowing the transition past the proof-ofprinciple stage, mostly concern photon sources and detectors. Significant process has been accomplished over the last decade, however the most promising solutions require materials at cryogenic temperatures-semiconductor quantum dots for the sources, superconductors for the detectors.

This makes it unlikely that the first real-life applications will be delivered by widespread, table-top commercial systems. Laboratory-size facilities, working as service units operated by trained scientific personnel for the investigation of light-sensitive samples, can be foreseen. What is required to get to that stage is not much the availability of solutions to specific technical problems, but a rather general vision of what sensing systems should be like.

The development of genuinely user-friendly devices might be linked to the employ of a different approach: rather than operating at fixed photon number, continuous-variable quadratures of the optical fields can be controlled instead.

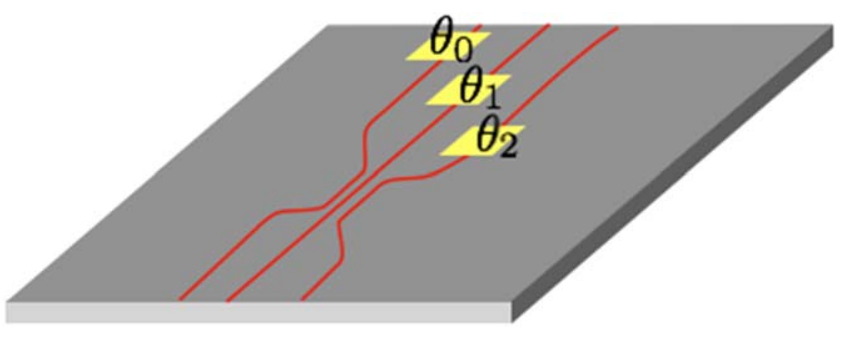

Figure 8. Integrated optical solutions offer a compact platform for implementations of multiphase estimation. The phase actuators are connected the external environment to be probes (e.g. by inserting biosamples though microfluidic channels). The use of multiparameter estimation is relevant when phase gradients need being measured.

These pose less severe requirements for their generation, based on nonlinear parametric processes, and their detection, realized by means of balanced homodyne detection. Exploration of quantum advantages in multiparameter estimation is, however, at a very early stage.

Concluding remarks. The single-parameter paradigm underlying the concept of quantum sensors is often an artificial limitation imposed to the analysis complex process. Advancing beyond this step to multiparameter sensing may open unprecedented capabilities. Current studies have established important result for relevant cases, including multi-arm interferometry, noisy interferometry and imaging. However, it may be necessary to rethink how we approach quantum estimation in order to obtain a satisfactory general picture.

\section{Acknowledgments}

We acknowledge discussions with $\mathrm{P}$ Mataloni, V Cimini, A Datta, F Sciarrino, M Genoni, I Gianani, P Humphreys, M Paris, L Pezzè, E Roccia, M Sbroscia, and M D Vidrighin. 


\section{Infrared spectroscopy with visible light}

\section{Anna Paterova, and Leonid Krivitsky}

Institute of Materials Research and Engineering (IMRE), Agency for Science Technology and Research (A*STAR), 138634 Singapore

Status. Light at infrared (IR) frequencies provides valuable information about material composition and propagates through the media with minimal scattering losses. This is why IR metrology is a practical tool for many applications in material analysis, sensing and imaging. Over the last three decades, several IR metrology techniques have been developed. Examples include Fourier transform IR (FTIR) spectroscopy, OCT, and others [74]. A significant and common limitation of existing techniques is associated with the limited performance of IR optical components and materials. Light sources, photodetectors, and imaging devices for IR light are less efficient, bulkier and costlier than devices operating in the visible range. The need of measuring material properties in IR range and the limitations of the optical instruments motivates the search for alternative approaches in IR metrology.

In the last few years, we have been developing a set of techniques, which allow retrieving the information about IR properties of the materials using light sources and detectors operating in the visible range. The approach is based on the effect of the nonlinear interference of correlated photons produced via SPDC $[75,76]$. The original concept (referred to as 'induced coherence') was introduced by L Mandel and coworkers some time ago, and it recently drew attention in the context of practical applications, such as imaging [77], spectroscopy [78-80], OCT [81] and polarimetry [82].

In SPDC, a photon from the pump laser decays in a nonlinear crystal into a pair of highly correlated photons. One photon of the pair is generated in the visible range and another photon in the IR range. Two identical nonlinear crystals pumped by the same laser form the nonlinear interferometer. Visible and IR photons generated in both crystals are superimposed, and the interference of visible photons is detected. The remarkable feature of this interferometer is that the visibility and the phase of the interference pattern for visible photons depend on amplitudes and phases of all the interacting photons: the pump, the visible and the IR. When the sample under study is inserted in the path of IR photons, the interference pattern observed for the visible photons changes. The sample properties in the IR range can then be inferred from the modified pattern. Thus the method circumvents the problem of generation and detection of IR photons.

We applied the above method to IR spectroscopy [78-81], IR OCT [82], and IR polarimetry [83]. In the IR spectroscopy experiments, we simultaneously and independently measured both the refractive index and the absorption coefficient of various samples. In IR OCT imaging, we measured the reflectivity of the hidden layers and performed $3 \mathrm{D}$ raster imaging through opaque (in the visible range)

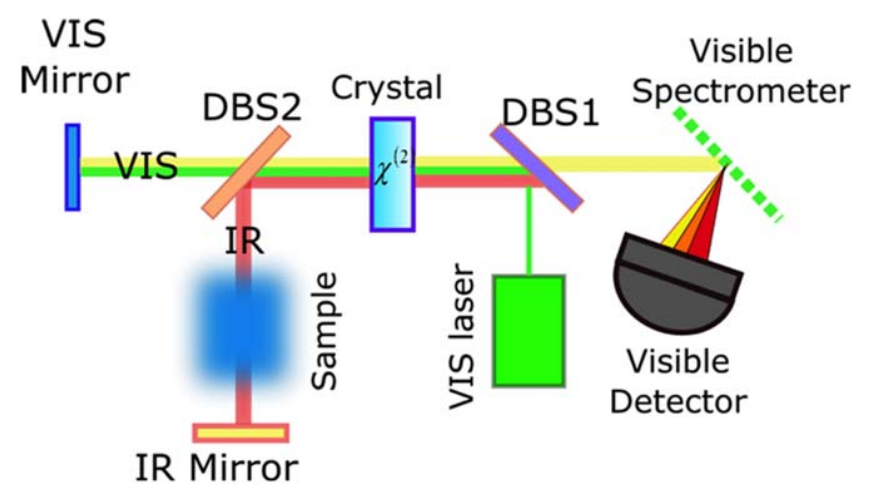

Figure 9. The scheme of the nonlinear Michelson interferometer used for ISVL. Visible and IR photons are generated in a crystal pumped the laser. The visible and IR photons are split on the dichroic beamsplitter (DBS) and then reflected into the crystal by respective mirrors. The sample is inserted in the path of IR photons. The interference pattern for visible photons is detected using the visible range spectrometer and the detector. The pattern depends on properties of the sample, which interacts with the IR photon.

\begin{tabular}{|c|c|c|c|}
\hline Technique & FTIR spectroscopy & $\begin{array}{c}\text { Direct IR } \\
\text { spectroscopy }\end{array}$ & $\begin{array}{c}\text { IR spectroscopy with visible } \\
\text { light (ISVL) }\end{array}$ \\
\hline Principle of operation & $\begin{array}{c}\text { Interference of } \\
\text { broadband IR light }\end{array}$ & $\begin{array}{c}\text { Direct light } \\
\text { absorption }\end{array}$ & $\begin{array}{c}\text { Nonlinear interference of } \\
\text { down-converted light }\end{array}$ \\
\hline Spectral range & $\begin{array}{c}\text { Broad } \\
(1.6-30 \mu \mathrm{m})\end{array}$ & $\begin{array}{c}\text { Moderate } \\
(0.5-3.5 \mu \mathrm{m})\end{array}$ & $\begin{array}{c}\text { Moderate } \\
(0.7 \sim 5 \mu \mathrm{m})\end{array}$ \\
\hline Spectral resolution & $\begin{array}{c}\text { Very High } \\
(\sim 0.5 \mathrm{~nm})\end{array}$ & $\begin{array}{c}\text { High } \\
(\sim 4 \mathrm{~nm})\end{array}$ & $\begin{array}{c}\text { Moderate } \\
(\sim 18 \mathrm{~nm})\end{array}$ \\
\hline Measurement time & Fast & Moderate & Moderate \\
\hline $\begin{array}{c}\text { Direct measurement of } \\
\text { absorption/ refractive } \\
\text { index }\end{array}$ & YES/ NO & YES / NO & YES / YES \\
\hline $\begin{array}{c}\text { Requirement for IR } \\
\text { components }\end{array}$ & YES & YES & NO \\
\hline
\end{tabular}

Figure 10. Benchmarking of IR spectroscopy techniques. We cite representative values for conventional techniques and values for initial demonstrations of ISVL [79-81].

materials. In the IR polarimetry, we measured the birefringence of various samples. The method was tested with carbon dioxide gas, silicon wafers, polymers, and other materials. These results pave the way for further adoption of this technique in practical applications. The following discussion is focused on technical developments necessary to achieve this goal.

Current and future challenges. Let us discuss key features of the proposed technique, referred here as ISVL, and benchmark them against conventional methods. The comparison of key performance parameters is shown in figure 10 .

Spectral range. The spectral range of conventional methods is defined by the bandwidth of the light source (thermal source, tunable laser etc). In contrast, the ISVL uses a single monochromatic pump laser with a fixed frequency. The SPDC process is intrinsically broadband, and its tunability is limited by the phase matching conditions and the transparency range of the SPDC crystal. Lithium Niobate is 
a convenient choice for ISVL as it provides high SPDC efficiency and the transparency range up to $5 \mu \mathrm{m}$. Though useful for many applications, this range does not cover an important fingerprint region of 8-11 $\mu \mathrm{m}$. Hence, we emphasize the importance of the development of highly efficient nonlinear optical materials transparent in a broad range of IR frequencies.

Spectral resolution. In the ISVL the spectral resolution in the IR range is defined by the spectral selection performed over visible photons. This is one of the attractive features of the method as high-performance, compact and inexpensive visible range spectrometers are readily available. However, there is another approach which allows eliminating the need for the visible range spectrometer. The method uses a narrowband SPDC source and makes use of intrinsic correlations between the SPDC frequency and spatial modes. Since the SPDC emission angles and frequencies are interrelated, the spectral information can be obtained directly from the SPDC spatial distribution. The latter can be seamlessly detected using a wide-field imaging device (e.g. CCD camera) and then processed by a specialized algorithm $[78,80]$. We believe that the implementation of such a method would greatly enhance the readout rate without the loss of the spectral resolution.

Measurement time. In the ISVL the absorption spectrum is obtained from a consecutive scan of the SPDC central wavelength, which is tuned either by changing the temperature of the crystal and/or by changing the crystal orientation. In contrast, measurement time in the FTIR spectrometer is much faster due to parallel readout of the spectroscopic signal across the full bandwidth of the light source. The spectrum is inferred from interference fringes recorded by translating the mirror in the interferometer. We anticipate that a similar approach can be realized with the ISVL taking into account recent progress in the development of efficient broadband SPDC sources.
Functionality. By default, conventional IR spectroscopic techniques measure only the imaginary part of the refractive index, associated with the absorption. Measurements of the real part of the refractive index, associated with the refraction, are more challenging. One approach relies on numerical simulations and inference of the refractive index from the absorption data. However, the method is model dependent and may not yield accurate results, especially for complicated absorption spectra. Another approach is to hook the IR spectrometer to the IR refractometer or ellipsometer. These devices directly measure the refractive index, but they are bulky and rather expensive. In contrast, the ISVL allows independent and simultaneous measurements of both real and imaginary parts of the refractive index. The former is inferred from the shift of the interference fringes and the latter from the fringes contrast. We believe that it is of interest to apply the ISVL for characterization of complex media, such as nanostructures and new materials.

Concluding remarks. We described the novel IR spectroscopy technique based on the nonlinear interference of frequency correlated photons. It overcomes some limitations of conventional IR techniques and requires only a simple setup with accessible visible-range detectors and lasers. This approach allows direct measurement of the refractive index and the absorption coefficient of a medium in the broadband IR range by detecting photons in the visible range. We outline current challenges in its practical adoption, along with some interesting application ideas.

\section{Acknowledgments}

We thank D Kalashnikov, Ch. An and S Kulik for their contribution to this project. We acknowledge the financial assistance of the Quantum Technology for Engineering program (QTE) of A*STAR. 


\section{Spectroscopy at the atomic scale by using $x$-ray SPDC conversion}

\author{
Sharon Shwartz ${ }^{1}$ and Kenji Tamasaku \\ ${ }^{1}$ Bar Ilan University, Israel \\ ${ }^{2}$ RIKEN, Japan
}

Introduction. Nonlinear interactions between x-rays and long wavelengths (infrared/visible/ultraviolet) can be utilized as an atomic scale probe for valence electron charge density and related phenomena in solids including light-matter interactions on the microscopic level [84]. The high resolution is the consequence of the atomic scale wavelengths that correspond to x-rays and the long wavelengths provide the wavelength selectivity and enhance the interaction with the valence electrons. Hence, effects that are based on X-ray nonlinear interactions can lead to a power method that combines spectroscopic and structural data for the improving of the insight on microscopic phenomena in solids.

SPDC of $\mathrm{x}$-rays to long wavelengths is a nonlinear process in which, like SPDC in the optical range, a pump beam interacts with the vacuum field to generate two correlated beams, but here one beam is at X-ray wavelengths and the wavelengths of the second beam can range between infrared wavelengths to x-rays and it is selected by phase matching. Since the wavelengths of the x-ray beams that participate in the process are on the order of the distance between the periodic atomic planes, the reciprocal lattice vector is used for phase matching. The implication of this phase matching scheme is that the intensity of the phased matched signal is proportional to the Fourier coefficient of the nonlinear current density corresponding to the selected reciprocal lattice vector. This Fourier component can be related to the Fourier component of the linear susceptibility and to the induced charge [84]. By measuring the Fourier components for different atomic planes it is possible to reconstruct the optical response of the valence electrons at the atomic scale resolution.

The idea to utilize nonlinear wave mixing effects between x-rays and long wavelengths as an atomic scale probe for valence electrons was suggested in the early 1970s [84]. Freud and Danino observed SPDC of x-ray into the soft $\mathrm{X}$-ray regime by using an $\mathrm{x}$-ray tube source in 1981 [85]. However, the progress in the field has been accelerated by the emergence of the modern high brightness synchrotron radiations sources about 25 years later. In a series of papers, Tamasaku and his colleagues demonstrated the ability to extract atomic scale information from the measurements of SPDC of x-rays into the ultraviolet (UV) regime [84-88]. They also pointed out a new physical mechanism of interference of the down converted photons with Compton scattering. More recently Schori et al measured SPDC of $\mathrm{X}$-rays to visible wavelengths [89]. In addition, high-energy resolution experiments with an $\mathrm{x}$-ray tube was reported recently [90], where in that paper the authors used modern $\mathrm{x}$-ray optics to significantly improve the flux and the signal-

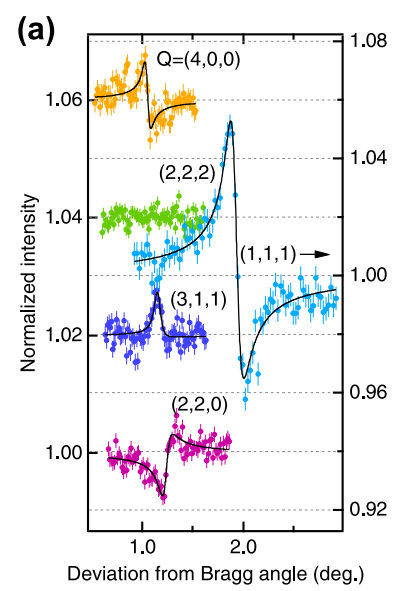

(b)

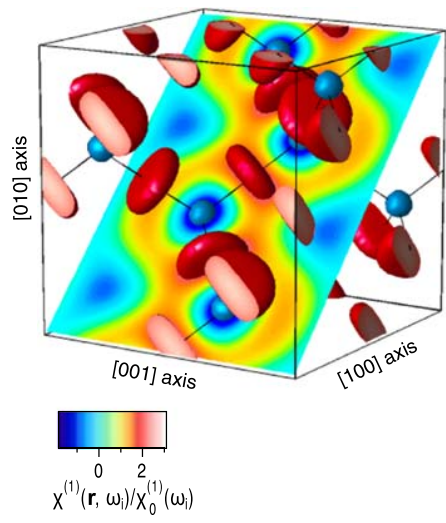

Figure 11. SPDC of $x$-rays into the extreme ultraviolet by diamonds. (a) Normalized count rate of the $\mathrm{x}$-ray signal photon as a function of the deviation angle from the Bragg angle. Labels indicate the reciprocal lattice vector used for phase matching. (b) The reconstructed linear susceptibility at the idler frequency. The cube indicates the unit cell. The red disk and the blue sphere represent the optical response by the bond and the core charge.

to-noise ratio with respect to the first demonstration in the 1980s.

$X$-ray SPDC into the extreme ultraviolet regime. Since the phase matching condition is satisfied by the reciprocal lattice vector, SPDC is observed as nonlinear diffraction. The rocking curve, which is the signal counts as a function of the pump glancing angle, represents the phase mismatch dependence of SPDC. Figure 11(a) shows the rocking curve of SPDC in diamonds measured for several reciprocal lattice vectors, $\mathbf{Q}$. The photon energy of the pump and the idler are $11.107 \mathrm{keV}$ and $60 \mathrm{eV}$, respectively.

The rocking curve shows an asymmetric peak at the phase matching angle due to the Fano interference with the Compton scattering. Analysis of the Fano effect gives the Qth Fourier coefficient of the nonlinear susceptibility. As mentioned above, the Fourier coefficient of the nonlinear susceptibility relates to that of the linear susceptibility, and, thus, one can reconstruct the real space structure of the linear susceptibility at the idler frequency by the Fourier synthesis (figure 11(b)). We note that only the amplitude of the Fourier coefficient is determined experimentally, and the phase problem should be solved separately.

The spatial resolution of the reconstructed linear susceptibility is determined by the shortest lattice spacing used for the nonlinear diffraction. As a result, the optical response at the idler frequency can be investigated with much finer resolution than the diffraction limit, i.e. half the wavelength. In case of figure 11(b), the spatial resolution of $0.54 \AA$ corresponds to only $1 / 380$ of the idler wavelength of $206 \AA$, revealing the microscopic optical response of the bond and the core charge, which is smeared out in the linear process at the wavelength.

$X$-ray SPDC into the visible regime. As we discuss in the pervious paragraph, several demonstrations of PDC into 
extreme ultraviolet were reported, but the extension of this method into the optical regime is more challenging due to the proximity of the photon energy to the input photon energy. The observation of this effect requires a very high level of collimation and high-energy resolution optics, which are currently available only at a few beamlines. The first observation of the effect was in diamond. Figure 12(a) shows the first measurement of the spectrum of the effect. The peak on the left corresponds to the elastic Bragg scattering and the broad peak is the PDC signal. The peak is observed at $7.1 \mathrm{eV}$, where the efficiency of the PDC is the largest. This energy is near the bandgap of the diamond crystal, where the density of states of the valence electrons is the highest. This observation demonstrates the ability to measure the valence electron spectral dependencies in the optical range. To demonstrate the ability to reconstruct the Fourier component of the nonlinear susceptibility from the measurement the rocking curve of $\mathrm{x}$-ray signal of PDC at an idler energy of $2.2 \mathrm{eV}$ was measured, as shown in figure 12(b).

We note that since the visible photons can be measured, it is possible to use this effect as a source for extremely nondegenerate entangled photons that can be used for the investigation of quantum effects in a new regime combining properties of $\mathrm{x}$-rays and visible radiation. However, thus far the visible photons have not been measured due to the strong visible fluorescence. This challenge might be overcome by using faster detectors or by using higher quality crystals.

Concluding remarks. The progress in nonlinear effects with $\mathrm{X}$-ray including SPDC has been accelerated in recent years. The physics of SPDC of x-rays into long wavelengths has revealed new interesting physics and holds a great promise for applications for spectroscopy at the atomic scale. The main advantages are the ability to combine structural and spectroscopic information in a single measurement and the extremely broad spectral range that can be explored with the same apparatus. However, the development of applications
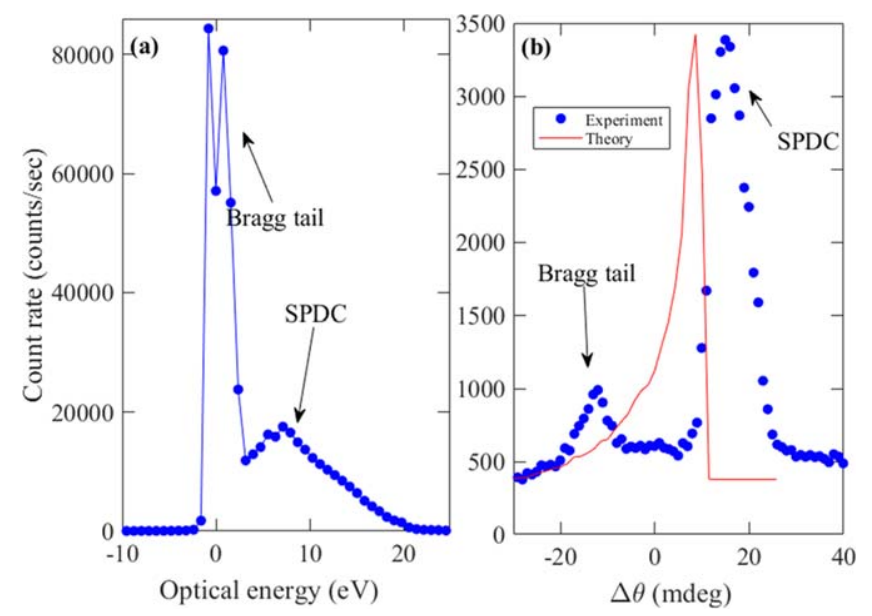

Figure 12. (a) Spectrum of the SPDC signal. The left sharp peak corresponds to the elastic Bragg scattering and the broad peak is the SPDC signal. (b) X-ray signal count rate as a function of the pump deviation angle from the phase matching angle. The small peak on the left is the residual elastic, and the peak centered at $15 \mathrm{mdeg}$ from the origin is the PDC signal. The idler central wavelength was $550 \mathrm{~nm}(\sim 2.2 \mathrm{eV})$. The blue dots are the experimental results and the solid red line is calculated from theory.

that are based on those effects still retains major challenges such as signal-to-noise ratio and low efficiencies of the effects. We anticipate significant progress with the next generation of x-ray sources, which will deliver higher flux and at better beam quality. It is also likely that the new capabilities with ultrashort $\mathrm{x}$-ray pulses will open the possibility for the use of x-ray SPDC as probe for ultrafast dynamics in materials by combining it with pump-probe techniques. Finally, we note that the by measuring the long wavelength photons it would be also possible to utilize the advantages that quantum light can offer such as the enhancement in the signal-to-noise, the resolution, and the sensitivity of the measurements as has been demonstrated with optical radiation. 


\section{Nonlinear spectroscopy with quantum light}

\author{
Konstantin E Dorfman ${ }^{1}$, Frank Schlawin ${ }^{2}$, Shaul Mukamel ${ }^{3}$ \\ ${ }^{1}$ East China Normal University, People's Republic of \\ China \\ ${ }^{2}$ Oxford University, United Kingdom \\ ${ }^{3}$ University of California, Irvine, United States of America
}

Status. Nonlinear spectroscopy typically uses classical light to probe the material's response functions by varying either frequencies or time delays of the light sources [91]. The obtained signals can be further manipulated by shaping the exciting laser fields, i.e. their bandwidths, polarizations, intensities and laser chirps. We will refer to this scenario as 'classical spectroscopy'. Recent experimental and theoretical research efforts seek to expand this degree of control by exploiting quantum fluctuations of the light fields or by evaluating their information content [28].

Quantum light offers several advantages to spectroscopy -by enhancing signal strengths, by creating new 'control knobs' for the manipulation of optical signals, or by even allowing entirely new types of signals. The strong fluctuations of quantum light can enhance the nonlinear signal strength relative to linear absorption [92]. In addition, time-frequency entanglement of photons can be employed to control excitation pathways and excited state populations in aggregates [93]. Third, the quantum nature of light may be used to study collective effects in many-body systems by back and forth projection of entanglement from the field onto the matter. This allows to prepare and control higher excited states in molecular aggregates, and access dark multiparticle states. Finally, photon coincidence counting experiments can access useful material information imprinted on the quantum statistics of emitted light fields.

The unusual intensity scaling of quantum light signals has been already realized almost thirty years ago, and has been demonstrated to date in atomic and molecular samples. Classical heterodyne $\chi^{(2)}$ signals such as two-photon absorption scale quadratically with the pump intensity and therefore require a high intensity to be visible against lower-order linear-scaling processes. Two-photon absorption of entangled photons, in contrast, scales linearly [94]; nonlinear spectroscopy can be thus performed at much lower photon fluxes (see figure 13). This allows stronger signal detection at lower intensities which has practical applications to imaging of fragile biological samples.

Apart from affecting the signal scaling with intensity, quantum properties of light, and in particular time-frequency entanglement constitute an entirely new paradigm for shaping and controlling exciton dynamics pathways in molecular aggregates in a way that cannot be achieved with shaped classical pulses. This is because between the two absorption events in entangled two-photon absorption, the light and material system are entangled [93]. The entanglement time, which originates from group velocity dispersion in the birefringent crystal provides temporal correlations, whereas the pump bandwidth ensures frequency anticorrelation

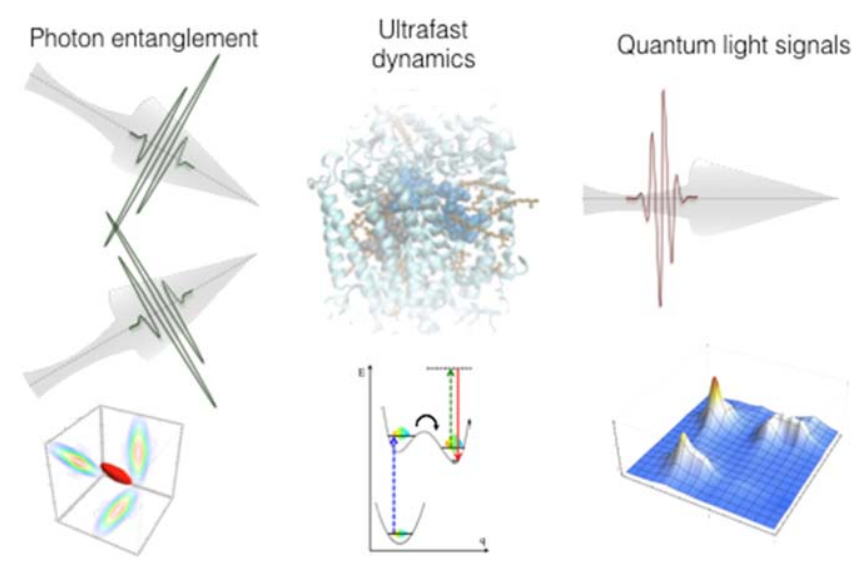

Figure 13. An entangled photon pair probes the ultrafast dynamics in a molecular system via the pump-probe technique. Due to the unique temporal and spectral parameters of entangled light, the spectroscopic signal obtained by quantum light carries molecular information not accessible by classical light.

between entangled photons. By varying the entanglement time and the pump bandwidth it becomes possible to probe subsets excitation pathways via the selection of specific biexciton states in the detected optical signal. Ultrafast relaxation processes in the single exciton manifold limit the classically achievable degree of pathway selectivity due to the trade-off between spectral and temporal resolution for each absorption process. With entangled photons, this trade-off applies only to the overall two-photon process, but not each individual transition. This allows to collect information about matter with high simultaneous temporal and spectral resolution, not possible with classical light [93]. Classical light is fundamentally limited by the frequency-time uncertainty, whereas entangled photons have independent temporal and spectral characteristics not subjected to this uncertainty.

Furthermore, quantum fluctuations of light typically become important when photon numbers are low. Photon coincidence counting experiments can reveal important information about the light emitters. This can be turned into a spectroscopic tool by placing a sample into the beam line of one of the two entangled photons and recording the change in the coincidence count rate. This strategy has been termed biphoton spectroscopy [95]. Such measurements contain linear absorption information, but detected with improved signal-to-noise ratio, as the coincidence counting suppresses dark counts, and hence the noise. Combining biphoton spectroscopy with additional excitation pulses turns this technique into a novel multidimensional nonlinear spectroscopy. The extension of biphoton spectroscopy to the nonlinear regime is challenging due to required alignment between classical pump fields with the quantum single-photon probe fields, which are generated spontaneously.

Current and future challenges. The implementation of quantum light sources as spectroscopic tools faces several challenges. First and foremost, low intensity fields require long signal collection times compared to classical measurement techniques which use much larger photon 
fluxes. This has always been a limiting factor in quantum optics, and is the main bottleneck in the development of, e.g. N00N-states (for large photon numbers $N$ ) for quantum metrology [62], and is indeed a challenge in quantum spectroscopy as well. The implementation of quantum spectroscopy in biological systems requires the combination of expertize in biology and quantum optics.

On a theoretical level, research has mostly focused on the question of how quantum light sources, which were developed predominantly for quantum information applications, can be employed for spectroscopy. Very little attention has been paid to the question of how an 'ideal' quantum light source would look like. In quantum metrology, there are clear answers as to how much a N00N-state can improve the phase resolution beyond the classical limit. To date, despite many new insights into how entanglement affects two-photon absorption, there is no comprehensive understanding as to under which conditions quantum light can enhance spectroscopy, or what kind of quantum states will be best suited for a given spectroscopic task.

It has been pointed out in [96], that any nonlinear optical measurement can be equally considered as a photon counting measurement, where the molecule plays the role of a photon coincidence detector with a more complex internal structure. As such, future experiments might also provide a new avenue for exploiting ultrafast molecular dynamics to characterize multimode states of quantum light [94].

Concluding remarks. Nonlinear quantum-optical signals are described by the convolutions of multi-time correlation functions of field and matter [28]. This viewpoint naturally connects to the established framework of quantum optics, where field correlation functions are analyzed with nonlinear laser spectroscopy, which investigates the information content of matter correlation functions. It provides a flexible platform to explore quantum light interaction with complex systems that goes beyond spectroscopic applications. This could include coherent control with quantum light, or the manipulation of ultracold atoms with light.

Entangled quantum states with larger photon numbers [97] promise access to the $\chi^{(5)}$ susceptibility and its additional information content. The combination of quantum light with strong coupling to intense fields in optical cavities may result in new coherent control techniques of chemical reactions. Quantum light generation and detection should be combined with established methods to extract phase-dependent multidimensional signals [98]. Entangled photons constitute a new, highly versatile tool for nonlinear spectroscopy.

\section{Acknowledgments}

KED is supported by the National Science Foundation of China (Grant No. 11934011) and the Zijiang Endowed Young Scholar Fund and Overseas Expertize Introduction Project for Discipline Innovation (111 Project, B12024). FS acknowledges funding from the European Research Council under the European Union's Seventh Framework Programme (FP7/ 2007-2013) Grant Agreement No. 319286 Q-MAC. SM gratefully acknowledges the National Science Foundation (grant CHE-1663822) and the U.S. Department of Energy, Office of Science, Office of Basic Energy Sciences under Award DE-SC0020168, which supported KED collaborative visits to UCI. 


\section{Interacting a handful of quantum emitters and photons}

\section{Vahid Sandoghdar}

\section{Max Planck Institute for the Science of Light, Germany}

Status. Early spectroscopy in the nineteenth and twentieth centuries was based on the interaction of an ensemble of material and light particles. Starting with flames, bulk matter or atomic and molecular cells on the one hand and sun light, incandescent or discharge lamps on the other, scientists continuously improved their experimental toolbox to acquire more control over the inherently statistical nature of these systems. Inventions of molecular and atomic beams as well as lasers were decisive steps in the middle of twentieth century towards well defined systems followed by the development of ion traps and atom cooling and trapping. These efforts were finally taken to the limit, where a range of material could be detected at the single emitter level, including atomic ions and neutral species in vacuum, organic molecules and semiconductor quantum dots on surfaces or in bulk, color centers in diamond, defects in carbon-based nanostructures, and rare earth ions in crystals.

Parallel to the endeavors on taming single material particles, techniques were developed for generating single photons, e.g. from single atoms and molecules. In addition, various approaches were pursued toward the production of squeezed light and entangled photons. Routine interaction of such forms of nonclassical light and single quantum emitters, however, remains scarce mainly due to low efficiencies and the heavy experimental preparation steps required. Nevertheless, isolated experiments have demonstrated the coupling of single photons and emitters both in free space and in microcavities.

The mastery of matter and light at the fundamental quantum level marks an impressive milestone in the progress of quantum optics, but controlled up-scaling of the number of quanta will be the next Holy Grail for making quantum optical phenomena accessible to science and technology. Large networks of well-defined quantum emitters efficiently coupled to each other via photons can be used for quantum information processing, but more importantly, they give access to complex quantum systems where nontrivial manybody and cooperative effects play a central role. These are important building stones of the emerging field of quantum engineering.

Current and future challenges. A crucial many-body coupling of photons and quantum emitters is efficiency. In other words, for a complex light-matter quantum state to become practical, each photon has to be 'wired' to each emitter efficiently. As this intuitive picture suggests, the key lies in proper engineering of the photonic modes. Perfect coupling to a dipolar transition requires a dipolar field [99], which is not a trivial optical mode to prepare. To get around this challenge, one can take advantage of the fact that the radiation pattern of an emitter can be modified next to an interface. For example, a subwavelength waveguide (nanoguide) can act as an optical antenna to match the radiation pattern of an emitter in its evanescent vicinity to its own spatial mode, thus, allowing for efficient coupling of a nanoguide photon to the emitter [100-103]. This promises a scalable architecture for a quantum photonic network, albeit with several challenges of its own.

First, fabrication of solid-state emitters such as quantum dots, color centers, ions or molecules within small tolerance margins, especially at well defined locations and in combination with photonic micro- and nanostructures, continues to be extremely difficult. Indeed, while a variety of microcavities, photonic crystals, nano-antennas and similar structures has been used in the past three decades to tailor the electromagnetic field and enhance the photon-emitter radiative coupling, their routine implementations confront many technical and material issues, be it semiconductor, diamond, or organic [104, 105].

A more formidable future challenge on the material side will be the control of phononic interactions in the condensed phase. Coupling to the large number of degrees of freedom of individual atoms and molecules in a solid, leads to nonradiative decay as well as dephasing, reducing the optical cross section of an emitter. In addition, spectral jumps can cause instability over time. This is why solid-state quantum optical experiments are bound to low cryogenic temperatures. Thus, it would be extremely fruitful if one could engineer the phononic bath in a solid in a similar fashion as is done by microcavities or nano-antennas in the optical domain to inhibit some of the phononic couplings. Another intriguing avenue would be to speed up the radiative decay to a sufficient degree that it becomes comparable or faster than dephasing processes, which are typically in the femto- to picosecond range.

Among the possible quantum materials to explore these ideas, molecules have some decisive advantages [100, 101, 105]. First, they enjoy the highest fabrication quality. Second, they are very small so that local field enhancements, e.g. in nano-antennas, can be optimally exploited. On the downside, molecules remain sensitive to interactions with their environment because of the large number of internal degrees of freedom associated with vibrations. However, this curse could also be turned around to a virtue if one succeeds in mastering phononic interactions because then one could exploit the rich energetic landscape for various quantum operations.

Generation of nonclassical light is also highly nontrivial. Ideally, one would like to dial in key parameters such as frequency, bandwidth and brightness to suit specific applications. In addition, triggered operation is often desirable for deterministic synchronization of various processes. However, sources that deliver Fock states, entangled or indistinguishable photons, and squeezed light are usually very limited in performance. More exotic quantum states of light are even farther from reach.

A great deal of pioneering works on nonclassical light has been done using nonlinear processes such as parametric down conversion. More recently, promising progress has 
been made in the development of single photon sources from single quantum emitters such as organic molecules and semiconductor quantum dots. By coupling the emitter to a resonant or non-resonant photonic structure such as an antenna, emission rates up to several ten million photons per second have been demonstrated. However, choices of operation frequency and bandwidth are still very limited. The experimental bottlenecks are often the same as those of the emitter technology such as high spectral quality of an emitter coupled to a nanophotonic structure. As a result, spectroscopy with nonclassical sources of light has been demonstrated only in few proof-of-principle studies [54, 106]. Establishment of multi-photon and multi-emitter networks poses a great challenge today.

\section{Advances in science and technology to meet challenges.}

The past three decades witnessed many exciting experimental developments that have allowed a laboratory mastery of light and matter at the single particle level. Understandably, these efforts usually aimed at first demonstrations and were not meant to deliver robust technological platforms. It is foreseeable that many of these developments, e.g. singlephoton sources, make their way to the market, thus making high-end experiments available to a larger scientific community, much like the laser industry has continuously done since the 1970s. However, a huge amount of fundamental research still lies ahead before quantum engineering can get a handle on decoherence sources and protect the 'quantumness' of the system.

Drawing an analogy to the micro-electronics industry, we need a similar degree of purity and fabrication functionalities as has become available in silicon after a tremendous investment of resources. Today, while many material platforms such as diamond are being explored in a fury, the 'silicon of quantum photonics' remains yet to be identified. Thus, investment in chemical synthesis of new molecules as well as growth and doping of various organic and inorganic crystals will be very valuable. Combined with powerful structure and function calculations, these could provide us with optimized designer materials, where phononic couplings and decoherence sources are controllable. In addition, the road to complex quantum networks will most probably involve hybrid architectures, where electronic, vibrational and spin degrees of freedom are coupled to each other. This imposes further demand on the combination of different materials on one platform.

\section{Concluding remarks}

A decisive breakthrough for the future of quantum technologies will be the development of robust strategy for interacting several quantum systems via photonic channels. To achieve this, one requires well-behaved quantum emitters, practical sources of nonclassical light and efficient wiring of photons and quantum emitters. This means going well beyond proof-of-principle experiments, where simple concepts that are well understood in quantum mechanics are demonstrated in the laboratory. The field rather needs long-term and largescale efforts to tackle material challenges discussed above. To make this possible, a new discipline of quantum engineering has to merge with strong elements from solid-state physics, quantum optics, nanoscience and nanotechnology as well as material science. Much along the lines of what Feynman stated in his insightful presentation in 1959 [107], the dream remains to control the individual particles in matter. In addition, however, the quantum optics community will need to develop analogous efforts to control and sculpt light at the single photon level.

\section{Acknowledgments}

I am grateful to many talented group members who have contributed to our work over the past twenty years. I also acknowledge the generous support of the Max Planck Society. 


\section{Nonlinear optical coherent spectroscopy with EPPs}

\section{Michael G Raymer and Andrew H Marcus}

University of Oregon, United States of America

Status. In recent years, a number of seemingly unrelated research themes have converged to define the idea of using quantum EPPs to perform two-dimensional optical coherent spectroscopy [28, 108]. In the field of nonlinear optics, methods to study the coherent dynamics of atoms and molecules using 'classical' femtosecond laser pulses are well established. Recently, new techniques have emerged to enable such measurements under conditions of low laser fluence and small signals. For example, the technique of two-dimensional fluorescence spectroscopy (2DFS) [109] was developed, in which the system is excited by a train of four femtosecond laser pulses and the ensuing photo-induced fluorescence is detected as a function of the inter-pulse time delays and phases. 2DFS is a type of two-dimensional electronic spectroscopy (2DES) [110], which measures the third-order nonlinear optical response of a material system $\chi^{(3)}\left(\omega_{p}, \omega_{q}\right.$, $\left.\omega_{r}\right)$, where the independent variables are optical frequencies. While nearly all methods to determine $\chi^{(3)}$ rely on impulsive excitation of the system using ultrashort laser pulses, it is known that broadband incoherent (thermal-like) light can approximate ultrashort pulses due to its short coherence time. This principle was applied to carry out certain nonlinear spectroscopic experiments using incoherent light as the excitation source, such as optical photon-echoes and 2DES [111].

In the field of quantum optics, the temporal and spectral correlations of EPPs generated by SPDC were explored for a monochromatic continuous-wave pump laser [112], and a broadband ultrashort pump laser [113]. In both cases, the individual photons that comprise a photon pair can have a very broad spectrum (e.g. $\sim 40 \mathrm{~nm}$ in wavelength) and a corresponding ultrashort coherence time (e.g. $\sim 15 \mathrm{fs}$ ). Although the intrinsic temporal and spectral correlations of the EPP state distinguish it from broadband thermal-like light (where no inter-photon correlations are present), it was recognized that this property of EPP could be exploited potentially to probe the nonlinear optical response of material systems in ways that are not possible using classical light sources. The developments discussed above inspired a series of papers by the Mukamel group, which proposed to use EPP to replace the femtosecond laser pulses in conventional nonlinear spectroscopy [28].

In light of Mukamel's proposed EPP schemes, it is useful to consider the concepts demonstrated by two-photon interference experiments, a novel form of which were pioneered by Franson [114]. In Franson's studies, the individual photons of the EPP were directed into separate, unbalanced Mach-Zehnder interferometers (MZIs), and their transmission at the exit ports were monitored using singlephoton detectors. It was found that the photon coincidence count rate depended on the phase of either MZI, while the rate

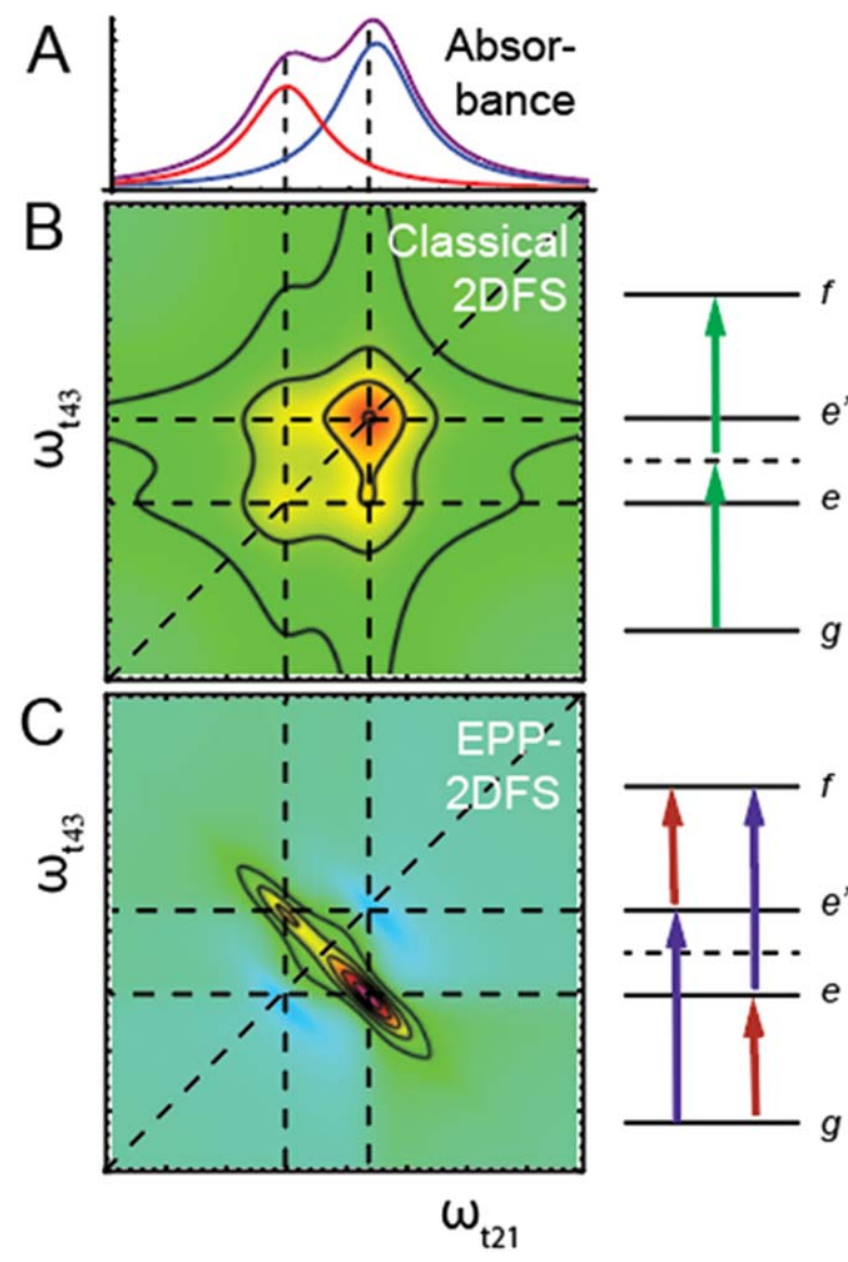

Figure 14. Simulated absorbance (A), classical 2DFS (B), and EPP2DFS (C) for an electronically coupled molecular dimer. For both 2DFS and EPP-2DFS, transition energy level diagrams are shown on the right. The temporal-spectral correlation of the EPP state effectively 'windows' the classical 2DFS spectrum, facilitating the detection of cross-peak features. Adapted from [108]. Reprinted figure with permission from [108]. Copyright 2013 American Chemical Society.

of individual photons was independent of these phases. Such behavior is a reflection of quantum entanglement, which is the key resource in quantum technologies.

The concepts described above form the basis of a proposed scheme to use a Franson two-photon interferometer and EPPs to enhance the performance of 2DFS [108]. In the proposed 'EPP-2DFS' experiments, the photons exiting the MZIs are used to excite a two-photon-resonant material system, and the ensuing fluorescence is detected. The enhancements predicted theoretically with the proposed method are: (i) the ability to probe samples at very low light levels (an advantage for samples prone to photo-damage); (ii) a higher signal-to-noise ratio for a given incident light flux; and (iii) increased spectral resolution and ability to detect 2D spectral cross-peaks resulting from the temporal-spectral correlations of the EPP state (see figure 14).

Current and future challenges. In order to build a functional EPP-2DFS instrument, it is necessary to address significant practical challenges. Of particular importance is the creation and 
control of the EPP state incident at the sample. The individual photons of the EPP must travel along separate, controllable pathways (in terms of time delay and phase) before arriving simultaneously at the sample with minimal wave-packet dispersion. Because EPP fields are much weaker than those used for classical light experiments, techniques for balancing interferometers and testing dispersion-compensation optics must be implemented using high-temporal-resolution photoncounting detectors configured in a cross-correlation scheme. Another challenge is the need to detect the fluorescent photons emitted from the sample as a function of the phases of the wave-packet components of the EPP field. It is therefore necessary to implement phase-sensitive photoncounting detection in the low-fluence regime, which can be accomplished using fast time-tagging electronics in combination with field-programmable gated arrays.

On the fundamental side, it will be necessary to prove theoretically, and to verify experimentally, that quantumenhanced spectroscopy outperforms classical light spectroscopy in important situations. For example, it is possible to prepare classically correlated light fields that simulate to some extent the behavior of EPP in the Franson interferometer [115]. However, the interference visibility of the coincidence count rate is limited for classical light fields, whereas $100 \%$ visibility is predicted theoretically in the quantum case [114]. Similarly, while theory predicts that the time-frequency entanglement provides enhanced spectral resolution, it remains to be tested whether or not suitably designed classical fields can simulate this effect.

Advances in science and technology to meet challenges. In EPP-2DFS, the molecular sample acts as a simultaneous timefrequency coincidence detector, responding in a manner not subject to the standard (single-photon) time-frequency uncertainty principle [108]. As an example, a coupled molecular dimer exhibits a nonlinear two-photon absorption process that is otherwise absent in the uncoupled monomers [116]. The resulting enhancements of the EPP-2DFS measurement in such systems are expected to be in the precision of extracting molecular Hamiltonian parameters and the temporal dynamics following optical excitation. In order to ensure that the results of EPP measurements are as meaningful as possible, it will be necessary to test and verify the measurement scheme by developing tailored molecular systems with well-characterized model Hamiltonians whose coupling parameters can be systematically adjusted. Excitoncoupled molecular dimers in DNA scaffolds are one promising system for this purpose [117]. Nevertheless, further research is needed to develop additional systems with suitable optical properties.

Concluding remarks. A possible application for quantumenhanced nonlinear spectroscopy is to facilitate control of quantum states in material systems. This might be accomplished using tailored EPP states, which can be generated using coherently shaped ultrashort laser pulses as the pump field for generating the EPP. Such states are predicted to enhance two-photon absorption probabilities [118], and might be used to create entangled states of monomers within a coupled molecular dimer, trimer, or larger complex. To fully exploit these and other 'quantum advantages' brought by EPP, it will be necessary to develop efficient protocols to fully characterize EPP states. Although quantum state tomography of femtosecond-pulse-generated EPP has recently been demonstrated $[119,120]$, these methods are yet to be applied to quantum light spectroscopy.

\section{Acknowledgments}

We thank Brian Smith for useful discussions. This work was supported by the John Templeton Foundation (RQ-35859 to MGR and AHM), by the National Science Foundation RAISE-TAQS program (PHY-1839216 to MGR and AHM), and by the National Science Foundation Chemistry of Life Processes program (CHE-1608915 to AHM). 


\section{Entangled photon nonlinear spectroscopy in organic and biological molecules}

\section{Oleg Varnavski, Theodore Goodson II' \\ University of Michigan, United States of America}

Status. As the need for improved detection methodologies for photonic, medical and remote sensing applications continues to intensify there has been increased attention given to unconventional approaches. In particular, the use of quantum sensing techniques where the sensitivity may exist below the standard quantum limit have been recently enthusiastically investigated. Techniques which utilize nonclassical (entangled) light for enhanced sensitivity as well as exploration of new mechanisms of light-matter interaction have obtained great interest for their possible discoveries in physics, chemistry, biology, and in medicine. In this roadmap, the use of nonclassical states of light for enhanced nonlinear optical effects in organic and biological systems is discussed. The results of the initial studies in these areas will be summarized from entangled nonlinear optical investigations. This roadmap also takes a look at the very promising new directions in this area which could lead the way to new spectroscopic tools as well as microscopic approaches with new information about the nature of nonclassical light interactions with matter.

While the use of entangled light for the interaction of photons in chemistry and materials is still relatively new, there have been previous reports of squeezed states of light interaction with matter in the past. Work with squeezed light and other highly correlated sub-poissonian statistics beams of light have illustrated the unique features of enhancements of nonlinear optical effects. With the process of SPDC the generation of photons entangled in polarization and frequency was illustrated as a very powerful tool to practically create this form of non-classical light in the laboratory. Out of these interesting studies grew interest in creating theoretical models of the interaction of entangled photons with electronic states of matter. The possibility of virtual state spectroscopy was presented as a new way to probe and potentially control the excitations in atomic and molecular systems. The first organic molecular system investigated for the possibility of entangled two photon absorption was done in a porphyrin dendrimer system [121]. The very large enhancement in the crosssection and the linear dependence on the EPPs in a nonmonotonic quantum interference manner led to the direction of doing sensitive nonlinear optical spectroscopy with a very small number of photons [122-124]. Further studies illustrated the sensitivity of the entangled photon nonlinear optical process to the phase matching conditions of the experimental arrangement [125], as well as the unique property of the entanglement delay providing information regarding the spectral response of the virtual states accessible in the organic material $[94,121,122]$. These studies, along with applications in biological protein systems [126] as well as in the electronic structure of donor-acceptor chromophores

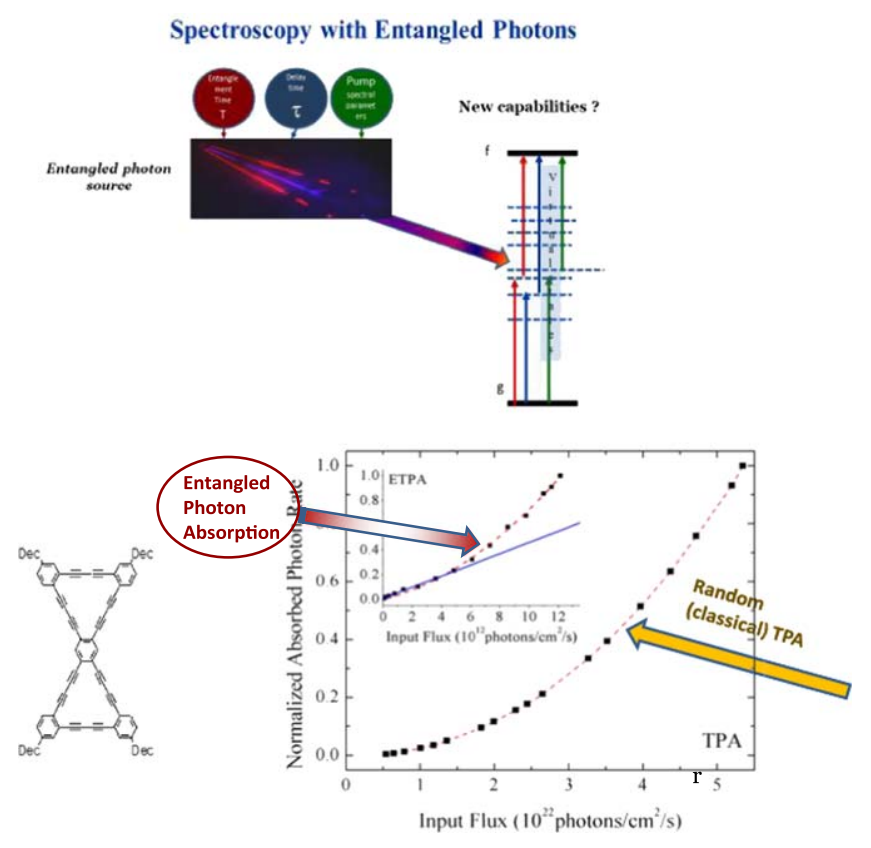

Figure 15. Entangled photon spectroscopy and the difference in the entangled and classical two photon effects in an organic chromophore.

[127] provided just the beginning of what is hoped to be a new and powerful approach to spectroscopy.

Current and future challenges. With the initial publications and enthusiasm for utilizing entangled states of light to probe nonlinear optical transitions in molecules new ideas and challenges have been presented which may push the capabilities of entangled photon nonlinear spectroscopy into the next generation. Heavily conceived by the theoretical models predicted by Mukamel et al [127] the possibility of furthering the use of entangled photon excitation beyond the case of two-photon absorption have motivated scientists to think deeper regarding the realization of new spectroscopic tools involving quantum light. One of the major thrusts of research in this area involves not only the ability to detect nonclassical states of light with molecules but also to possibly control the excitation or population of excitation states as well. With the unique property of entanglement between two photons it may be possible to populate various states selectively as well as enhance the population of particular states [128]. Indeed, this possibility could have enormous applications in chemistry, solar photochemistry (photosynthesis), as well as in the detection of various materials in the presence of a noisy background. There have been reports which have pushed this approach forward. For example, reports of EPP-2DFS, to sensitively probe the nonlinear electronic response of molecular systems have utilized a separated two-photon ('Franson') interferometer to generate time-frequency-EPPs, into the framework of a fluorescence-detected 2D optical spectroscopic experiment [129]. In comparison to 'classical' 2DFS techniques, major advantages of this scheme are the suppression of uncorrelated background signals, the enhancement of simultaneous time- 
and-frequency resolution, the suppression of diagonal 2D spectral features, and the enhancement and narrowing of offdiagonal spectral cross-peaks that contain information about electronic couplings [129]. These effects are a consequence of the pure-state field properties unique to a parametric downconversion light source, which must be included in the quantum mechanical description of the composite field-molecule system. For complex systems the convolution of field and matter responses strongly affects the excitation pathways as well as time and frequency resolution that can be obtained. Entangled photons have independent temporal and spectral characteristics which are not Fourier conjugate which allows for a timefrequency resolution not achievable with classical light [128]. This non-classical bandwidth property of entangled light opens new ways of controlling the population of short-lived states and selection of unique ptotochemical pathways. A combination of quantum entangled light with interferometric techniques has been suggested to substantially increase the resolution and selectivity of Raman signals [128]. This approach can better reveal detailed molecular information and is very promising for future sensing applications. While the field of entangled photon nonlinear spectroscopy is still at the beginning of this journey, the future centered around the possibility of learning new information with new control knobs regarding the interaction of entangled light with matter is very bright. For example, there is a relatively new push to provide the opportunity to investigate not only organic molecules with new selectivity of entangled photons for spectroscopy but to also provide a microscopic analysis with this methodology. In doing so this would drastically reduce the number of photons necessary for excitation which is a major goal for microscopic nonlinear optical investigations of biological systems. The reduction in the photon flux necessary for excitation with entangled photons in comparison to the classical case can be several orders of magnitude which offers new opportunities for characterization of delicate biological systems.

Advances in science and technology. Moving forward there are important challenges which must be met in order to realize the goals of revolutionizing the field of molecular spectroscopy with the use of entangled photon excitation. A major challenge is to develop a method to characterize and predict the electronic interactions of the EPPs in molecular systems. While the general methodology of electronic structure can provide general information, more specific and detailed information involving the participation of virtual states is not trivial. In many ways predicting the nonmonotonic quantum interference interactions of electronic states may be the key toward discovering the hidden attributes of entangled light approaches to spectroscopy, imaging and sensing.

Another challenge the field of spectroscopy faces based on non-classical states of light is that current experimental development currently lags behind the rapidly expanding theoretical efforts. In order to push experimental and device development forward, new high flux and high entanglement quality sources of entangled photons should be developed.
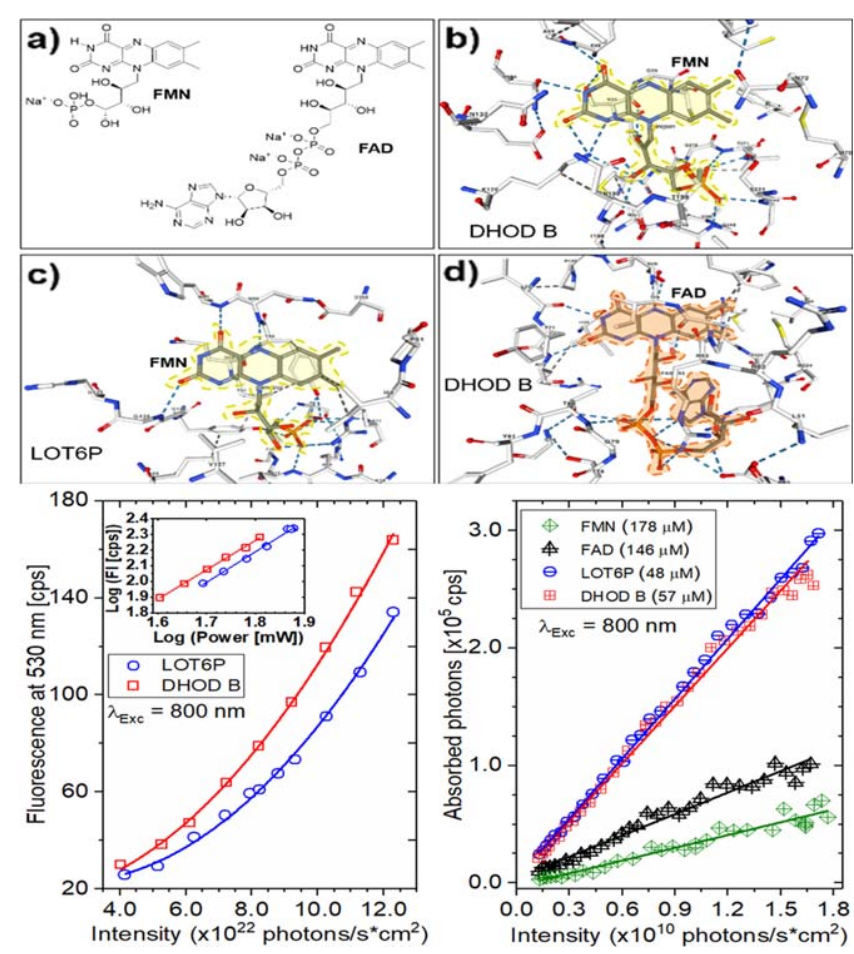

Figure 16. Entangled two photon effects in a biological protein system. Reprinted with permission from [126], Copyright 2018 American Chemical Society.

Nonlinear optics with extremely low light intensities offers an enormous promise in spectroscopic and sensing capabilities, but at the same time, it requires substantial improvement of the detection methods and data handling procedures dealing with a small number of photons. Single photon detectors with high quantum efficiency and low dark count noise need to be developed. A coincidence detection approach is one of the most important specific methods being used in non-classical light spectroscopy and sensing. It requires substantial improvement in terms of the time width of coincidence time window. It may include the ultrafast classical light optical gating to provide femtosecond time resolution [120]. In order to perform spectroscopic and microscopic experiments with low number of detected photons in a reasonable time, multichannel systems with parallel processing of the data are preferable. The development of the detection systems optimized for the specific detection and measurements procedures utilizing entangled photons is in its infancy.

Concluding remarks. This new field of using quantum light in nonlinear spectroscopy constitutes an entirely new approach to shaping and controlling excitation pathways in molecular systems that cannot be achieved using classical light. This rapidly developing scientific and technological direction offers novel control parameters and unprecedented selectivity and sensitivity in obtaining novel spectroscopic information. The theoretical models, experimental results of entangled nonlinear signals, and development of the enabling 
technologies necessary to push the field forward are impressive. The potential applications for the detection of new quantum light-matter interactions are very promising and could provide new insights into a number of scientific and technological fields.

\section{Acknowledgments}

We thank the National Science Foundation (CHE1607949) and Air Force Office of Scientific Research (FA9550-1710457) for support of this research. 


\section{Characterizing optical properties of chiral materials with twisted photonic states}

\section{Zhi-Yuan Zhou and Bao-Sen Shi}

University of Science and Technology of China, People's Republic of China

Status. Electromagnetic waves have both spin angular momentum (SAM) and OAM [130]. SAM produces polarization. The OAM arises from the helical phase structure in the paraxial regime. Photon with OAM can be regarded as helices with left- and right-handedness twisted to varying degrees. Light carrying OAM has broad applications in microparticle manipulation, high-precision optical metrology, and potential high-capacity optical communications [131]. Besides, light carrying OAM has been used to measure the chirality of molecules or materials with chiral symmetry [132], which may find interesting applications in sensing and chiral spectroscopy. Recently, it was found that using quantum light to measure the rotatory dispersion of chiral molecules could obtain more information gain and higher sensitivity than that using classical lasers [133], showing a promising way for quantum-enhanced measurements of the chirality with potential applications in chemistry, biology, materials science, and the pharmaceutical industry. Additionally, optical metrology with quantum light also opens up new opportunity in spectroscopy, as quantum light shows non-classical correlations in different degree of freedoms such as frequency, polarization, linear momentum, angular momentum and photon numbers, these correlations would lead to different scaling factors and higher sensitivity beyond standard quantum limits [28]. So using quantum twisted photons presents itself naturally and may pave a new way to measure the chirality of materials with surpassing standard quantum limits.

In order to measure the chirality of materials with quantum twisted photons, we have to generate these photons on request firstly. So far, SPDC in a second-order nonlinear crystal [134] and spontaneously four-wave mixing (SFWM) in a third-order nonlinear medium such as fiber or atomic ensemble [135] are two very common and mature ways for effectively generating quantum EPPs. Due to the conservation of OAM in both ways, the generated two-daughter photons are entangled in OAM degree of freedom (figure 17). Besides, people can transform spin entangled photon sources to OAM entangled sources in a two-dimensional subspace by using spin-orbit coupling. There have been many progresses achieved on this topic for many years [136]. After we have an OAM entangled photonic source, we can use it to characterize chiral materials.

Current and future challenges. Previous investigations show that although light carrying OAM cannot interact with the chiral material through electrical or magnetic dipoles, it does not exclude the possibility of interaction through higher order interactions such as electric quadrupole fields (EQFs) [133]. Of course, the interaction strength of EQFs is much weaker than electrical or magnetic dipoles. Various studies on
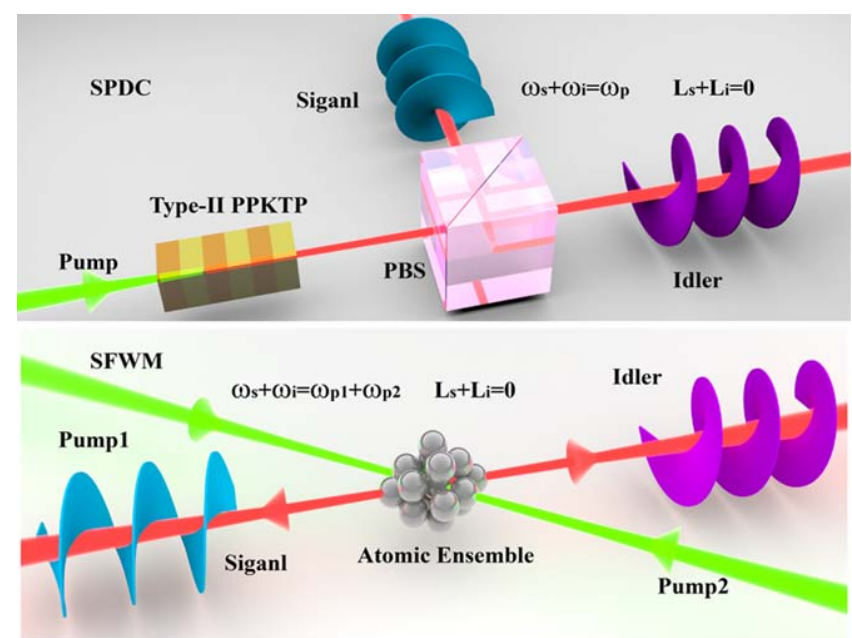

Figure 17. Generating OAM entangled photons in SPDC and SFWM with Gaussian pump beams. Due to the conservations of energy and OAM, a pump photon with frequency $\omega_{\mathrm{p}}$ is split probabilistically to signal and idler photons with a frequency of $\omega_{\mathrm{s}}$ and $\omega_{\mathrm{i}}\left(\omega_{\mathrm{s}}+\omega_{\mathrm{i}}=\right.$ $\left.\omega_{\mathrm{p}}\right)$, and topological charges of $L_{\mathrm{s}}$ and $L_{\mathrm{i}}\left(L_{\mathrm{s}}+L_{\mathrm{i}}=0\right)$ in SPDC. For SFWM, there are two pump beams, and the conservations of energy and OAM result in relations of $\omega_{\mathrm{s}}+\omega_{\mathrm{i}}=\omega_{\mathrm{p} 1}+\omega_{\mathrm{p} 2}$ and $L_{\mathrm{s}}+L_{\mathrm{i}}=0$.

investigating the interaction between the chiral materials and light carrying SAM or quantum light source entangled in SAM have been performed, but just a few works focus on the study with light carrying OAM, and there have been no reports on investigating the interaction between the chirality of materials and quantum light entangled in OAM. At present, the underlying physics of the light-matter interaction between the chiral materials and light with OAM is still not clear. How the chiral material responses to light carrying different OAM topological charges still needs various explorations. Besides, the works using quantum light to explore the chiral property of the materials are very rare, more investigations should be performed in order to reveal the distinguished features of quantum optical metrology, such as different scaling factors, higher resolution and sensitivity by using a two-photon or a single-photon detection. Besides, for measuring the chirality of the materials with quantum photons entangled in OAM, the bandwidth, wavelength and topological charges of the quantum entangled photons should match a specific material. So there are two main challenges needed to be addressed: 1 . establishing a mathematical model to quantitatively describe the interaction between OAM entangled photons and various chiral materials, and to figure out the underlying physics; 2. experimentally generating OAM entangled photons with proper parameters of central wavelength, bandwidth, topological charges to match a specific material under study. That means we should have the ability to change the central wavelength, topological charges and the spiral bandwidth of the quantum-entangled photons at will during the preparation. In addition, some assisting technique such as an interferometric method may be used to enhance the sensitivity in the measurement, so how to establish an effective quantum optical model to describe the measurement parameters in different interference configurations should be considered. 
We will show below that these challenges can be overcome in the near future.

\section{Advances in science and technology to meet challenges.}

Usually the semi-classical or the full quantum theory is used to study various light-matter interactions; it can definitely be used to describe the interaction between light with OAM or quantum photons entangled in OAM space and the chiral materials. What we should pay more attention to are the special dynamics with the transverse optical field distributions and the special symmetry provided by the specific chiral material when we use these theories. The growing interests in optical metrology with quantum light will stimulate new experimental studies on this topic, and the underlying physics will become much clearer in the near future. For generating OAM entangled photons on request, we can change the central wavelength, bandwidth, brightness and topological charges of the source at will by controlling parameters of the nonlinear media and of the pump lasers. For example, we can adjust the spiral bandwidth of quantum photons generated via SPDC by changing the length of the nonlinear crystal or the beam size of the pump laser, therefore it is feasible to generate OAM entangled photons matching a specific material. It is quite easy to transform a spin entangled photonic state to an OAM entangled photonic state in a two-dimensional subspace through spin-orbit coupling by using a commercial spatial light modulator or a nano-fabricated spiral phase mirror, respectively. So far, the maximum topological charges of 300 and of more than 10000 have been achieved experimentally. It is also possible to generate the entangled photons at special wavelength, for example the wavelength in the mid-infrared or ultraviolet regime, which is very hard to generate using a traditional way due to the lack of a suitable pump laser or an available nonlinear medium. The technique of quantum frequency conversion can be used to change the wavelength of photons easily [137]. We can use quantum photons assisted with an interferometer such as a Mach-Zendner interferometer to enhance the resolution and sensitivities in the measurement. A scheme for measuring the thermal dispersion coefficients of the two principal refractive axes of a birefringent crystal with a single measurement has been experimentally verified [138]. This method can be used to measure the optical properties of the chiral material by using quantum twisted photons and by replacing the birefringent crystal inside with a specific chiral material (figure 18). At present, the most OAM entangled photonic states generated are two-photon entangled states, a multi-photon OAM entangled state may show more distinguished ability such

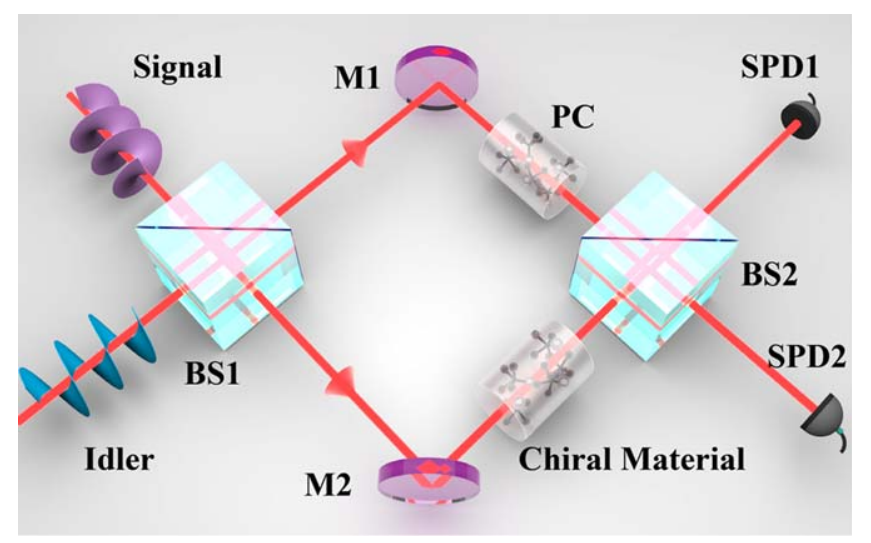

Figure 18. A proposed scheme for probing the chirality of materials by using a Mach-Zehndner interferometer with OAM entangled photons. The photons are firstly split by the beam splitter 1 (BS1), then interact with the chiral material in one arm of the interferometer. In other arm, a dielectric plate (PC) with a proper thickness is used to compensate the optical path difference. Finally, the two beams combine in a second beam splitter (BS2). The outputs from BS2 are detected with two single photon detectors (SPD 1,2) for coincidence measurement. The coincidences against phase change will reveal the optical properties of the chiral material.

as higher resolution and sensitivity in studying the chirality of materials, like done by using a NOON state to measure the phase in quantum optics.

Concluding remarks. People are becoming more and more interested in measuring the chirality of materials with OAM entangled photons, because it may open a promising way for quantum-enhanced measurements of chirality with potential applications in many fields, such as chemistry, biology, materials science, and the pharmaceutical industry so on. With the rapid developments of techniques in quantum metrology and in the manipulation of OAM entangled states, the promising advantages such as different scaling factor, higher resolution and sensitivities of chirality measurement with quantum light illumination may be obtained in the near future.

\section{Acknowledgments}

Z Y Zhou thanks Dr Mukamel for very valuable discussions. This work is supported by The Anhui Initiative in Quantum Information Technologies (Grant No. supports this work AHY020200) and National Natural Science Foundation of China (Grant Nos. 61435011, 61525504, and 61605194). 


\section{Quantum diffraction imaging with entangled photons}

\section{Shahaf Asban ${ }^{1}$, Konstantin E Dorfman ${ }^{2}$ and Shaul Mukamel}

${ }^{1}$ University of California, Irvine, United States of America

${ }^{2}$ East China Normal University, People's Republic of China

Status. Entangled states of light offer a novel tool for diffraction measurements. Consider the setup depicted in figure 19 [139]. An EPP is generated using parametric down conversion and split into two beams. One beam (the signal) is scattered off a sample and then collected into a bucket detector, while the other (idler) is scanned spatially in the transverse plane. Both photons are finally detected in coincidence. There are number of merits in this quantum diffraction scheme. First, while diffraction images obtained using classical sources requires even number of light/matter interactions, quantum sources also contain odd orders and are therefore phase-sensitive $[139,140]$. The phase carries valuable structural information, e.g. circumventing the crystallographic phase problem. Moreover, transition charge densities are complex quantities, their image can only be fully obtained in a direct manner using quantum sources. Last, the detected diffraction pattern of quantum sources scales as $\propto I_{p}^{1 / 2}$, where $I_{p}$ is the intensity of the source. Classical diffraction scales as $\propto I_{p}$. This promotes quantum sources as valuable candidates for weak-field imaging techniques, avoiding damage to delicate samples.

The quantum diffraction signal can be understood using the Schmidt decomposition of the entangled photons amplitude $\Phi\left(r_{s}, r_{i}\right)=\sum_{n} \lambda_{n} u_{n}\left(r_{s}\right) v_{n}\left(r_{i}\right)$, where $\lambda_{n}$ are the weights of the respective spatial modes $u_{n}(r)$ at the transverse positions $r_{s / i}$ of the signal and idler detection plane. The modal distribution is normalized according to $\sum_{n} \lambda_{n}^{2}=1$, hence $\lambda^{2}{ }_{n}$ can be interpreted as a probability for detection of the respective mode. The initial reduced density matrix of the idler- $\rho_{i}=\operatorname{tr}_{\mu s} \rho_{\mu, s, i}$ obtained by tracing over the signal beam —is diagonal in the Schmidt basis,

$$
\rho_{i}(0)=\sum_{n, i, i^{\prime}} \lambda_{n} v_{n}^{*}\left(\boldsymbol{k}_{i}\right) v_{n}\left(\boldsymbol{k}_{i}^{\prime}\right)\left|\mathbf{1}_{i}\right\rangle\left\langle\mathbf{1}_{i^{\prime}}\right| .
$$

After the signal beam interacts with the sample, the reduced density matrix acquires off-diagonal elements and takes the form

$$
\rho_{i}^{(1)}=\sum \mathscr{P}_{n m} v_{n}^{*}\left(\boldsymbol{k}_{i}\right) v_{m}\left(\boldsymbol{k}_{i}^{\prime}\right)\left|\mathbf{1}_{i}\right\rangle\left\langle\mathbf{1}_{i^{\prime}}\right|+\text { h.c. },
$$

where $\mathscr{P}_{n m}=i \beta_{n m} \sqrt{\lambda_{n} \lambda_{m}}, \sigma(r)$ is the charge density of the target object, and $\beta_{n m}=\int d \boldsymbol{r} u_{n}(\boldsymbol{r}) \sigma(\boldsymbol{r}) u_{m}^{*}(\boldsymbol{r})$ assuming a small-angle scattering approximation. Clearly, equation (2) unlike equation (1), has off-diagonal terms which are depicted in figure 20 . The coincidence signal, defined by the idler-signal

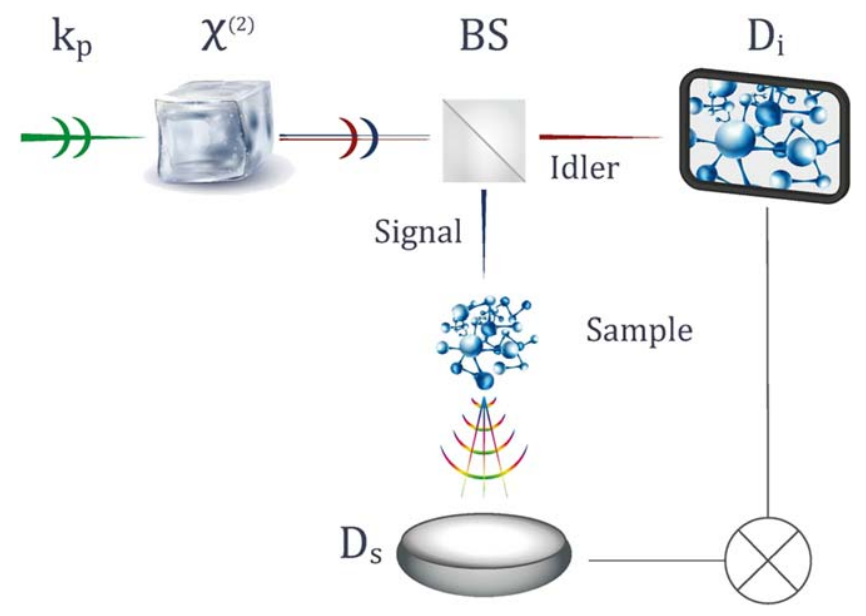

Figure 19. The quantum imaging setup. A broad-band pump $k_{p}$ propagates through a $\chi^{(2)}$ crystal, generating an entangled photon pair denoted as signal and idler. The photons are distinguished either by polarization (type-II), or frequency (type-I) and are separated by a beam-splitter (BS). The signal photon interacts with the sample, then can be further frequency dispersed and collected by a 'bucket' detector $D_{s}$ with no spatial resolution. The idler is spatially resolved in the transverse plane by the detector $D_{i}$. The two photons are detected in coincidence.

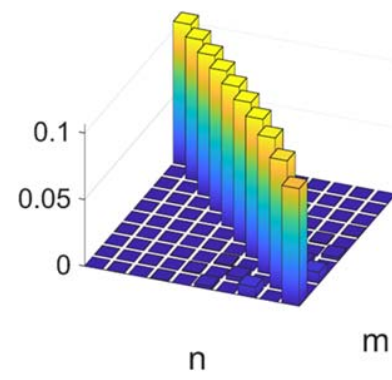

(a)

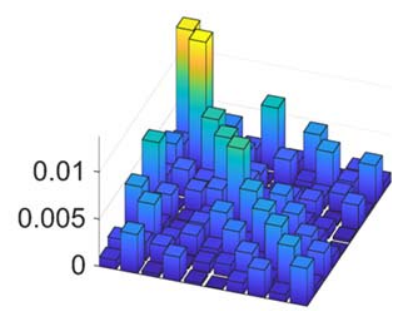

n

(b)
Figure 20. The reduced density-matrix of the idler beam in the Schmidt basis using Hermite-Gaussian modes. (a) The idler's reduced density matrix in equation (1) before the interaction with the object presented in Hermite-Gauss basis modes. (b) The change in the reduced density matrix of the idler due to the interaction with the object in equation (2). The induced coherence (o-diagonal elements) reveal matter information transfer from the signal to the idler.

intensity correlation function takes a similar form,

$$
S\left|\boldsymbol{r}_{i}\right| \propto \mathfrak{R e} \sum_{n m} \sqrt{\lambda_{n} \lambda_{m}} \beta_{n m} v_{n}^{*}\left(\boldsymbol{r}_{i}\right) v_{m}\left(\boldsymbol{r}_{i}\right)
$$

This signal has no classical counterpart and shows that phase information is transferred by projections of the charge density onto different modes. A direct consequence of the combination between the linear order with the coincidence measurement, is that the linear quantum diffraction scales as $\propto I_{p}^{1 / 2}$ with the interacting (signal) photons. The overall coincidence image scales as $\propto I_{p}^{3 / 2}$. This is beneficial for studying fragile complexes using weak fields, exploiting the $I_{p}^{1 / 2}$ scaling while enhancing the measured intensity according to $I_{p}^{3 / 2}$. Diffraction with classical source requires two 
interactions and therefore scales as $\propto I_{p}$ with the interacting photons, and an analog coincidence image scales as $\propto I_{p}{ }^{2}$. Entanglement based imaging is highly dependent on the degree of the initial correlations of the photons. Schmidt decomposition of the state of the field can be used to improve imperfect correlations by reweighting the Schmidt modes contributions. The apparent classical-like form of the coherent superposition in the Schmidt representation, where each mode carries a distinct spatial matter information, suggests experiments in which few Schmidt modes are measured at a time [141]. This allows the reweighting of high angular momentum modes available experimentally, and shown to have decreasing effect on the image otherwise.

Current and future challenges. Light manipulation techniques such as modal decomposition, while abundant in the optical regime, are not yet matured for x-rays. This challenge in the ability to measure each mode separately, can be addressed by directing the reweighting protocol to the post-processing stage. Decomposition of the image using a known data base of modes that correspond to the physical ones. Such protocol raises different set of challenges stemming from the sensitivity of decomposition techniques to noisy data. To that end, using methods to reduce the SNR beyond sub-shotnoise imaging can be extremely useful. By subtracting images generated using correlated photons, one of which transmitted through the image while the other does not, yet contains similar spatial noise [142].

X-ray diffraction has long been the work-horse of studies of small-scales of matter initially in equilibrium. Combining this with techniques used in modern spectroscopy [143], opens the possibility to follow and study complex dynamics in real time. Driving the sample into a non-stationary state using a series of pulses and delays, provides a set of useful dynamical control parameters. It allows to scan the potential landscape and extract information on the transition charge distribution such as the relaxation dynamics, providing a topic for future study. Applying such tools depends critically on the photon acquisition time versus the relevant dynamical scales of the system. Low photon fluxes desirable for fragile samples, limit the observable timescale.

Concluding remarks. Observing real time quantum dynamics of elementary chemical events, constitutes a long-standing scientific challenge. Possible applications range from studies of nonequilibrium quantum field theories to the engineering of quantum materials designed for specific tasks when driven. Recent technological advances in short wavelength ultrafast light sources, have revolutionized our observation window to the atomic scale [144]. With bright Free Electron Lasers and high harmonics tabletop sources, short time (femtosecond) and length (sub-nanometer) scales become accessible experimentally [145].

Scattering of quantum sources off matter carries phase information at odd orders in the charge distribution. These orders are associated with inversion symmetry breaking, carrying information directional motion and momentum of transient states. The field changes its state by the interaction, and phase is acquired. No photon is generated to linear order in the interaction. Homodyne diffraction of classical sources depends on even orders in the charge density and therefore lacks this information. These insights combined with the modern spectroscopic toolbox, open a new era in the study of quantum materials and nonequilibrium dynamics.

\section{Acknowledgments}

The support of the Chemical Sciences, Geosciences, and Biosciences Division, Office of Basic Energy Sciences, Office of Science, US Department of Energy Award No. DEFG0204ER15571, is gratefully acknowledged. SA was supported by this grant. SM was supported by the National Science Foundation (Grant No. CHE-1663822). We also wish to thank Noa Asban for the graphical illustrations. 


\section{Raman quantum eraser: the origin of quantum interferometer}

\author{
Marlan O Scully ${ }^{1,2,3}$, Girish S Agarwal', Tao Peng ${ }^{1}$, Alexei V \\ Sokolov ${ }^{1,2}$, Zhedong Zhang $^{1}$ and M Suhail Zubairy ${ }^{1}$ \\ ${ }^{1}$ Texas A\&M University, College Station, TX 77843, United \\ States of America \\ ${ }^{2}$ Baylor University, Waco, TX 76704, United States of \\ America \\ ${ }^{3}$ Princeton University, Princeton, NJ 08544, United States of \\ America
}

Introduction. In this article we review the 'Raman eraser' experiment where the which-path information is accessible to an observer and the 'erasure' of this information qualitatively changes the outcome, and give examples of technical applications, e.g. microscopy [146, 147].

We consider the interference between light scattered from atoms located at sites 1 and 2 as in figure 21(a) [146]. These atoms have three levels (see figure 21(b)), which are pumped from $c$ to $a$ by pulse, and interference fringes between the $\gamma$ photons emitted by atoms 1 and 2 are sought. An absence of interference between photons $\gamma_{1}$ and $\gamma_{2}$ in figure 21(a) is predicted when the states $b$ and $c$ are distinguishable. This is as expected, since an atom in the $b$ state has left information as to 'which path' the photon took. However, when we arrange to 'erase' this information via an appropriate photon correlation experiment the fringes can be made to reappear. That is, we may decide whether to emphasize wave like (interference) or particle-like (which path) behavior even after the emission is over.

Current and future challenges. The coincidence counting of photons can be effective in selecting useful signals through the photon-photon correlation as we shall see. This holds promise for using Raman photon pairs as a potential candidate for enhancing the utility of microscopy [148, 149].

Moreover, the traditional Raman spectroscopy, microscopy and lithography are bottlenecked by spatial resolution that is limited by the wavelength of light used to illuminate the sample. Although recently developed microscopy using tip-enhanced Raman may provide better spatial resolution, the possible mechanical damage to the materials will limit the application. However, by using the correlated photon pairs produced in Raman quantum eraser, the resolution of multiphoton quantum microscopy is substantially improved [148, 149]. Such a scheme also takes advantage of noninvasive detection. This can find immediate application in many biophysical studies, such as measuring the conformation of proteins and identifying the nucleotides in a DNA strand. One way of making such measurements involves markers, e.g. dye molecules or quantum dots, attached to two known points on protein and observing their fluorescence as they move apart. The correlated photons in Raman quantum eraser can be applied to measure the distance between markers, with improved resolution. Counting of

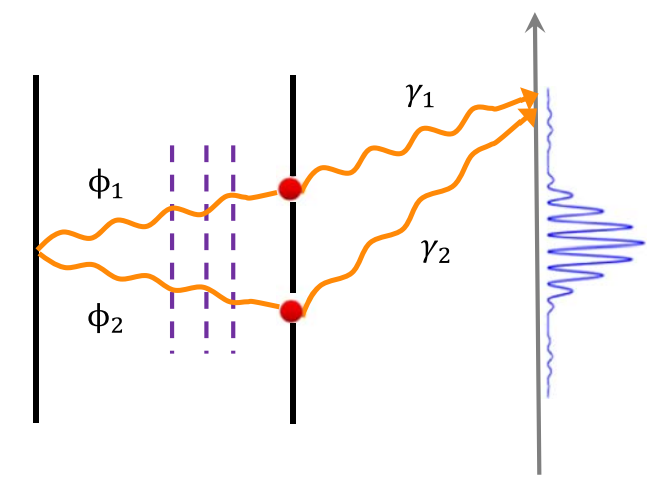

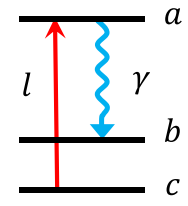

(b) (a)

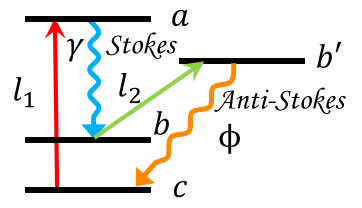

(c)
Figure 21. (a) Figure depicting light impinging from left on atoms at sites 1 and 2. Scattered photons $\gamma_{1}$ and $\gamma_{2}$ produce interference pattern on screen. (b) Three-level atoms excited by pulse $l_{1}$ from $c \rightarrow a$ and emit 'stokes' photons in $a \rightarrow b$ transition. (c) 'CARS' four-level system excited by pulse $l_{1}$ from $c \rightarrow a$ followed by emission of $\gamma$ Stokes photons in $a \rightarrow b$ transition. Second pulse $l_{2}$ takes atoms from $b \rightarrow b^{\prime}$. Decay from $b^{\prime} \rightarrow c$ results in emission of $\phi$ anti-Stokes photons.

photons can be effective in selecting the useful signal through photon-photon correlations.

\section{Advances in Science and Technology allow us to Meet the Challenges of better understanding quantum mechanics}

Quantum eraser with entangled light. A quantum eraser experiment very close to the original proposal [146] is illustrated in figure 22 [150]. An atom labeled by A or B is excited by a weak laser pulse. A pair of entangled quanta, 'photon' 1 and 'photon' 2, is then emitted from either atom A or atom B by atomic cascade decay. Photon 1, propagating to the right, is registered by detector $D_{0}$, which can be scanned by a step motor along it is $x$ axis for the observation of interference fringes. Photon 2, propagating to the left, is injected into a beam splitter. If the pair is generated by atom A, photon 2 will follow the A path meeting beam splitter A (BSA) with $50 \%$ chance of being reflected or transmitted. If the pair comes from atom B, photon 2 will follow the B path meeting beam splitter B (BSB) with $50 \%$ chance of being reflected or transmitted. In the case of the $50 \%$ chance of being transmitted at either BSA or BSB, photon 2 is detected by either detector $D_{3}$ or $D_{4}$. The registration of $D_{3}$ or $D_{4}$ provides the which-path information (path $\mathrm{A}$ or path $\mathrm{B}$ ) of photon 2 and this in turn provides the which-path information for photon 1 due to the entanglement nature of the two-photon state generated by atomic cascade decay. Given a reflection at either BSA or BSB photon 2 continues on its A or B path to meet another 50-50 beam splitter and then be detected by either detector $D_{1}$ or $D_{2}$ shown in figure 22. The triggering of detector $D_{1}$ or $D_{2}$ erases the which-path information of photon 

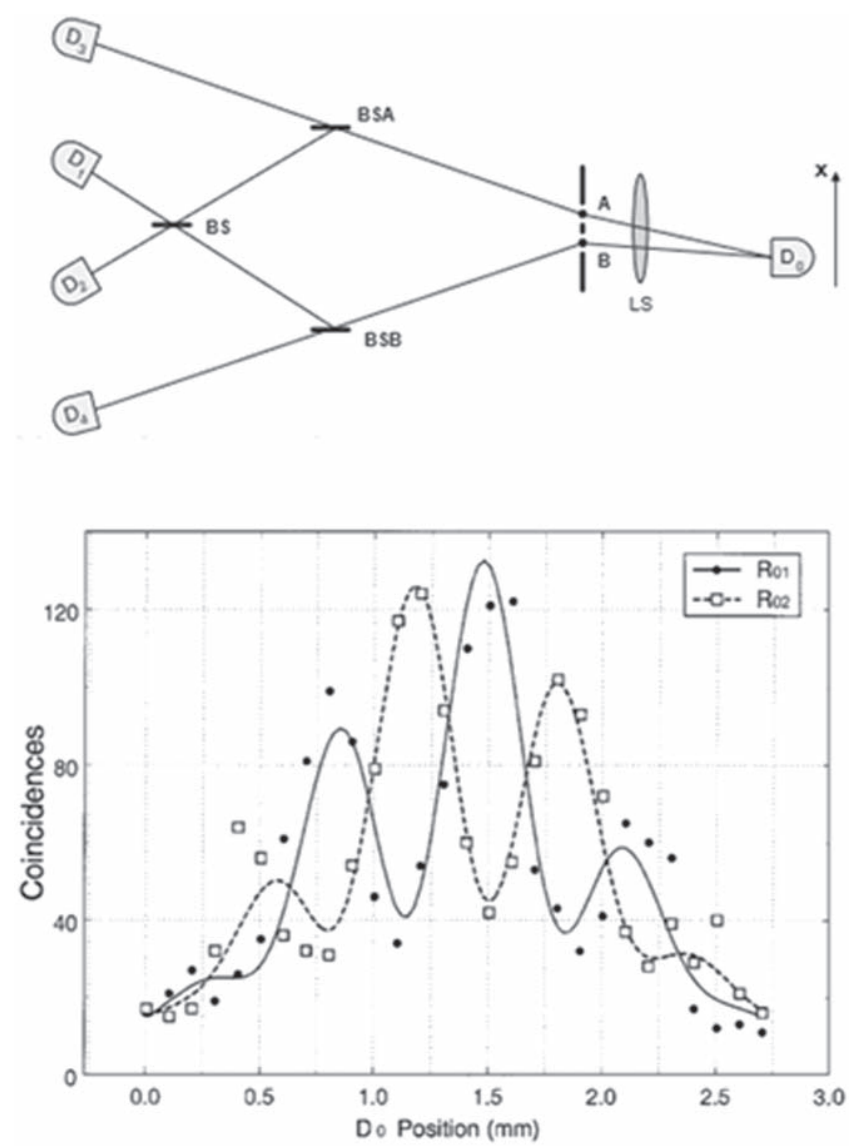

Figure 22. (Top) Proposed quantum eraser experiment. A pair of entangled photons is emitted from either atom A or atom B by atomic cascade decay. 'Clicks' at $\mathrm{D}_{3}$ or $\mathrm{D}_{4}$ provide the which-path information and clicks at $D_{1}$ or $D_{2}$ erase the which-path information. (Bottom) $R_{01}$ and $\mathrm{R}_{02}$ against the $\mathrm{x}$ coordinates of detector $\mathrm{D}_{0}$. Standard Young's double-split interference patterns are observed. Note the $\pi$ phase shift between $R_{01}$ and $R_{02}$. The solid line and dashed line are fits to the data based on equation (6) in [150].

1. Therefore, either the absence of the interference or its restoration can be arranged via an appropriately contrived photon correlation arrangement.

Raman eraser microscopy. Next we apply the Raman quantum eraser scheme to potentially improve microscopy [148]. We recall that light scattered from the two molecules of figure 23 generates a 'Young's' interference pattern at detector D1 given by $\cos ^{2}\left(k\left(r_{1}-r_{1}^{\prime}\right) / 2\right)=\cos ^{2}(k(d \sin \theta) / 2)$. The first interference minimum will occur when $k d \sin \theta_{m}=\pi$. Here $d$ is the distance between the marker molecules, $\theta_{m}$ is the angle between the normal to the line joining the two molecules and the vector pointing to the interference minimum and $k=2 \pi / \lambda$ as usual. However, in second order interferometry based on Raman quantum eraser the distance $d$ can be measured to a much greater precision, i.e. in the present scheme $d_{\text {min }} \sim \lambda / 13$ when the refractive indices $n_{2}$ and $n_{1}$ are properly chosen [148], see figure 24.

A major portion of the physics behind this enhanced resolution is understood by considering the original Raman quantum eraser configuration of figure 23 . Suppose that the

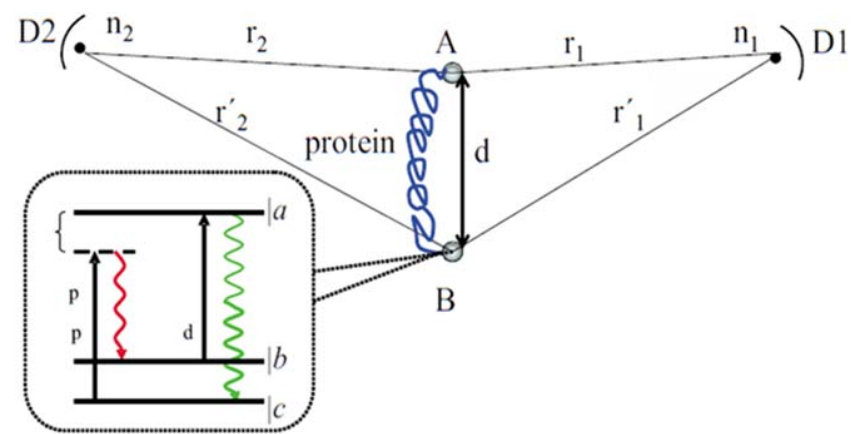

Figure 23. Marker molecules or quantum dots at $\mathrm{A}$ and $\mathrm{B}$ generate correlated pairs of photons by a Raman quantum eraser process in which the scatterers are pumped by a laser (frequency $v_{p}$ ) to a virtual level (dashed line) and spontaneously emit the first (Stokes) photon $(v)$. The molecule is then promoted from $b \rightarrow a$ by absorbing a drive laser photon (frequency $v_{\mathrm{d}}$ ) and spontaneously emits the second (anti-Stokes) photon $(\omega)$. The medium on the right (left) half plane has refractive index $n_{1}=1.9\left(n_{2}=2.1\right)$.

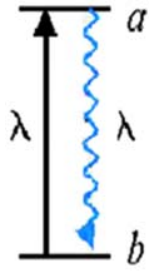

two level

(a)

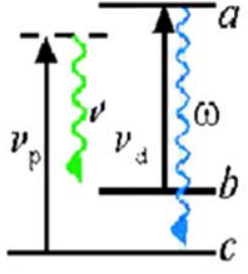

Raman (RED)

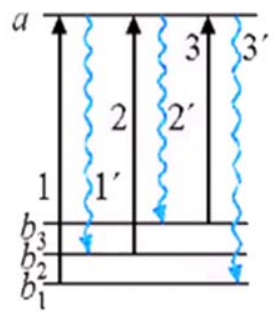

three-photon Raman

(c)
Figure 24. Various kinds of marker molecules. In every case $\lambda$ is the wavelength of the incident light, $\sin \theta=0.83$ and the refractive indices are $n_{1}=1.9$ while $n_{2}=n_{3}=2.1$. (a) Two level scheme yields classical, one photon resolution. (b) Raman eraser doublet (RED) scheme as discussed in the text. (c) Three photon Raman scheme that requires correlation between three detectors.

molecule is excited by a pulse having frequency $v_{p}$ and much later by a pulse at frequency $v_{d}$, then the spontaneous Stokes and anti-Stokes photons of figure 23 will be independent, and the photon-photon correlation function factorizes. We recall

$$
G_{v, \omega}^{(2)}=\left\langle\Psi\left|\hat{E}^{(-)}(1) \hat{E}^{(-)}(2) \hat{E}^{(+)}(2) \hat{E}^{(+)}(1)\right| \Psi\right\rangle,
$$

where $G_{v, \omega}^{(2)}=G_{v, \omega}^{(2)}\left(1,1^{\prime} ; 2,2^{\prime}\right), \hat{E}^{(+)}(1)$ is the positive frequency (annihilation) part of the electric field and $\hat{E}_{1}^{(-)}$is the corresponding negative frequency (creation) part; 1 and $1^{\prime}$ stand for $r_{1}, t_{1}$ and $r_{1}^{\prime}, t_{1}^{\prime}$, etc as in figure 23. The times $t_{i}$ are controlled by e.g. shutters. The frequency of the first (Stokes) photon is $v=v_{p}+v_{d}-\omega_{a c}$ and the second (antiStokes) is $\omega=\omega_{a c}$. If the two emitted photons are well separated in time, then the twophoton state can be factorized as a sum of products of one photon Stokes and anti-Stokes states from molecules at A and B, that is

$$
|\Psi\rangle=\frac{1}{\sqrt{2}}\left[\left|v_{A}\right| \omega_{A}+\left|v_{B}\right| \omega_{B}\right]
$$

Now we take advantage of the fact that the first and second photons have different frequencies and can therefore 
be separated by, for example, a dichroic mirror. Thus, we can arrange that the $v$ and $\omega$ photons go to, say, D1 and D2 respectively. Equation (4) can then be written as $G_{v, \omega}^{(2)}=\left|\varepsilon_{\omega A}(2) \varepsilon_{v A}(1)+\varepsilon_{\omega B}(2) \varepsilon_{v B}(1)\right|^{2} \quad$ using $\quad E_{\eta A}(i)=$ $0\left|\hat{E}^{(+)}(i)\right| \eta_{A} \propto e^{-i \eta \tau_{i}}$ and $\mathcal{E}_{\eta B}(i)=0\left|\hat{E}^{(+)}(i)\right| \eta_{B} \propto e^{-i \eta \tau_{i}^{\prime}}$ where $\tau_{i}=t_{i}-n_{i} r_{i} / c$ are the retarded times, $r_{i}\left(r_{i}^{\prime}\right)$ are the distances between molecule $\mathrm{A}(\mathrm{B})$ and the $i$ th detectors and $n_{i}$ are the refractive indices with $\eta=v, \omega$ and $i=1,2$. It follows that

$$
G_{v, \omega}^{(2)}=C \cos ^{2}\left[\frac{1}{2}\left(\kappa_{v}+\kappa_{\omega}\right) d \sin \theta\right],
$$

where $\kappa_{v}=\frac{n_{1} v}{c}, \quad \kappa_{\omega}=n_{1} \omega / c$ and we have used $r_{1}^{\prime}-r_{1}=$ $r_{2}^{\prime}-r_{2}=d \sin \theta$. Here the detection times $t_{1}^{\prime}=t_{1}$ and $t_{2}^{\prime}=t_{2}$. Hence, the interference pattern observed by moving detector 1 (and requiring a correlation with detector 2) is now governed by $\kappa_{v}+\kappa_{\omega} \simeq 2 k$, i.e. the effective radiation wavelength is now $\lambda / 2$.

The quantum eraser scheme [146] used a Raman doublet configuration and sorted between fringes and anti-fringes via a cavity and shutter arrangement. The two-photon spectroscopy paper with Rathe [151] used the same detection scheme as in the present paper but with cascade radiation. Subensemble sorting with such a detection scheme is accomplished by tailoring the signal as seen by $\mathrm{D} 2$ relative to D1; see e.g. section 21.2.1 of [152]. A similar scheme can be used with incoherently excited two-level atoms [153]. Furthermore in the experiment of [154] they say that: 'One has to 'erase' the first-order interference by reinforcing an experimental condition: $\delta \theta>\lambda / b$, where $\delta \theta$ is the divergence of the light and $b$ is the distance between the two slits'. It is also interesting to note that in the case of the three photon Raman scheme of figure 24(c), we must end in level $b_{1}$ (register a D2 count) in order to assure that we have erased which-way information.

Concluding remarks. We have presented the Raman eraser in connection with Raman microscopy. The quantum interference via photon pathways is shown to play an important role in improving the spatial resolution. This new spectroscopic technique using the quantum nature of photons holds promise for a powerful tool for resolving structural information about materials. The Raman-eraser scheme can be extended to multiple dimensions by subjecting the molecules to sequence of pulses. This may lead to a new photon-coincidence counting spectroscopy. This technique contains the information of higher-order correlations between molecules, and may help in resolving the quantum fluctuation effects. The material presented in this short note is a small example of new techniques we are pursuing.

\section{Acknowledgments}

We gratefully acknowledge the support of grants AFOSR Award FA-9550-18-1-0141, ONR (Award N00014-16-13054 and N00014-16-1-2578) and Robert A Welch Foundation (Award A-1261, A-1547 and A-1943-20180324), and the grant King Abdulaziz City for Science and Technology (KACST). MOS thanks Prof Shaul Mukamel for many stimulated discussions and ideas. 


\section{Hanbury Brown and Twiss interferometry at XFEL sources}

\section{Ivan A Vartanyants}

DESY, Germany; NRNU 'MEPhI', Russia

Status. In their pioneering experiments, Hanbury Brown and Twiss (HBT) demonstrated [155] that fundamental information on the statistics of light sources can be acquired by coincidence detection of photons. This intensity interferometry was originally designed to determine the size of stars, but subsequently raised fundamental questions in the field of optical coherence and gave birth to the field of quantum optics [156].

The core idea of a HBT experiment is to determine the normalized second-order intensity correlation function $g^{(2)}\left(\boldsymbol{r}_{1}, \boldsymbol{r}_{2}\right)=\left\langle I\left(\boldsymbol{r}_{1}\right) \cdot I\left(\boldsymbol{r}_{2}\right)\right\rangle /\left[\left\langle I\left(\boldsymbol{r}_{1}\right)\right\rangle\left\langle I\left(\boldsymbol{r}_{2}\right)\right\rangle\right]$ by measuring the coincident intensities $I\left(\boldsymbol{r}_{1}\right)$ and $I\left(\boldsymbol{r}_{2}\right)$ of two detectors at separated positions $\boldsymbol{r}_{1}$ and $\boldsymbol{r}_{2}$. Averaging is done over a large number of realizations of the wave fields.

Importantly, such measurements can distinguish between the sources of chaotic and coherent light. Glauber has formulated [156] that the source to be coherent it should be coherent at all orders of correlation function. That may lead to situation when the source is coherent at its first-order but behaves as a chaotic source at its second-order.

Chaotic light can be completely described in the frame of Gaussian statistics and its statistical properties are determined by the first-order correlation function $g^{(1)}\left(\boldsymbol{r}_{1}, \boldsymbol{r}_{2}\right)$ that is defined as correlation of the amplitudes of the fields. The secondorder intensity correlation function then reduces to $g^{(2)}\left(\boldsymbol{r}_{1}, \boldsymbol{r}_{2}\right)=1+\zeta_{2}\left(D_{\omega}\right) \cdot\left|g^{(1)}\left(\boldsymbol{r}_{1}, \boldsymbol{r}_{2}\right)\right|^{2}$, where the contrast $\zeta_{2}\left(D_{\omega}\right) \sim 1 / D_{\omega} T$ is inversely proportional to the bandwidth $D_{\omega}$ and radiation pulse duration $T$. Importantly, by analyzing the contrast as a function of the bandwidth from HBT interferometry it is possible to determine an average pulse duration.

A defining feature of any laser source is its high degree of coherence. It was recently demonstrated [157, 158] that XFELs based on the self-amplified spontaneous emission (SASE) generation process have a high degree of the firstorder correlation function $g^{(1)}\left(\boldsymbol{r}_{1}, \boldsymbol{r}_{2}\right)$ or transverse coherence, but low temporal coherence. To get a more detailed picture of the statistical properties of these sources, higher-order correlations of the XFEL wave fields must be studied. Measurements of second- and higher-order intensity correlation functions were recently performed at different XFEL sources [159-162] by implementing HBT interferometry.

Our measurements of the first-order correlation functions at different SASE based XFEL sources [157, 158] have shown that they behave as a highly coherent sources with a global degree of the first-order spatial coherence of about $80 \%$. A high value of the degeneracy parameter (on the order of $10^{9}$ ), which is the number of photons in a single coherent mode was determined at these sources. This value is by many orders of magnitude higher than that of any synchrotron source. (a)

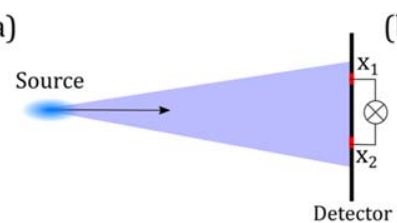

(b) (c)

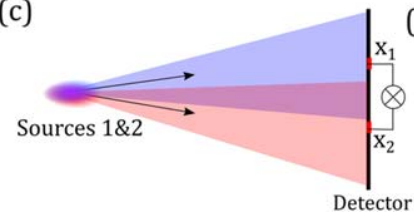

Source 1

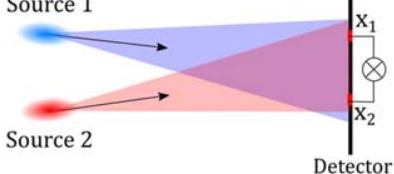

(d)

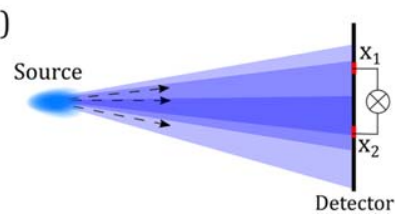

Figure 25. Intensity correlations for different configuration of chaotic extended sources. (a) Single source. (b) Two sources separated from each other. (c) Single source radiating in multiple angular positions. (d) Single source with the angular jitter (from [160]). Reprinted figure with permission from [160], Copyright 2017 by the American Physical Society.

Measurements of the second-order correlation function at different XFEL sources [159-162] has revealed that radiation is strongly inhomogeneous and its behavior could not be explained by radiation coming from a single distant extended source (see figure 25). We developed advanced theoretical models, which included a multiple-beam model and external positional jitter, to account for these effects [160]. The obtained results demonstrate that the HBT interferometry is a very sensitive method for the XFEL beam statistical characterization.

An interesting development of HBT interferometry was presented in recent work [161]. Here, for the first time, HBT interferometry was performed in diffraction mode at an XFEL source. It allowed to determine the XFEL statistical properties directly from the Bragg peaks originating from the colloidal crystal. This approach is different from the traditional one when HBT interferometry is performed in the direct beam without a sample. Our analysis has also allowed to determine an average pulse duration that was on the order of ten femtoseconds for the monochromatic beam, which was significantly shorter than expected from the electron bunch measurements.

At the same time, measurements of the higher-order correlation functions suggest that SASE XFELs are essentially chaotic sources obeying Gaussian statistics. This raised the fundamental question, whether there are coherent XFEL sources satisfying Glaubers definition of coherence. A definitive answer to that question was given in HBT interferometry measurements performed at the externally seeded XFEL source FERMI [162].

A combination of HBT interferometry and spectral measurements demonstrated that a seeded XFEL is fundamentally different in its statistical properties from a SASEbased XFEL [162]. These measurements are a decisive step forward in understanding the basic properties of XFELs. While SASE FELs behave statistically as chaotic sources, the seeded FERMI XFEL is equivalent in its statistical properties to a coherent laser in the definition of Glauber with its secondorder $g^{(2)}$-function close to one. Importantly, the degeneracy parameter for the seeded FEL FERMI reaches a number as 


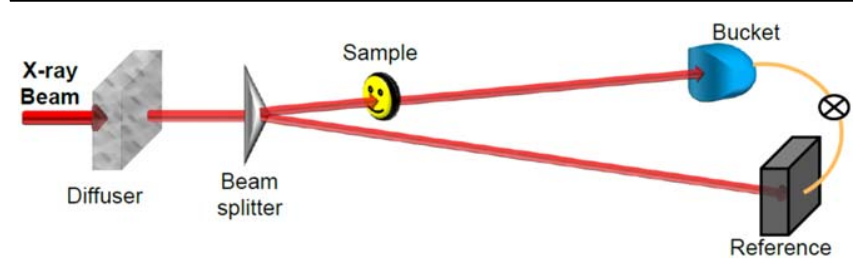

Figure 26. Schematic layout of a ghost imaging experiment.

high as $10^{11}-10^{12}$. This is by two orders of magnitude higher than in the case of SASE XFELs, where a monochromator has to be used to pass a single longitudinal mode.

Current and future challenges. Within quantum optics the subject of quantum imaging, based on utilizing correlation techniques, is presently evolving particularly rapidly [163]. Employing techniques of quantum imaging may lead to an enhanced resolution that can, in principle, overcome Abbe's fundamental limit of classical optics [164]. One of the schemes proposed and developed in the domain of quantum imaging is the so-called GI [165]. The idea is based on parallel measurement of two correlated beams, where one beam passes through the object and the transmitted light is detected using a bucket detector, whereas the other beam is freely propagating and recorded using a pixelized detector (see figure 26). Coincidence measurements are then performed between each pixel of the pixelized and bucket detectors, so that the object is finally reconstructed by coincident detection of the two photons at both detectors.

Initially it was assumed that GI needs quantum correlations between the two beams, but later it was realized that measurements can also be performed using classically correlated coherent light beams. One of the appealing features of GI is that by varying the ratio of the photon flux in the two arms, one can substantially lower the radiation dose absorbed by the sample while increasing the intensity of the other beam that does not impinge on the sample. As such, radiation damage, that is the most severe factor limiting the resolution in biological imaging, may be alleviated. The first realization of the classical GI at FEL sources is presented in a recent work [166].

Concluding remarks. An interesting future application of the intensity interferometry methods would be the study of seeded FELs. An intriguing question is whether seeded FELs are fully coherent sources in all orders, as described by Glauber [156], and, in this way, are equivalent to conventional single mode lasers, or if they obey Gaussian statistics like SASE FELs. We could also foresee that intensity correlation analysis might be applied to study the dynamics of ultrafast processes at FELs. Even more thrilling is that our measurements demonstrated a high degree of spatial coherence of the FEL radiation, which could potentially lead to a completely new avenue in the field of quantum optics. This could lead to quantum optics experiments such as the exploration of nonclassical states of light, superresolution and quantum imaging experiments [164], or GI experiments [166] at the FEL sources. This in turn is an important prerequisite for a new class of light phase-sensitive experiments such as coherent and phase control interferometry with attosecond precision.

\section{Acknowledgments}

I would like to acknowledge E Weckert who supported our research on intensity interferometry at DESY, W Wurth and his group in collaboration with whom most of experiments were performed, as well as A Singer, O Gorobtsov, G Mercurio, Y Y Kim, L Gelicio, L Giannessi, K Prince, and many others for their dedicated work on the project. I would like specially to thank teams at different XFEL sources: FLASH, LCLS, FERMI for their strong contribution for the success of the measurements performed at XFELs. The support of Helmholtz Associations Initiative and Networking Fund and Russian Science Foundation (Grant No. HRSF-0002/18-4106001) is gratefully acknowledged. 


\section{The true colors of quantum light}

\author{
Elena del Valle ${ }^{1}$ and Fabrice $P$ Laussy ${ }^{1,2,3}$ \\ ${ }^{1}$ Universidad Autónoma de Madrid, Spain \\ ${ }^{2}$ University of Wolverhampton, United Kingdom \\ ${ }^{3}$ Russian Quantum Center, Russia
}

Status. The idea of adding the frequency variable, i.e. the color, to photon-correlation measurements was initiated in 1980 by Aspect et al in an experiment on resonance fluorescence [167]. Placing spectrally-sensitive devices in front of detectors showed that the two sidebands of the spectral shape-the 'Mollow triplet'-emitted photons in a well-defined time order. At first, these results were theoretically predicted by Apanasevich and Kilin [168] and described by Reynaud, Dalibard and Cohen-Tannoudji [167], using specific methods for this particular system. A more general, platform-independent theory of frequency filtered $N$-photon correlations, extending the 'physical spectrum' of Eberly and Wódkiewicz [169], was developed during the 80 s by Knöll, Weber, Nienhuis, Vogel, Welsch and Cresser, among others [170, 171]. The measured field at frequency $\omega, A_{\omega}$, is there described as a convolution of the single-mode naked field, $a$, with the transmission response function of the spectral apparatus (detector of central frequency $\omega$ and linewidth $\Gamma$ ). This defines time- and frequency-resolved $N$-photon coherence functions, $\left\langle A_{\omega_{1}}^{+}\left(t_{1}\right) \ldots A_{\omega_{N}}^{+}\left(t_{N}\right) A_{\omega_{N}}\left(t_{N}\right) \ldots A_{\omega 1}\left(t_{1}\right)\right\rangle$, that describe correlations between photons detected at their respective specific times $t_{i}$ and frequencies $\omega_{i}$. These generalized versions of the color-blind Glauber coherence functions, $G^{(N)}\left(t_{1}, \ldots, t_{N}\right)$, provide the full information one can have on photon correlations, and are thus both of intrinsic and fundamental interest. Since time and frequency are conjugate variables, their combined knowledge is constrained. This is assured by the detector linewidth, which yields a new degree of freedom to tailor photon correlations, with, as limiting cases, the textbook results: at large $\Gamma$, the standard $G^{(N)}$ are recovered. At small $\Gamma$, the HBT boson statistics is recovered, for instance, at thermal equilibrium or under incoherent excitation, $G^{(N)} \sim N$ ! when photons are indistinguishable $\left(\omega_{1}=\omega_{2}=\ldots=\omega_{N}\right)$ and correlations are lost if photons are distinguishable. The multiplicity of photons also requires a careful time $(T)$ and normal $(:)$ ordering of the field operators, of the form $\left\langle: T\left[a^{+}\left(t_{1}\right) \ldots a^{+}\left(t_{N}\right)\right] T\left[a\left(t_{N}\right) \ldots a\left(t_{1}\right)\right]:\right\rangle$ that need to be computed and integrated in all time variables, in all possible time orderings. This makes the frequencyresolved photon-correlation a very complicated quantity to compute even if making use of recurrence relations [172] or dedicated diagrammatic techniques [173]. Already for the case of intensity-intensity correlations $(N=2)$, it involves the integration of 12 independent correlators (with different time orders), each requiring recursive application of the quantum regression formula. In 2012, we developed a theoretical method (the sensors method) [172] that is both conceptually and technically simpler than the physical spectrum. As sketched in figure 27, it consists in including
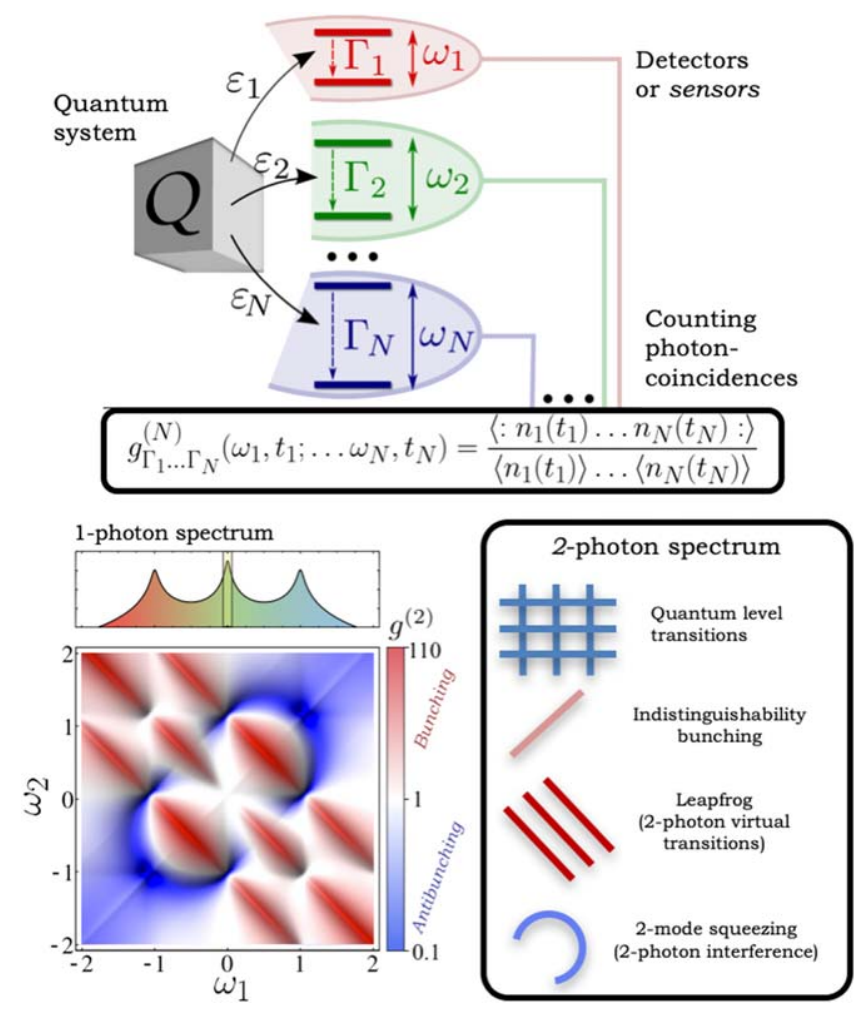

Figure 27. Scheme of the sensors method with an example of a 2-photon spectrum (2PS) with signature features.

frequency-sensitive detectors in the dynamics, with vanishing coupling $\varepsilon \rightarrow 0$ so as not to affect the system itself. Another option is Gardiner's cascaded formalism, that ensures unidirectional coupling even for non-negligible populations (although the computational gain is not so good, this enables complementary approaches such as frequency-resolved Monte Carlo simulations [174]). We have shown that correlations between sensors in the limit $\varepsilon \rightarrow 0$ are mathematically equal to the cumbersome integral expressions (and to the cascaded formalism), thereby providing exact results and with no restriction, in a completely general fashion (i.e. time-dependent frequencyresolved correlations up to any photon number for any quantum open system). Since the sensors can in most cases be modeled by two-level systems, the technique is numerically very efficient (for autocorrelations, truncated harmonic modes can be used instead, up to $N$ excitations for $\mathrm{N}$-photons correlations). This allowed us to compute frequency correlations not exclusively between the spectral features (the peaks, i.e. the central and satellites peaks in the case of the Mollow triplet) but between photons of arbitrary frequencies, and without approximations in the description [175] (unlike the earlier treatments of resonance fluorescence). This is an extremely rewarding endeavor since it turns out that the strongest and most interesting photon correlations hide away from the spectral features and actually occur in previously unsuspected frequency windows. Spanning over all possible frequencies unveils a rich landscape, that we call the $N$-photon correlation spectrum. In the simplest case of two-photon coincidences 
(zero delay between detected photons), this yields a 2D map [175], an example of which is shown in figure 27 for resonance fluorescence. There are clear features, which can all be accounted for: the horizontal and vertical patterns stem from the expected correlations between dressed states, that define the quantum energy levels of the system. These are the ones that had been focused on since the 80s. More important for quantum applications, the antidiagonal lines correspond to two-photon transitions (we call them leapfrog transitions as they jump over intermediate rungs of the Mollow ladder of dressed states). They happen through virtual photons and the emission is thus strongly quantumcorrelated, with classical-inequality violation [176]. Finally, the circles of antibunching originate from two-mode squeezing. Once identified, such correlations can be optimized by frequency filtering [177].

Although filtered $g^{(2)}$ have been routinely obtained in the laboratory for particular frequencies since the $80 \mathrm{~s}$, it is only recently that the full structure of two-photon correlations has been experimentally observed. This was first reported for the frequency-resolved testbed-resonance fluorescence [178]and shortly after for polaritons [179], finding spectacular agreement with the theory, confirming all the features in figure 27, including classical-inequality violation. These experiments put to rest a controversy about the actual existence of leapfrog correlations [173], as discussed extensively in [175].

Current and future challenges. The knowledge of such rich and strong quantum correlations calls for their exploitation in a variety of quantum applications. The phase-space is huge with $N$ photons [180] and detailed investigations should be undertaken to explore the possibilities of multi-photon correlations. The first concept of a device that follows from the frequency-resolved paradigm is indeed the most straightforward counterpart of the single-photon source, where instead of emitting a single photon, one emits $N$ instead (with $N$ any integer). A CW $N$-photon emitter [181] can be realized by using the signature strategy of cavity QED: Purcell-enhancing the emission with a cavity. For strongenough coupling, the transition can become the dominant channel of emission. Further designs should be developed to realize other types as well as more useful devices, such as pulsed $N$-photon emission or heralded $N$-photon sources. The latter could be based on non-degenerate leapfrog transitions, with one photon (the heralding) emitted at a different frequency from the others (the heralded). This would allow to scale-up the number of generated photons by multiplexing techniques. The generation of very-large (hundreds or more) photons in a deterministic Fock state, required in LOQC applications, would thus be pretty much settled. Other types of quantum correlations and effects arising from their interferences should also be developed, with likely improvements already at the bi-photon level, since two-mode squeezing can similarly be tuned and optimized. Generalization to higher-order $N$ mode squeezing with no principle restriction on $N$ are particularly attractive. This brings in direct sight a wide range of strongly-correlated quantum light for applications in quantum spectroscopy [182]. Finally, since a sensor is an exponentially decaying harmonic mode, it has a Lorentzian spectral profile, which corresponds to an interferometer of the Fabry-Pérot type (a dielectric layer) or a weakly-coupled cavity or waveguide. While this describes a fair share of the experimental situations, it is conceivable that other types of popular filters (such as square or Gaussian) would further expand the enhancement and tailoring of photon correlations. This however requires a more involved treatment if not reverting to the integral form, namely, using a set of sensors with tailored frequencies and couplings so that, collectively, they match the specific response function. Much theoretical studies therefore remain to explore these and similar prospects.

Advances in science and technology to meet challenges.

While the emergence of rich and strong quantum correlations from the process of filtering have been experimentally confirmed in spontaneous emission, including the leapfrog transitions [178], there has been no direct exploitation in a device-oriented context so far, which would clearly impulse tremendously the field. Even the highly-investigated $N$-photon emitter, which theoretical estimates place well within the state of the art [183], remains to be realized in the laboratory. Since this would produce no less than a new type of quantum light, where each photon is substituted by a bundle of $N$ photons, with multiple applications from quantum information processing to quantum spectroscopy, the incentives should be huge to deliver such a device. This appears to be essentially a technological problem, namely, that of bringing together the Mollow physics (driving a two-level system) and the Jaynes-Cummings physics (coupling a two-level system to a cavity) which both imply in the real world their respective tricks and optimized samples. Problems include those of attenuating a strong laser driving a two-level system without contaminating the cavity that collects the quantum signal. While clearly a nontrivial configuration, this seems nothing out of reach of resourceful and ingenious experimentalists. Demonstration of essentially pure 2- or even 3 -photon emitters as well as proof of principle demonstration of higher $N$-photon emission are within reach of the current figures of merits of most groups working on light-matter coupling in a variety of platforms, from semiconductor to atomic systems passing by superconducting qubits and trapped ions. This would already render useful services in terms of applications. It is likely that other concepts such as heralded $\mathrm{N}$-photon sources will make higher demands on the experimental configuration, such as using several cavities (one for the heralding photon, the other for the heralded) with stronger coupling, to be delivered by progress in material science and better technology. But their basic design still remains, at this stage, a challenge for theorists.

Concluding remarks. In summary, frequency-filtered photon correlations, or adding the color degree of freedom in quantum optics, not only provides clear and new insights into the quantum dynamics and level structure of an emitter, it also enables one to access, tailor and optimize different regimes of operation, simply by tuning the frequency and 
bandwidth of detection. One can easily switch in this way between different types of quantum mission, from singlephoton to thermal, $N$-photon, entangled or squeezed, using the same source.

\section{Acknowledgments}

EdV acknowledges support from MINECO (FIS2015-64951$\mathrm{R}$, Ramón y Cajal). 


\section{ORCID iDs}

Shaul Mukamel (i) https://orcid.org/0000-0002-6015-3135 Wolfgang Schleich (ib https://orcid.org/0000-0002-9693-8882 Marco Bellini (ib https://orcid.org/0000-0001-7352-1366 Alessandro Zavatta (iD https://orcid.org/0000-0002-1359-7956 Gerd Leuchs (iD https://orcid.org/0000-0003-1967-2766 André Stefanov (i) https://orcid.org/0000-0002-5588-7986 Marco Barbieri (ib https://orcid.org/0000-0003-2057-9104 Konstantin Dorfman (1) https://orcid.org/0000-0001-9963-0878 Vahid Sandoghdar (ib https://orcid.org/0000-0003-2594-4801 Michael Raymer (ib https://orcid.org/0000-0002-1661-7182 Bao-Sen Shi $\mathbb{1}$ https://orcid.org/0000-0002-5965-5365 Zhe-Dong Zhang (i) https://orcid.org/0000-0002-0305-6064 Ivan A Vartanyants (iD https://orcid.org/0000-0002-0340-8234 Elena del Valle (i) https://orcid.org/0000-0002-0221-282X

\section{References}

[1] Mack H and Schleich W P 2008 A photon viewed from Wigner phase space Opt. Photonics News Trends 3 29-36

[2] Schleich W P 2001 Quantum Optics in Phase Space (New York: Wiley)

[3] Vogel K, Akulin V M and Schleich W P 1993 Quantum state engineering of the radiation field Phys. Rev. Lett. 71 1816-9

[4] Glauber R J 2007 Quantum Theory of Optical Coherence: Selected Papers and Lectures (New York: Wiley)

[5] Lamb W E, Schleich W P, Scully M O and Townes C H 1999 Laser physics: quantum controversy in action Rev. Mod. Phys. 71 S263-73

[6] Bimbard E, Jain N, MacRae A and Lvovsky A I 2010 Quantum-optical state engineering up to the two-photon level Nat. Photonics 4 243-7

[7] Cooper M, Wright L J, Söller C and Smith B J 2013 Experimental generation of multi-photon Fock states Opt. Express 21 5309-17

[8] Haroche S 2013 Nobel lecture: controlling photons in a box and exploring the quantum to classical boundary Rev. Mod. Phys. 85 1083-102

[9] Ourjoumtsev A, Tualle-Brouri R, Laurat J and Grangier P 2006 Generating optical Schrödinger kittens for quantum information processing Science 312 83-6

[10] Schnabel R 2017 Squeezed states of light and their applications in laser interferometers Phys. Rep. 684 1-51

[11] Kira M, Koch S W, Smith R P, Hunter A E and Cundiff S T 2011 Quantum spectroscopy with Schrödinger-cat states Nat. Phys. 7 799-804

[12] Doherty M W, Manson N B, Delaney P, Jelezko F, Wrachtrup J and Hollenberg L C L 2013 The nitrogenvacancy colour centre in diamond Phys. Rep. 528 1-45

[13] Laibacher S and Tamma V 2015 From the physics to the computational complexity of multiboson correlation interference Phys. Rev. Lett. 115243605

[14] Wang H et al 2017 High-efficiency multiphoton boson sampling Nat. Photonics 11 361-5

[15] Jeong H, Zavatta A, Kang M, Lee S, Costanzo L S, Grandi S, Ralph T C and Bellini M 2014 Generation of hybrid entanglement of light Nat. Photonics 8 564-9

[16] Costanzo L S, Coelho A S, Pellegrino D, Mendes M S, Acioli L, Cassemiro K N, Felinto D, Zavatta A and Bellini M 2016 Zero-area single-photon pulses Phys. Rev. Lett. 116023602

[17] Bellini M and Zavatta A 2010 Manipulating light states by single-photon addition and subtraction Prog. Opt. 55 41-83
[18] Biagi N, Costanzo L S, Bellini M and Zavatta A 2020 Entangling macroscopic light states by delocalized photon addition Phys. Rev. Lett. 124033604

[19] Lamine B, Fabre C and Treps N 2008 Quantum improvement of time transfer between remote clocks Phys. Rev. Lett. 101 123601

[20] Mosley P J, Lundeen J S, Smith B J, Wasylczyk P, U'Ren A B, Silberhorn C and Walmsley I A 2008 Heralded generation of ultrafast single photons in pure quantum states Phys. Rev. Lett. 100133601

[21] Polycarpou C, Cassemiro K N, Venturi G, Zavatta A and Bellini M 2012 Adaptive detection of arbitrarily-shaped ultrashort quantum light states Phys. Rev. Lett. 109053602

[22] Ansari V, Donohue J M, Brecht B and Silberhorn C 2018 Tailoring nonlinear processes for quantum optics with pulsed temporal-mode encodings Optica 5 534-50

[23] Davis A O C, Thiel V, Karpiński M and Smith B J 2018 Measuring the single-photon temporal-spectral wave function Phys. Rev. Lett. 121083602

[24] Law K C, Walmsley I A and Eberly J H 2000 Continuous frequency entanglement: effective finite hilbert space and entropy control Phys. Rev. Lett. 845304

[25] Gatti A, Corti T, Brambilla E and Horoshko D B 2012 Dimensionality of the spatiotemporal entanglement of parametric down-conversion photon pairs Phys. Rev. A 86 053803

[26] Brecht B, Reddy D V, Silberhorn C and Raymer M G 2015 Photon temporal modes: a complete framework for quantum information science Phys. Rev. X 5041017

[27] Lukens J M and Lougovski P 2017 Frequency-encoded photonic qubits for scalable quantum information processing Optica 4 8-16

[28] Dorfman K E, Schlawin F and Mukamel S 2016 Nonlinear optical signals and spectroscopy with quantum light Rev. Mod. Phys. 88045008

[29] Ra Y-S, Jacquard C, Dufour A, Fabre C and Treps N 2017 Tomography of mode-tunable coherent single-photon subtractor Phys. Rev. X 7031012

[30] Zavatta A, Artoni M and La Rocca G C 2019 Engineering of heralded narrowband color-entangled states Phys. Rev. A 99 $031802(\mathrm{R})$

[31] Einstein A, Podolsky B and Rosen N 1935 Can quantummechanical description of physical reality be considered complete? Phys. Rev. 47 777-80

[32] Woodward P M 1953 Probability and Information Theory with Applications to Radar (New York: McGraw-Hill)

[33] Paye J 1991 The chronocyclic representation of ultrashort light pulses IEEE J. Quantum Electron. 28 2262-72

[34] Beck M, Raymer M G, Walmsley I and Wong V 1993 Chronocyclic tomography for measuring the amplitude and phase structure of optical pulses Opt. Lett. 18 2041-3

[35] Trebino R, DeLong K W, Fittinghoff D N, Sweetser J N, Krumbügel M A, Richman B A and Kane D J 1997 Measuring ultrashort laser pulses in the time-frequency domain using frequency-resolved optical gating Rev. Sci. Instrum. 68 3277-95

[36] Anashkina A E, Ginzburg V N, Kochetkov A A, Yakovlev I V, Kim A V and Khazanov E A 2016 Singleshot laser pulse reconstruction based on self-phase modulated spectra measurements Sci. Rep. 633749

[37] Reid M D 1989 Demonstration of the Einstein-PodolskyRosen paradox using nondegenerate parametric amplification Phys. Rev. A 40 913-23

[38] Ou Z Y, Pereira S F, Kimble H J and Peng K C 1992 Phys. Rev. Lett. 68 3663-6

[39] Silberhorn C, Lam P K, Weiß O, König F, Korolkova N and Leuchs G 2001 Generation of continuous variable EinsteinPodolsky-Rosen entanglement via the Kerr nonlinearity in an optical fiber Phys. Rev. Lett. 86 4267-70 
[40] Klyshko D 1989 The Einstein-Podolsky-Rosen paradox for energy-time variables Sov. Phys. Usp. 32 555-63

[41] Dorfman K E, Schlawin F and Mukamel S 2014 Stimulated Raman spectroscopy with entangled light: enhanced resolution and pathway selection J. Phys. Chem. Lett. 5 2843-9

[42] Donohue J M, Ansari V, Řeháček J, Hradil Z, Stoklasa B, Paúr M, Sánchez-Soto L L and Silberhorn C 2018 Quantumlimited time-frequency estimation through mode-selective photon measurement Phys. Rev. Lett. 121090501

[43] Leuchs G, Dong R and Sych D 2009 Triplet-like correlation symmetry of continuous variable entangled states New J. Phys. 11113040

[44] Horodecki M and Horodecki P 1999 Reduction criterion of separability and limits for a class of distillation protocols Phys. Rev. A 59 4206-16

[45] Howell J C, Bennink R S, Bentley S J and Boyd R 2004 Realization of the Einstein-Podolsky-Rosen paradox using momentum- and position-entangled photons from spontaneous parametric down conversion Phys. Rev. Lett. 92 210403

[46] Rarity J, Tapster P and Jakeman E 1990 Two-photon interference in a Mach-Zehnder interferometer Phys. Rev. Lett. 65 1348-51

[47] Olislager L, Cussey J, Nguyen A, Emplit P, Massar S, Merolla J-M and Huy K 2010 Frequency-bin entangled photons Phys. Rev. A 82 1-7

[48] Bernhard C, Bessire B, Feurer T and Stefanov A 2013 Shaping frequency-entangled qudits Phys. Rev. A 88032322

[49] Nasr M, Saleh B, Sergienko A and Teich M 2003 Demonstration of dispersion-canceled quantum-optical coherence tomography Phys. Rev. Lett. 91 8-11

[50] Brendel J, Zbinden H and Gisin N 1998 Measurement of chromatic dispersion in optical fibers using pairs of correlated photons Opt. Commun. 151 35-9

[51] Stefanov A 2009 Frequency comparison using energy-time entangled photons Frequency Standards and Metrology (Singapore: World Scientific) pp 559-63

[52] Valencia A, Scarcelli G and Shih Y 2004 Distant clock synchronization using entangled photon pairs Appl. Phys. Lett. 85 2655-7

[53] Lerch S 2016 Energy-time entangled two-photon states for quantum information and nonlinear spectroscopy $P h D$ Thesis

[54] Dayan B, Pe'er A, Friesem A A and Silberberg Y 2005 Nonlinear interactions with an ultrahigh flux of broadband entangled photons Phys. Rev. Lett. 94043602

[55] Dayan B, Pe'er A, Friesem A A and Silberberg Y 2004 Two photon absorption and coherent control with broadband down-converted light Phys. Rev. Lett. 9323005

[56] Bessire B, Bernhard C, Feurer T and Stefanov A 2014 Versatile shaper-assisted discretization of energy-time entangled photons New J. Phys. 16033017

[57] Lerch S and Stefanov A 2018 Observing the transition from quantum to classical energy correlations with photon pairs Commun. Phys. 126

[58] Stefanov A 2017 On the role of entanglement in two-photon metrology Quantum Sci. Technol. 2025004

[59] Harris S E 2007 Chirp and compress: toward single-cycle biphotons Phys. Rev. Lett. 98063602

[60] Svozilík J, Peřina J and León-Montiel R D J 2018 Twophoton absorption spectroscopy using intense phase-chirped entangled beams Chem. Phys. 510 54-9

[61] Caves C M 1981 Quantum mechanical noise in an interferometer Phys. Rev. D 231693

[62] Giovannetti V, Lloyd S and Maccone L 2011 Advances in quantum metrology Nat. Photon 5222

[63] Paris M G A 2009 Quantum estimation for quantum technology Int. J. Quantum Inf. 7125
[64] Ragy S, Jarzyna M and Demkowicz-Dobrzański R 2016 Compatibility in multiparameter quantum metrology Phys. Rev. A 94052108

[65] Albarelli F, Friel J F and Datta A 2019 Evaluating the holevo Cramér-Rao bound for multiparameter quantum metrology Phys. Rev. Lett. 123200503

[66] Humphreys P C, Barbieri M, Datta A and Walmsley I A 2013 Quantum enhanced multiple phase estimation Phys. Rev. Lett. 111070403

[67] Polino E, Riva M, Valeri M, Silvestri R, Corrielli G, Crespi A, Spagnolo N, Osellame R and Sciarrino F 2019 Experimental multiphase estimation on a chip Optica 6 288-95

[68] Mikhalychev A B, Bessire B, Karuseichyk I L, Sakovich A A, Unternährer M, Lyakhov D A, Michels D L, Stefanov A and Mogilevtsev D 2019 Efficiently reconstructing compound objects by quantum imaging with higher-order correlation functions Commun. Phys. 2134

[69] Parniak M, Borówka S, Boroszko K, Wasilewski W, Banaszek K and Demkorwicz-Dobrzanski R 2018 Beating the Rayleigh limit using two-photon interference Phys. Rev. Lett. 121250503

[70] Szczykulska M, Baumgratz T and Datta A 2017 Reaching for the quantum limits in the simultaneous estimation of phase and phase diffusion Quantum Sci. Technol. 2 044004

[71] Roccia E, Cimini V, Sbroscia M, Gianani I, Ruggiero L, Mancino L, Genoni M G, Ricci M A and Barbieri M 2018 Multiparameter approach to quantum phase estimation with limited visibility Optica 5 1171-6

[72] Gessner M, Pezzè L and Smerzi A 2018 Sensitivity bounds for multiparameter quantum metrology Phys. Rev. Lett. 121 130503

[73] Ciampini M A, Spagnolo N, Vitelli C, Pezzè L, Smerzi A and Sciarrino F 2016 Quantum-enhanced multiparameter estimation in multiarm interferometers Sci. Rep. 628881

[74] Smith B C 2011 Fundamentals of Fourier Transform Infrared Spectroscopy (Boca Raton, FL: CRC Press)

[75] Zou X Y, Wang L J and Mandel L 1991 Induced coherence and indistinguishability in optical interference Phys. Rev. Lett. 67 318-21

[76] Chekhova M V and Ou Z Y 2016 Nonlinear interferometers in quantum optics Adv. Opt. Photonics 8 104-55

[77] Lemos G B, Borish V, Cole G D, Ramelow S, Lapkiewicz R and Zeilinger A 2014 Quantum imaging with undetected photons Nature 512 409-12

[78] Korystov D Y, Kulik S P and Penin A N 2001 Rozhdestvenski hooks in two-photon parametric light scattering J. Exp. Theor. Phys. Lett. 73 214-8

[79] Kalashnikov D A, Paterova A V, Kulik S P and Krivitsky L A 2016 Infrared spectroscopy with visible light Nat. Photonics 10 98-101

[80] Paterova A V, Lung S, Kalashnikov D A and Krivitsky L A 2017 Nonlinear infrared spectroscopy free from spectral selection Sci. Rep. 742608

[81] Paterova A V, Yang H, An C, Kalashnikov D A and Krivitsky L A 2018 Measurement of infrared optical constants with visible photons New J. Phys. 20043015

[82] Paterova A V, Yang H, An C, Kalashnikov D A and Krivitsky L A 2018 Tunable optical coherence tomography in the infrared range using visible photons Quantum Sci. Technol. 3025008

[83] Paterova A V, Yang H, An C, Kalashnikov D A and Krivitsky L A 2019 Polarization effects in the nonlinear interference of down-converted photons Opt. Express 272589

[84] Freund I 1972 Nonlinear x-ray diffraction. Determination of valence electron charge distributions Chem. Phys. Lett. 12583 
[85] Danino H and Freund I 1981 Parametric down conversion of x rays into the extreme ultraviolet Phys. Rev. Lett. 461127

[86] Tamasaku K and Ishikawa T 2007 Interference between compton scattering and x-ray parametric down-conversion Phys. Rev. Lett. 98244801

[87] Tamasaku K, Sawada K and Ishikawa T 2009 Determining $\mathrm{x}$-ray nonlinear susceptibility of diamond by the optical Fano effect Phys. Rev. Lett. 103254801

[88] Tamasaku K, Sawada K, Nishibori E and Ishikawa T 2011 Visualizing the local optical response to extreme-ultraviolet radiation with a resolution of $\lambda / 380$ Nat. Phys. 7705

[89] Schori A, Bömer C, Borodin D, Collins S P, Detlefs B, Moretti Sala M, Yudovich S and Shwartz S 2017 Parametric down-conversion of x rays into the optical regime Phys. Rev. Lett. 119253902

[90] Borodin D, Levy S and Shwartz S 2017 High energyresolution measurements of $\mathrm{x}$-ray into ultraviolet parametric down-conversion with an x-ray tube source Appl. Phys. Lett. 110131101

[91] Mukamel S 1995 Principles of Nonlinear Spectroscopy (Oxford: Oxford University Press)

[92] Schlawin F, Dorfman K E and Mukamel S 2018 Entangled two-photon absorption spectroscopy Acc. Chem. Res. 512207

[93] Schlawin F, Dorfman K E, Fingerhut B P and Mukamel S 2013 Suppression of population transport and control of exciton distributions by entangled photons Nat. Commun. 41782

[94] Varnavski O, Pinsky B and Goodson T III 2017 Entangled photon excited fluorescence in organic materials: an ultrafast coincidence detector J. Phys. Chem. Lett. 8 388-93

[95] Kalashnikov D A, Pan Z, Kuznetsov A I and Krivitsky L A 2014 Quantum spectroscopy of plasmonic nanostructures Phys. Rev. X 4011049

[96] Raymer M G, Marcus A H, Widom J R and Vitullo D L P 2013 Entangled photon-pair two-dimensional fluorescence spectroscopy (EPP-2DFS) J. Phys. Chem. B 11715559

[97] Shalm L K, Hamel D R, Yan Z, Simon C, Resch K J and Jennewein T 2013 Three-photon energy-time entanglement Nat. Phys. 919

[98] Herrera F, Peropadre B, Pachon L A, Saikin S K and Aspuru-Guzik A 2014 Quantum nonlinear optics with polar J-aggregates in microcavities J. Phys. Chem. Lett. 53708

[99] Zumofen G, Mojarad N M, Sandoghdar V and Agio M 2008 Perfect Reflection of light by an oscillating dipole Phys. Rev. Lett. 101180404

[100] Faez S, Türschmann P, Haakh H, Götzinger S and Sanodghdar V 2014 Coherent interaction of light and single molecules in a dielectric nanoguide Phys. Rev. Lett. 113 213601

[101] Türschmann P, Rotenberg N, Renger J, Harder I, Lohse O, Utikal T, Götzinger S and Sandoghdar V 2017 Chip-based all-optical control of single molecules coherently coupled to a nanoguide Nano Lett. 74941

[102] Haakh H, Faez S and Sandoghdar V 2016 A polaritonic normal-mode splitting and light localization in a onedimensional nanoguide Phys. Rev. A 94053840

[103] Asenjo-Garcia A, Hood J D, Chang D E and Kimble H J 2017 Phys. Rev. A 95033818

[104] Sipahigil A et al 2016 An integrated diamond nanophotonics platform for quantum-optical networks Science $\mathbf{3 5 4} 847$

[105] Wang D, Kelkar H, Cano D-M, Rattenbacher D, Shkarin A, Utikal T, Götzinger S and Sandoghdar V 2019 Turning a molecule into a coherent two-level quantum system Nat. Phys. 15483

[106] Rezus Y L A, Walt S G, Lettow R, Renn A, Zumofen G, Götzinger S and Sandoghdar V 2012 Single-photon spectroscopy of a single molecule Phys. Rev. Lett. 108093601
[107] Feynman R 1960 There's plenty of room at the bottom Caltech Eng. Sci. 2322

[108] Raymer M G, Marcus A H, Widom J R and Vitullo D L P 2013 Entangled photon-pair two dimensional fluorescence spectroscopy (EPP-2DFS) J. Phys. Chem. B 117 15559-75

[109] Tekavec P F, Lott G A and Marcus A H 2007 Fluorescencedetected two-dimensional electronic coherence spectroscopy by acousto-optic phase modulation J. Chem. Phys. 127 214307

[110] Fuller F D and Ogilvie J P 2015 Experimental implementations of two-dimensional Fourier transform electronic spectroscopy Annu. Rev. Phys. Chem. 66 667-90

[111] Turner D B, Arpin P C, McClure S D, Ulness D J and Scholes G D 2013 Coherent multidimensional optical spectra measured using incoherent light Nat. Commun. 4 2298-307

[112] Hong C K, Ou Z Y and Mandel L 1987 Measurement of subpicosecond time intervals between two photons by interference Phys. Rev. Lett. 59 2044-8

[113] Grice W and Walmsley I 1997 Spectral information and distinguishability in type-II down-conversion with a broadband pump Phys. Rev. A 56 1627-34

[114] Franson J D 1989 Bell inequalities for position and time Phys. Rev. Lett. $622205-8$

[115] Kaltenbaek R, Lavoie J and Resch K J 2009 Classical analogues of two-photon quantum interference Phys. Rev. Lett. 102243601

[116] Perdomo A, Widom J R, Lott G A, Aspuru-Guzik A and Marcus A H 2012 Conformation and electronic population transfer in membrane supported self-assembled porphyrin dimers by two-dimensional fluorescence spectroscopy J. Phys. Chem. B 116 10757-70

[117] Kringle L, Sawaya N P D, Widom J, Adams C, Raymer M G, Aspuru-Guzik A and Marcus A H 2018 Temperaturedependent conformations of exciton-coupled Cy3 dimers in double-stranded DNA J. Chem. Phys. 148085101

[118] Schlawin F and Buchleitner A 2017 Theory of coherent control with quantum light New J. Phys. 19013009

[119] Davis A O C, Thiel V and Smith B J 2019 Measuring the quantum state of a photon pair entangled in frequency and time (arXiv:1809.03727)

[120] MacLean J-P W, Donohue J M and Resch K J 2018 Direct characterization of ultrafast energy-time entangled photon pairs Phys. Rev. Lett. 120053601

[121] Lee D I and Goodson T 2006 Entangled photon absorption in an organic porphyrin dendrimer J. Phys. Chem. B $\mathbf{1 1 0}$ 25582-5

[122] Harpham M R, Süzer Ö, Ma C-Q, Bäuerle P and Goodson T III 2009 Thiophene dendrimers as entangled photon sensor materials J. Am. Chem. Soc. 131 973-9

[123] Upton L, Harpham M, Suzer O, Richter M, Mukamel S and Goodson T 2013 Optically excited entangled states in organic molecules illuminate the dark J. Phys. Chem. Lett. 4 2046-52

[124] Burdick R K, Varnavski O, Molina A, Upton L, Zimmerman P and Goodson T III 2018 Predicting and controlling entangled two-photon absorption in diatomic molecules J. Phys. Chem. A 122 8198-212

[125] Guzman A R, Harpham M R, Süzer Ö, Haley M M and Goodson T G III 2010 Spatial control of entangled twophoton absorption with organic chromophores J. Am. Chem. Soc. 132 7840-1

[126] Villabona-Monsalve J P, Varnavski O, Bruce A, Palfey B A and Goodson III T 2018 Two-photon excitation of flavins and flavoproteins with classical and quantum light J. Am. Chem. Soc. 140 14562-6

[127] Eshun A, Cai Z, Awies M, Yu L and Goodson T III 2018 Investigations of thienoacene molecules for classical and 
entangled two-photon absorption J. Phys. Chem. A 122 8167-82

[128] Dorfman K E, Schlawin F and Mukamel S 2016 Nonlinear optical signals and spectroscopy with quantum light Rev. Mod. Phys. 88045008

[129] Raymer M G, Marcus A H, Julia R, Widom J R, Dashiell L P and Vitullo D L P 2013 Entangled photon-pair two-dimensional fluorescence spectroscopy (EPP-2DFS) J. Phys. Chem. B 117 15559-75

[130] Allen L, Beijersbergen M W, Spreeuw R J C and Woerdman J P 1992 Orbital angular momentum of light and transformation of Laguerre-Gaussian mode Phys. Rev. A 45 8185-9

[131] Franke-Arnold S, Allen L and Padgett M 2008 Advances in optical angular momentum Laser Photonics Rev. 2 299-313

[132] Brullot W, Vanbel M K, Swusten T and Verbiest T 2016 Resolving enantionmers using the optical angular momentum of twisted light Sci. Adv. 2 e1501349

[133] Tischler N, Krenn M, Fickler R, Vidal X, Zeilinger A and Molina-Terriza G 2016 Quantum optical rotatory dispersion Sci. Adv. 2 e1601306

[134] Zhou Z Y, Li Y, Ding D S, Zhang W, Shi S and Shi B S 2015 Classical to quantum optical network link for orbital angular momentum-carrying light Opt. Express 23 18435-44

[135] Ding D S, Zhang W, Zhou Z Y, Shi S, Xiang G Y, Wang X S, Jiang Y K, Shi B S and Guo G C 2015 Quantum storage of orbital angular momentum entanglement in an atomic ensemble Phys. Rev. Lett. 114050502

[136] Erhard M, Fickler R, Krenn M and Zeilinger A 2018 Twisted photons: new quantum perspectives in high dimensions Light: Sci. Appl. 7 e17146

[137] Zhou Z Y, Liu S L, Li Y, Ding D S, Zhang W, Shi S, Dong M X, Shi B S and Shi B S 2016 Orbital angular momentum entanglement frequency transducer Phys. Rev. Lett. 117103601

[138] Zhou Z Y, Liu S K, Liu S L, Li Y, Yang C, Xu Z H, Guo G C and Shi B S 2018 Revealing the behavior of photons in a birefringent interferometer Phys. Rev. Lett. 120 263601

[139] Asban S, Dorfman K E and Mukamel S 2019 Quantum phase-sensitive diffraction and imaging using entangled photons Proc. Natl Acad. Sci.

[140] Dorfman K E, Asban S, Ye L, Rouxel J R, Cho D and Mukamel S 2019 Monitoring spontaneous charge-density fluctuations by single-molecule di raction of quantum light J. Phys. Chem. Lett. $10768-73$

[141] Straupe S S, Ivanov D P, Kalinkin A A, Bobrov I B and Kulik S P 2011 Angular Schmidt modes in spontaneous parametric down-conversion Phys. Rev. A 8360302

[142] Brida G, Genovese M and Ruo Berchera I 2010 Experimental realization of sub-shot-noise quantum imaging Nat. Photonics 4 227-30

[143] Mukamel S 1995 Principles of Nonlinear Optical Spectroscopy (Oxford Series in Optical and Imaging Sciences) (Oxford: Oxford University Press)

[144] Vartanyants I A and Singer A 2018 Coherence Properties of Third-Generation Synchrotron Sources and Free Electron Lasers (Berlin: Springer) pp 1-38

[145] Bennett K, Biggs J D, Zhang Y, Dorfman K E, Mukamel S, Bennett K, Biggs J D, Zhang Y and Dorfman K E 2014 Time-, frequency-, and wavevector-resolved X-ray diffraction from single molecules J. Chem. Phys. 140 204311

[146] Scully M O and Druhl K 1982 Quantum eraser: a proposed photon correlation experiment concerning observation and 'delayed choice' in quantum mechanics Phys. Rev. A 25 2208-13

[147] Scully M O, Englert B-G and Walther H 1991 Quantum optical tests of complementarity Nature 351 111-6
[148] Scully M O 2005 Improving quantum microscopy via Raman photon pairs Concept Phys. 2 261-72

[149] Muthukrishnan A, Scully M O and Suhail Zubairy M 2004 Quantum microscopy using photon correlations J. Opt. B 6 S575-82

[150] Kim Y H, Yu R, Kulik S P, Shih Y H and Scully M O 2000 Delayed 'choice' quantum eraser Phys. Rev. Lett. 84 1-5

[151] Rathe U and Scully M O 1995 Theoretical basis for a new subnatural spectroscopy via correlation interferometry Lett. Math. Phys. 34 297-307

[152] Scully M O and Zubairy S 1997 Quantum Optics (Cambridge: Cambridge University Press)

[153] Thiel C, Bastin T, Martin J, Solano E, von Zanthier J and Agarwal G S 2007 Quantum Imaging with incoherent photons Phys. Rev. Lett. $99133603-6$

[154] D'Angelo M, Chekhova M and Shih Y 2001 Two-photon diffraction and quantum lithography Phys. Rev. Lett. 87 013602-5

[155] Brown R H and Twiss R Q 1956 A test of a new type of stellar interferometer on sirius Nature 1781046

[156] Glauber R J 1963 The quantum theory of optical coherence Phys. Rev. 1302529

[157] Vartanyants I A et al 2011 Coherence properties of individual femtosecond pulses of an x-ray free-electron laser Phys. Rev. Lett. 107144801

[158] Singer A et al 2012 Spatial and temporal coherence properties of single free-electron laser pulses Opt. Express 20 17480-95

[159] Singer A et al 2013 Hanbury Brown-Twiss interferometry at a free-electron laser Phys. Rev. Lett. 111034802

Singer A 2016 Phys. Rev. Lett. 117239903

[160] Gorobtsov O Y et al 2017 Statistical properties of a freeelectron laser revealed by the Hanbury Brown and Twiss interferometry Phys. Rev. A 95023843

[161] Gorobtsov O Y et al 2018 Diffraction based Hanbury Brown and Twiss interferometry at a hard $\mathrm{x}$-ray free-electron laser Sci. Rep. 82219

[162] Gorobtsov O Y et al 2018 Seeded x-ray free-electron laser generating radiation with laser statistical properties Nat. Commun. 94498

[163] Agarwal G 2013 Quantum Optics (Cambridge: Cambridge University Press)

[164] Schneider R et al 2018 Quantum imaging with incoherently scattered light from a free-electron laser Nat. Phys. 14 126-9

[165] Pittman T B, Shih Y H, Strekalov D V and Sergienko A V 1995 Phys. Rev. A 52 R3429

[166] Kim Y Y et al 2020 Ghost imaging at an XUV free-electron laser Phys. Rev. A 101013820

[167] Aspect A, Roger G, Reynaud S, Dalibard J and Cohen-Tannoudji C 1980 Time correlations between two sidebands of the resonance fluorescence triplet Phys. Rev. Lett. 45617

[168] Apanasevich P A and Kilin S Ja 1979 Photon bunching and antibunching in resonance fluorescence J. Phys. B: At. Mol. Opt. Phys. 12 L83

[169] Eberly J and Wódkiewicz K 1977 The time-dependent physical spectrum of light J. Opt. Soc. Am. 671252

[170] Knöll L, Vogel W and Welsch D-G 1990 Spectral properties of light in quantum optics Phys. Rev. A 42503

[171] Nienhuis G 1993 Spectral correlations in resonance fluorescence Phys. Rev. A 47510

[172] del Valle E, Gonzalez-Tudela A, Laussy F P, Tejedor C and Hartmann M J 2012 Theory of frequency-filtered and timeresolved N-photon correlations Phys. Rev. Lett. 109183601

[173] Shatokhin V N and Kilin S Y 2016 Correlation functions in resonance fluorescence with spectral resolution: signalprocessing approach Phys. Rev. A 94033835

[174] López Carreño J C, del Valle E and Laussy F P 2018 Frequency-resolved Monte Carlo Sci. Rep. 86975 
[175] Gonzalez-Tudela A, Laussy F P, Tejedor C,

Hartmann M J and del Valle E 2013 Two-photon spectra of quantum emitters New J. Phys. 15033036

[176] Sánchez Muñoz C, del Valle E, Tejedor C and Laussy F P 2014 Violation of classical inequalities by frequency filtering Phys. Rev. A 90052111

[177] Gonzalez-Tudela A, del Valle E and Laussy F P 2015 Optimization of photon correlations by frequency filtering Phys. Rev. A 91043807

[178] Peiris M, Petrak B, Konthasinghe K, Yu Y, Niu Z C and Muller A 2015 Two-color photon correlations of the light scattered by a quantum dot Phys. Rev. B 91195125

[179] Silva B, Sánchez-Muñoz C, Ballarini D, González-Tudela A, Gigli G, West K W, Pfeiffer L, del Valle E, Sanvitto D and Laussy F P 2016 The colored Hanbury Brown-Twiss effect Sci. Rep. 637980
[180] López Carreño J C, del Valle E and Laussy F P 2017 Photon correlations from the Mollow triplet Laser Photonics Rev. 11 1700090

[181] Sánchez-Muñoz C, del Valle E, González-Tudela A, Lichtmannecker S, Müller K, Kaniber M, Tejedor C, Finley J J and Laussy F P 2014 Emitters of $N$-photon bundles Nat. Photonics 8550

[182] López Carreño J C, Sánchez Muñoz C, Sanvitto D, del Valle E and Laussy F P 2015 Exciting polaritons with quantum light Phys. Rev. Lett. 115196402

[183] Sánchez-Muñoz C, Laussy F P, del Valle E, Tejedor C and González-Tudela A 2018 Filtering multiphoton emission from state-of-the-art cavity quantum electrodynamics Optica $\mathbf{5} 14$

[184] Saleh B E A, Jost B M, Fei H-B and Teich M C 1998 Entangled-photon virtual-state spectroscopy Phys. Rev. Lett. 80 3483-6 